\title{
Biodiversity, Species Interactions and Ecological Networks in a Fragmented World
}

Melanie Hagen*, W. Daniel Kissling*, Claus Rasmussen*, Marcus A.M. De Aguiar ${ }^{\dagger}$, Lee E. Brown*, Daniel W. Carstensen*, Isabel Alves-Dos-Santos ${ }^{\S}$, Yoko L. Dupont*, Francois K. Edwards", Julieta Geninill, Paulo R. Guimarães Jr. ${ }^{\S}$, Gareth B. Jenkins", Pedro Jordano**, Christopher N. Kaiser-Bunbury*, Mark E. Ledger ${ }^{\dagger \dagger}$, Kate P. Maia ${ }^{\S}$, Flavia M. Darcie Marquitti ${ }^{\S}$, Órla Mclaughlin $\$, \S \S$, L. Patricia C. Morellato", Eoin J. O'Gorman", Kristian Trøjelsgaard*, Jason M. Tylianakis ${ }^{\top \uparrow}$, Mariana Morais Vidal ${ }^{\S}$, Guy Woodward", Jens M. Olesen*,1

*Department of Bioscience, Aarhus University, Aarhus, Denmark

†Instituto de Física Gleb Wataghin, Universidade Estadual de Campinas, Campinas, São Paulo, Brazil

${ }^{*}$ School of Geography, University of Leeds, Leeds, United Kingdom

${ }^{\S}$ Departamento de Ecologia, Instituto de Biociências, Universidade de São Paulo, São Paulo, São Paulo, Brazil 'Centre for Ecology and Hydrology, Wallingford, United Kingdom

"Departamento de Botânica, Laboratório de Fenologia, UNESP Univ Estadual Paulista, Rio Claro, São Paulo, Brazil ${ }^{\#}$ School of Biological and Chemical Sciences, Queen Mary University of London, London, United Kingdom **Integrative Ecology Group, Estación Biológica de Doñana, CSIC, Sevilla, Spain

${ }^{\dagger}$ School of Geography, Earth and Environmental Sciences, University of Birmingham, Edgbaston, Birmingham, United Kingdom

林Environmental Research Institute, University College Cork, Cork, Ireland

${ }^{\S}$ School of Biological, Earth, and Environmental Sciences, University College Cork, Cork, Ireland

"School of Biological Sciences, University of Canterbury, Christchurch, New Zealand

${ }^{1}$ Corresponding author: e-mail address: jens.olesen@biology.au.dk

\section{Contents}

1. Introduction 91

2. Networks 94

2.1 Ecological networks 94

$\begin{array}{ll}2.2 \text { Spatial networks } & 100\end{array}$

$\begin{array}{ll}2.3 \text { Combining spatial and ecological networks } & 101\end{array}$

3. Habitat Fragmentation 103

3.1 General introduction 103

$\begin{array}{lll}3.2 & \text { Fragment characteristics } & 107\end{array}$

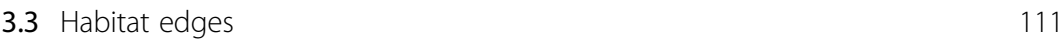

$\begin{array}{ll}3.4 \text { Matrix } & 114\end{array}$

3.5 Spatial and temporal turnover of species and individuals 117

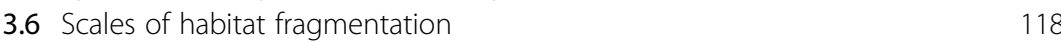


4. Habitat Fragmentation and Species Traits 121

4.1 Plant traits $\quad 122$

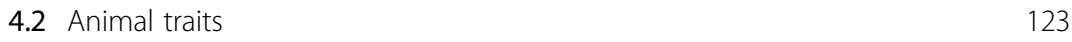

$\begin{array}{lll}4.3 & \text { Species trait combinations } & 126\end{array}$

5. Habitat Fragmentation and Biotic Interactions 129

$\begin{array}{ll}5.1 \text { Mutualistic plant-pollinator interactions } & 129\end{array}$

$\begin{array}{ll}5.2 \text { Mutualistic plant-frugivore interactions } & 131\end{array}$

$\begin{array}{ll}\text { 5.3 Mutualistic plant-ant interactions } & 132\end{array}$

$\begin{array}{ll}\text { 5.4 Antagonistic interactions within food webs } & 134\end{array}$

$\begin{array}{ll}\text { 5.5 Antagonistic host-parasitoid interactions } & 135\end{array}$

5.6 Summary of fragmentation effects on mutualistic and antagonistic interactions

$\begin{array}{ll}\text { 6.1 General introduction } & 138 \\ 6.2 \text { Mutualistic plant-pollinator networks } & 138\end{array}$

$\begin{array}{ll}\text { 6.3 Mutualistic plant-frugivore networks } & 150\end{array}$

$\begin{array}{ll}\text { 6.4 Mutualistic plant-ant networks } & 153\end{array}$

$\begin{array}{ll}\text { 6.5 Antagonistic food webs } & 154\end{array}$

$\begin{array}{ll}6.6 \text { Antagonistic host-parasitoid networks } & 160\end{array}$

6.7 General effects of habitat fragmentation on network properties 162

7. Habitat Fragmentation in a Meta-Network Context 164

$\begin{array}{lll}7.1 & \text { Meta-networks and dispersal } & 166\end{array}$

$\begin{array}{lll}7.2 & \text { Meta-networks and extinction } & 166\end{array}$

$\begin{array}{ll}\text { 7.3 Meta-networks and colonisation } & 167\end{array}$

8. Effects of Habitat Fragmentation on the Coevolutionary Dynamics of Networks 169

$\begin{array}{ll}\text { 8.1 The geographic mosaic theory of coevolution } & 169\end{array}$

8.2 Habitat fragmentation and its effects on basic components of GMTC 170

8.3 Habitat fragmentation and selection mosaics in ecological networks 171

9. Applications in Conservation and Agriculture 172

10. Conclusions 175

$\begin{array}{ll}\text { Acknowledgements } & 177\end{array}$

$\begin{array}{ll}\text { Appendix } & 177\end{array}$

$\begin{array}{ll}\text { References } & 181\end{array}$

\section{Abstract}

Biodiversity is organised into complex ecological networks of interacting species in local ecosystems, but our knowledge about the effects of habitat fragmentation on such systems remains limited. We consider the effects of this key driver of both local and global change on both mutualistic and antagonistic systems at different levels of biological organisation and spatiotemporal scales.

There is a complex interplay of patterns and processes related to the variation and influence of spatial, temporal and biotic drivers in ecological networks. Species traits (e.g. body size, dispersal ability) play an important role in determining how networks respond to fragment size and isolation, edge shape and permeability, and the quality of 
the surrounding landscape matrix. Furthermore, the perception of spatial scale (e.g. environmental grain) and temporal effects (time lags, extinction debts) can differ markedly among species, network modules and trophic levels, highlighting the need to develop a more integrated perspective that considers not just nodes, but the structural role and strength of species interactions (e.g. as hubs, spatial couplers and determinants of connectance, nestedness and modularity) in response to habitat fragmentation.

Many challenges remain for improving our understanding: the likely importance of specialisation, functional redundancy and trait matching has been largely overlooked. The potentially critical effects of apex consumers, abundant species and supergeneralists on network changes and evolutionary dynamics also need to be addressed in future research. Ultimately, spatial and ecological networks need to be combined to explore the effects of dispersal, colonisation, extinction and habitat fragmentation on network structure and coevolutionary dynamics. Finally, we need to embed network approaches more explicitly within applied ecology in general, because they offer great potential for improving on the current species-based or habitat-centric approaches to our management and conservation of biodiversity in the face of environmental change.

\section{INTRODUCTION}

The planet's ecosystems are losing biodiversity at an accelerating rate (Dyer et al., 2010; Fahrig, 2003; Gonzalez et al., 2011; Millennium Ecosystem Assessment, 2005) due to land-use change, deforestation, agricultural intensification, pollution, urbanisation, climate change and habitat fragmentation (Albrecht et al., 2007; Hanski, 2005; Ledger et al., 2012; Meerhoff et al., 2012; Mintenbeck et al., 2012; Tilman et al., 2001). The latter in particular could severely disrupt ecological networks and the goods and services they provide (e.g. pollination in mutualistic webs or biological control in food webs) as it is a rapidly growing phenomenon throughout the world, yet its impacts on the higher multispecies levels of organisation are still poorly understood.

A major challenge for predicting the consequences of changes on biodiversity is to understand the complexity of natural systems and the steps needed to conserve them in a rapidly changing world. Biodiversity is organised at local scales into complex networks of interacting species, which provide the ecosystem processes that ultimately underpin the goods and services of value to human societies (Rossberg, 2012). These links (italicised terms, see Glossary) among interacting species are often ignored in the context of global change even though they will disappear from local 
communities as a precursor to local (and ultimately global) extinctions (Albrecht et al., 2007; Fortuna and Bascompte, 2006; Sabatino et al., 2010; Tylianakis et al., 2007; Woodward et al., 2010a). Understanding the causes and consequences of the loss of species interactions therefore promises to provide critical new insights into ecological responses to perturbations (Mulder et al., 2012; Tylianakis et al., 2010).

The interplay between the abiotic environment and biotic complexity over space and time makes natural ecosystems seemingly difficult to understand. One simplifying approach is to study interactions among multiple species in the framework of ecological networks (e.g. Fortuna and Bascompte, 2008). These include both mutualistic (e.g. pollination, seed dispersal networks) and antagonistic (e.g. food webs, host-parasitoid networks) interactions, which could respond differently to disturbances, such as fragmentation, which in turn determines their stability in terms of resilience, resistance and robustness (Ings et al., 2009; Layer et al., 2010, 2011; Woodward et al., 2010a).

Landscape changes may be caused by physical processes, biotic drivers such as ecological engineers, and/or anthropogenic influences. Species will reshuffle their population sizes and some links between species might be rewired or break apart entirely (Tscharntke et al., 2005). Any seemingly restricted spatiotemporal disturbance may ripple throughout the network of interacting species, causing further (i.e. secondary) species and link perturbations. New data analytical tools, such as network analysis, now form an essential ingredient in the study of complex systems, with clear implications for biodiversity research (Heleno et al., 2009; Kremen and Hall, 2005; Tylianakis et al., 2008).

Habitat fragmentation is almost ubiquitous in both natural and humanmodified landscapes (Fig. 1), with consequences for biodiversity and species interactions (Fahrig, 2003; Laurance et al., 2011; Tylianakis et al., 2007), which in turn has implications for the entire ecological network. It reduces habitat area and species connectivity, and the sizes and isolation of remaining fragments are particularly critical to the long-term conservation of biodiversity. Connectivity among fragments, the characteristics of the matrix, the availability of corridors for movement between fragments, and the permeability and structure of habitat edges are all important in this context and affect the structure, persistence and strength of species interactions (Fortuna and Bascompte, 2006). Certain species traits (e.g. body size, dispersal ability, degree of specialisation or trophic rank) are likely to be particularly crucial for assessing the higher-level consequences 


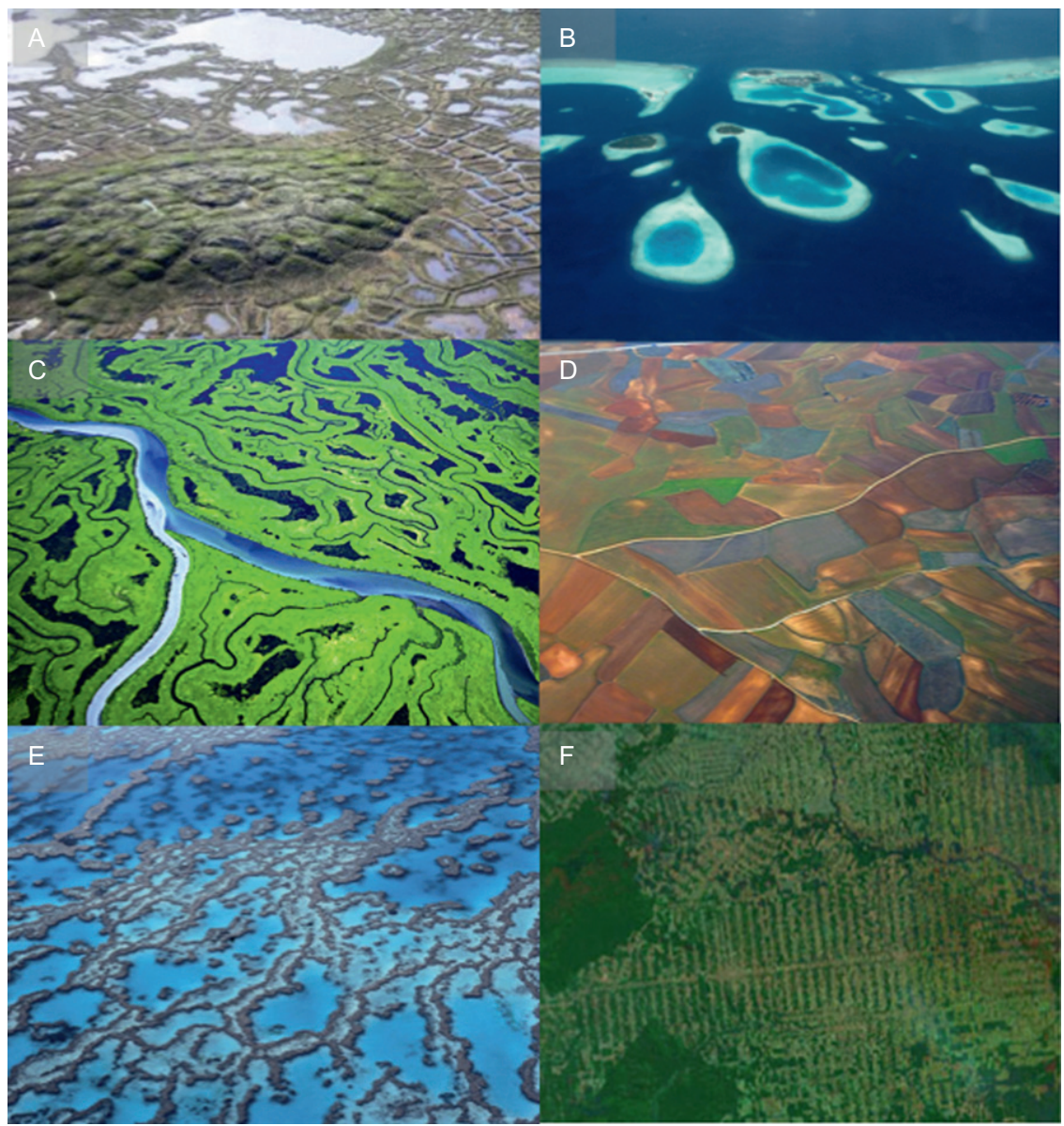

Figure 1 The ubiquity of fragmentation. Selected examples of common naturally and artificially fragmented habitats from terrestrial and aquatic ecosystems, with hard (aquatic-terrestrial) versus soft (aquatic-aquatic, terrestrial-terrestrial) boundaries. From top left to bottom right are (A) pingos in the arctic; (B) tropical atoll islands; (C) temperate river network and associated off-river habitats; (D) agricultural landscape in Spain; (E) a portion of the Great Barrier Reef and (F) forest clearance in Amazonia.

of habitat fragmentation (Ewers and Didham, 2006), so functional attributes may be just as important as taxonomic diversity in this context. The invasion of functionally similar species, for example, may homogenise ecological processes (McKinney and Lockwood, 1999; Olden et al., 2004). Species at higher trophic levels, or with particular traits, that connect 
different fragments or network modules, may act as important spatial couplers or network stabilisers, essentially operating as network-level keystones.

Both the physical and biological worlds can be seen as networks (Gonzalez et al., 2011): a (spatial) landscape network of habitat fragments that provides the underlying matrix and habitat connectivity, and an ecological species interaction network, driven by ecological and evolutionary processes. Interactions between such different kinds of networks occur, but to date such multiple interdependent networks have mainly been studied outside ecology (Buldyrev et al., 2010) and the consequences of habitat fragmentation on these (often interdependent) biological-physical systems remain largely unexplored.

Here, we synthesise current knowledge about the consequences of habitat fragmentation on different types of biodiversity within ecological networks. We begin by introducing the major characteristics and types of ecological and spatial networks. We then review the spatial and temporal settings of habitat fragmentation, including fragment characteristics, habitat edges, matrix quality and permeability, spatial and temporal turnover of species and individuals, and different scales of fragmentation. We illustrate how habitat fragmentation effects depend on species traits, paying particular attention to both mutualistic (plant-pollinator, plant-frugivore, plant-ant) and antagonistic (host-parasitoid, food web) interactions, and we synthesise current knowledge on likely consequences for ecological networks and make suggestions about future research directions. Finally, we summarise possible applications for conservation, agriculture and applied ecology in general. Throughout the paper, we consider different kinds of interactions and networks across a range of spatiotemporal scales.

\section{NETWORKS}

\subsection{Ecological networks}

Networks contain nodes and their links: in ecology, nodes may be individuals, species populations, species, guilds, functional groups (e.g. body-size groups), entire communities, or even entire networks, and interactions can take many forms (e.g. plant-pollinator, plant-frugivore and predator-prey associations (Fig. 2)).

Links in an ecological network are defined in an interaction matrix. The coarsest measure of link strength is simply the occurrence (presence/absence 

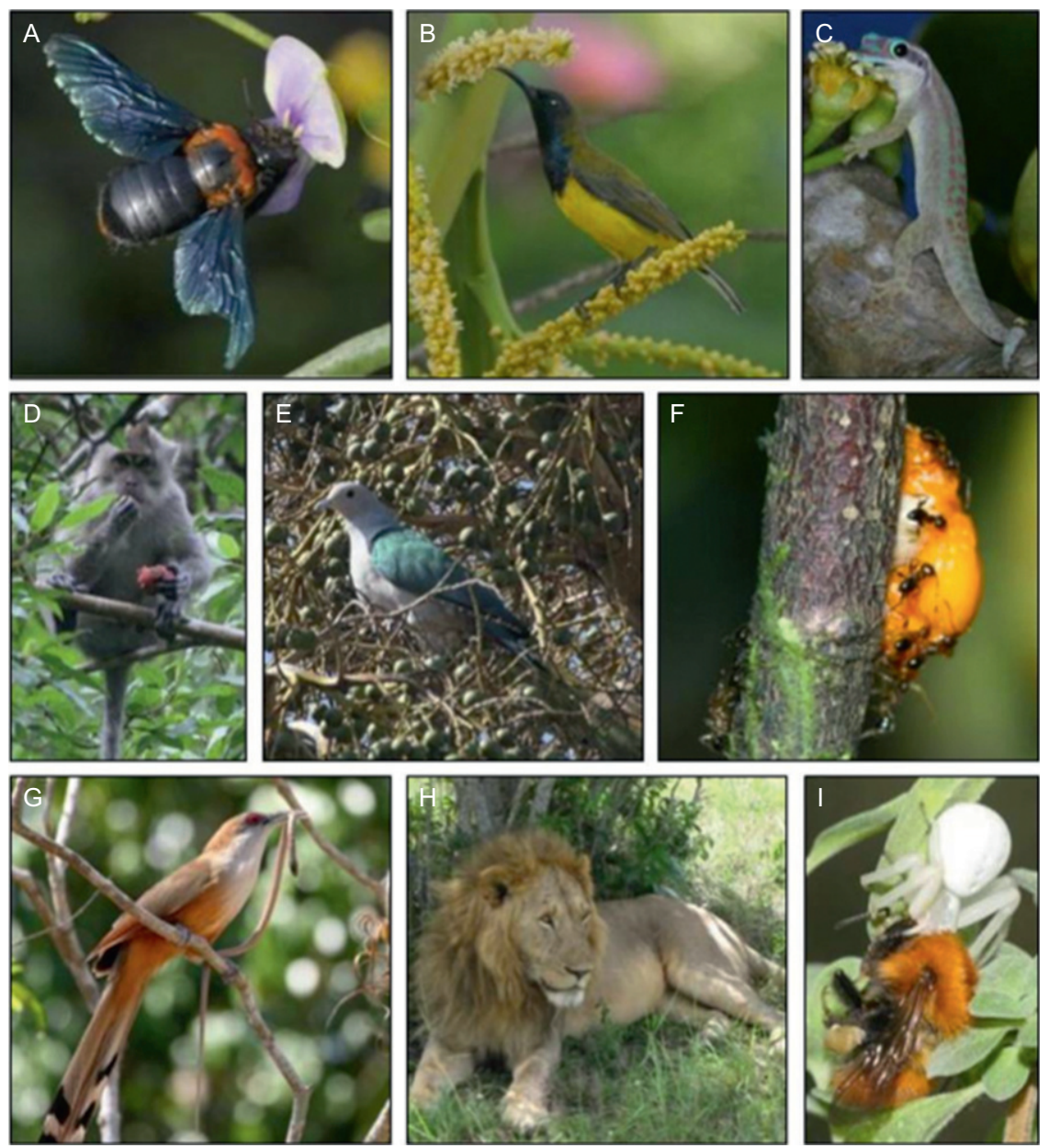

Figure 2 Examples of biotic interactions. (A) Carpenter bee (Xylocopa flavorufa) pollinating cowpea (Vicia unguiculata) in Western Kenya (photo: M. Hagen). (B) Sunbird (Cinnyris jugularis) pollinating palm inflorescences in Flores, Indonesia (photo: J. M. Olesen). (C) Day Gecko (Phelsuma ornata) pollinating Gastonia mauritiana in Mauritius (photo: C. Kaiser-Bunbury). (D) Long-tailed Macaque (Macaca fascicularis) consuming figs on Lombok, Indonesia (photo: J. M. Olesen). (E) Green Imperial Pigeon (Ducula aenea) consuming fruits of a palm (Corypha taliera) in Komodo, Indonesia (photo: J. M. Olesen). (F) Seed dispersal of Casearia coriacea by ants in Le Pétrin, Mauritius (photo: C. Kaiser-Bunbury). (G) Great Lizard Cuckoo (Coccyzus merlini) predating a snake in Cuba (photo: J. M. Olesen). (H) African lion (Panthera leo) 'resting' after a biotic interaction in Masai Mara, Kenya (photo: W. D. Kissling). (I) Crab spider predating a bumblebee (Bombus cf. pascuorum) in Liguria, Northern Italy (photo: C. KaiserBunbury). 
data), within qualitative networks, although it can be measured in many ways (Berlow et al., 2004). For instance, for a plant-pollinator network, the links may represent the number of visitors to a plant, number of visits, number of pollen grains transferred to the stigma or number of pollen grains siring seeds, seedlings or reproductive individuals. For food webs, numerous measures and definitions have been described (see review by Berlow et al., 2004), whereas in mutualistic networks the interaction frequency is the norm (Vázquez et al., 2005). Both qualitative and quantitative interaction parameters allow not only the description of local community-level interactions, but also the modelling of multispecies interactions across larger scales (Kissling et al., 2012a).

Mutualistic and antagonistic networks represent the two main groups encountered in the ecological literature, and each has its own historical tradition (Olesen et al., 2012). Thus, antagonistic networks include 'traditional food webs' (typically larger consumers kill and eat many individual prey; e.g. Jacob et al., 2011; Layer et al., 2010, 2011; McLaughlin et al., 2010; O'Gorman et al., 2010), host-parasitoid networks (e.g. Henri and van Veen, 2011; Tylianakis et al., 2007), as well as less-familiar host-parasite or pathogen networks (e.g. Lafferty et al., 2008). Mutualistic networks include plant-flower visitor/pollinator (e.g. Memmott, 1999) and plant-frugivore/seed disperser networks (e.g. Donatti et al., 2011; Schleuning et al., 2011a), with less familiar forms including plant-ant networks (Guimarães et al., 2007) and host-symbiont interactions (e.g. gut microbiomes; Purdy et al., 2010). These categories are not exhaustive, but they represent main foci of current ecological network research (Ings et al., 2009). No doubt new forms of networks will appear as this rapidly growing research field expands its horizons further: for instance, interspecific competition within trophic levels has been largely ignored to date, except in the context of trophic niche partitioning within food webs, but such networks may become important, especially in the context of habitat fragmentation, where space rather than food may be limiting.

Food webs are traditionally divided into aquatic (freshwater and marine) and terrestrial (aboveground and belowground) systems, although some of the oldest food web studies included several habitats (e.g. Pimm and Lawton, 1980). These early ideas are now being revisited increasingly, with a focus upon 'spatial couplers', such as allochthonous inputs at the base of the food web, migratory top predators that link different local webs or species that have both an aquatic and terrestrial life history (Jonsson et al., 2005; 
Layer et al., 2010; McCann et al., 2005a,b; O'Gorman and Emmerson, 2010; Woodward et al., 2005).

Mutualistic and antagonistic webs are inherently difficult to compare directly (e.g. in their responses to fragmentation) because they differ in their structure, dynamics and link type. The former are bipartite or bimodal, that is, consisting of two interacting sets of taxa, whereas the latter are multi-modal, that is, containing multiple trophic levels (e.g. producer-herbivore-predator). One way to approach this might be to slice food webs up according to pairs of interacting trophic levels into a series of bimodal networks, that is, plant-herbivore, herbivore-predator and so on. Alternatively, mutualistic networks, such as plant-pollinator networks, could be merged with other $b i$ modal networks, for example, those of plant-herbivore or plant-fungi networks, to create networks of several interacting groups (see Fontaine et al., 2011; L. Kromann-Gallop, personal communication). Until such an analysis is made, it remains difficult to compare the properties of different kinds of networks directly (but see Olesen et al., 2006), although such comparisons are theoretically possible (Thebault and Fontaine, 2010), and we therefore address both types as separate cases throughout the paper.

\subsubsection{Properties of mutualistic and antagonistic networks}

Common measures of network structure include species and link numbers, connectance, and linkage level distribution, many of which are important because they make implicit connections between network complexity, stability and resource partitioning in ecology (Berlow et al., 2009; Elton, 1927; MacArthur, 1955; May, 1972, 1973; McCann et al., 1998; Warren, 1996; Williams and Martinez, 2000). These measures and their significance in networks have been discussed extensively elsewhere (Berlow et al., 2004; Ings et al., 2009; Olesen et al., 2010b), so we will not cover them in detail here. Instead, we provide a brief overview of the main concepts, with a specific focus on habitat fragmentation.

Networks also display recognisable substructural patterns, often in a fractal-like manner, such that they may contain repeating motifs, modules or compartments within the wider web (e.g. Olesen et al., 2007; Stouffer and Bascompte, 2010). For example, food webs can be decomposed into food chains, tritrophic chains and ultimately their pairwise individual feeding links, each of which may display its own response to habitat fragmentation (Woodward et al., 2012). These have received less attention than the whole-network measures of complexity (e.g. connectance), but in recent years considerable advances have been made, especially in the 
study of mutualistic webs. Substructures could be especially important in the context of habitat fragmentation, as they may represent some form of 'network fragmentation' related to spatial compartmentalisation. For instance, connector species that link modules might be species with large space requirements or long dispersal distances, that join otherwise spatially distinct subwebs. The same principles may apply through time: for instance, top predators move not only over wide distances but also tend to be relatively long-lived, linking seasonally or spatiotemporally fragmented subwebs together (Woodward and Hildrew, 2002a).

The two most common forms of network (sub)structure, nestedness and modularity, have been studied intensively (Bascompte et al., 2003; Lewinsohn et al., 2006; Olesen et al., 2007; Pimm, 1984). In a nested network, the links of specialist species are well-defined subsets of the links of generalists (Bascompte et al., 2003). Modularity describes subsets of species (modules) that are internally highly connected, but poorly connected to other such subsets of species (Olesen et al., 2007). Nestedness and modularity have often been regarded as mutually exclusive (Lewinsohn et al., 2005), but this is not necessarily true (Fortuna et al., 2010; Olesen et al., 2007). Link patterns in bimodal networks vary with presence of links and the frequency or intimacy of interactions between partners (Olesen et al., 2008). If link presence and intimacy are short and weak, the network may become nested and modular, such as in pollination and frugivory/seed dispersal networks, but if prolonged and tight, nestedness may be lost although modularity might be retained, such as in host-parasitoid and plant-ant domatia networks. Generalists and common species may be lost or 'forced' over evolutionary time towards being more specialised and rare. Interaction 'intruders' may also break into the latter networks, making them more nested. Such species are generalists and can also act as spatial couplers in otherwise fragmented networks, as seen in plant-ant domatia networks (Olesen et al., 2002).

\subsubsection{Body size as a driver of ecological network structure}

Body size is an important driver of structure and dynamics in many food webs (Arim et al., 2011; Melián et al., 2011; Nakazawa et al., 2011), especially in aquatic ecosystems (Jacob et al., 2011; Woodward et al., 2005), and can give rise to substructures, such as feeding hierarchies arising from gape-limited predation (Petchey et al., 2008; Woodward et al., 2010b). Recent explorations of so-called trivariate webs, in which 
feeding links are overlaid on mass-abundance plots, in marine (O'Gorman et al., 2010), freshwater (Jonsson et al., 2005; Layer et al., 2010; Woodward et al., 2012) and terrestrial (McLaughlin et al., 2010; Mulder et al., 2011) systems have revealed strong size structure. Typically, energy flows from many abundant, small resources to fewer, rarer and larger consumer species, with many webs containing one or a few apex predators but orders of magnitude more than basal species. These properties play an important stabilising role in the face of species loss and other perturbations (McLaughlin et al., 2010; O'Gorman et al., 2010), and could be especially important in fragmented habitats (Ledger et al., 2012; Woodward et al., 2012), where dispersal ability is also linked to body size. While seemingly ubiquitous in food webs, these patterns have yet to be described for mutualistic or host-parasitoid networks. More recently, body size, abundance, biomass and link data have been used to assess a range of substructural properties in aquatic food webs (Cohen et al., 2009), including tritrophic interactions (i.e. the smallest modular substructure beyond species pairs) and other recurring motifs (Woodward et al., 2012). Given that network substructure is likely to be related to both body size and spatiotemporal context, future work needs to focus on the potential impact of habitat fragmentation on the robustness of the underlying structural mechanisms in food webs and mutualistic networks, although species traits (e.g. abundance) other than size might be more important in the latter (but see Stang et al., 2006, 2009).

\subsubsection{Species abundance as a driver of ecological network structure}

Studies of ecological networks mostly focus on interactions among species (e.g. network references in Bascompte et al., 2003; Olesen et al., 2007). Individuals are the entities that are actually interacting, however, and as such their encounter rates, sensitive to habitat fragmentation, drive network structure (e.g. Petchey et al., 2010; Vázquez et al., 2009). For instance, flower abundance can account for much of the variation in linkage level of plants in pollination networks (Stang et al., 2006; but see Olesen et al., 2008). The importance of abundance for the functional roles of species in antagonistic networks is well known, but remains largely unexplored in mutualistic networks. Often a few common species engage in many interactions, and most rare species engage in few interactions (e.g. Memmott, 1999). This skewed structure affects several network metrics including nestedness, connectance and asymmetry (e.g. Blüthgen et al., 2008), although sampling artefacts need to be ruled out (Fischer and 
Lindenmayer, 2002; Lewinsohn et al., 2006; Vázquez, 2005; Vázquez et al., 2007; Woodward et al., 2010b). The effects of spatiotemporal changes in abundances on network structure remain relatively underexplored, but they are potentially key issues in the context of habitat fragmentation.

\subsubsection{Functional groups in ecological networks}

Species within functional groups (Hobbs et al., 1995; Körner, 1993) may be redundant, which is critical to network persistence under species extinction scenarios (Kaiser-Bunbury et al., 2010; Memmott et al., 2004) and other perturbations (Aizen et al., 2008; Kaiser-Bunbury et al., 2011; Tylianakis et al., 2007). The species traits that determine functional groups in ecological networks can differ within and between types of networks. In pollination networks, functional diversity defined by morphological traits might be vital for the persistence of diverse plant communities (Fægri and van der Pijl, 1979; Fontaine et al., 2006) and can constrain interaction patterns (Stang et al., 2006). In addition, functional groups can also be defined by behavioural traits (e.g. generalist vs. specialist), lifespan and temporal activity (e.g. seasonality of occurrence), phylogeny (similar roles of closely related species) and place of origin (e.g. native vs. exotic), which can influence pollination rates and species interactions (Fishbein and Venable, 1996; Kandori, 2002; Raine and Chittka, 2005), or whole pollination networks (Lopezaraiza-Mikel et al., 2007). Still remarkably little is known about how relative abundance affects within-functional group competition for the same resources at the network level.

\subsection{Spatial networks}

The analysis of multispecies ecological networks in a spatially explicit setting is still in its infancy (Dale and Fortin, 2010; Kissling et al., 2012a), although other types of networks have been investigated in spatial and landscape ecology (Dale and Fortin, 2010). Here, nodes are considered as locations (such as lakes or habitat fragments) and links define the connections among them (Dale and Fortin, 2010). The nodes (e.g. habitat fragments) have spatial coordinates and additional attributes related to size, shape, habitat quality and so on. The links among them can be defined by their distance or weight (e.g. measures of similarity in species composition among locations). Links are usually bidirectional (i.e. symmetric), but they can also be unidirectional, for instance, when the connection between lakes is represented by water flow. Spatial networks can thus form a conceptual basis for adding functional interrelations to habitat 
connectedness and physical structure to ecosystems (Dale and Fortin, 2010; Urban et al., 2009).

In a habitat fragmentation framework, spatial networks can quantify the effects of losing nodes or links, for example, by mimicking the loss of habitat patches or dispersal corridors for a single species within a meta-population (Urban and Keitt, 2001; Urban et al., 2009). More complex measures of species-specific landscape features, such as least-cost paths that describe the movement of a species through a heterogeneous matrix habitat, can also be integrated (Fall et al., 2007). The analysis of spatial networks in a static landscape (Urban and Keitt, 2001) can be extended to capture dynamic landscape processes that influence the persistence of patchy populations (Fortuna et al., 2006). Island biogeography perspectives (MacArthur and Wilson, 1967) can also be applied where separate fragments are seen as ecological islands embedded in a matrix of varying hostility.

Fragments can be connected via species that are present in both, creating a bimodal (rather than a one-mode) network of fragments and species. Roles can then be assigned to species and fragments according to their topological role and position in the network (Carstensen and Olesen, 2009; Guimerà and Amaral, 2005). Carstensen et al. (2012) used such an approach on a large scale and identified island roles and modules on the basis of shared avifaunas (i.e. biogeographic regions) and island characteristics.

\subsection{Combining spatial and ecological networks}

Regardless of whether it is possible to estimate landscape connectivity for all interacting species or for only a few key species, an integrative approach between spatial and ecological networks is needed to evaluate population persistence in fragmented landscapes (Gonzalez et al., 2011). This depends not only on the amount of habitat and its distribution in the landscape, but also on the position of each species within the ecological network (Solé and Montoya, 2006). For instance, top predators are particularly vulnerable to extinction in fragmented landscapes (Holyoak, 2000). Both spatial and ecological networks have similar concepts and are analysed with similar tools (Gonzalez et al., 2011), and integrating these into a single framework offers a promising way to advance the field (Dale and Fortin, 2010; Fortuna and Bascompte, 2008; Gonzalez et al., 2011; Olesen et al., 2010b).

Following Dale and Fortin (2010), a 'graph of graphs' can represent ecological network properties (e.g. nestedness of a plant-animal network) as nodes of a spatial network. In this way, one possibility is to view each local 
population as a node in a network with two kinds of links: (i) dispersal of individuals between fragments (local populations) and (ii) interactions between individuals of different species (e.g. pollination). The first kind of link provides an evaluation of landscape connectivity or habitat availability (Pascual-Hortal and Saura, 2006) for each species and the second kind gives the role each species plays in the ecological network of species interactions, such as its degree, centrality or contribution to nestedness. In this way, a value of habitat availability at the landscape scale may be assigned to each species plus a measure of its role in the ecological network(s), information that can be combined to evaluate its persistence probability. Moreover, different spatial configurations of habitats in the landscape and different arrangements of ecological networks can be modelled to estimate the impacts of fragmentation on persistence probabilities.

Recent theoretical studies illustrate the potential of unexpected consequences of the interplay between spatial and ecological networks by exploring three-species food chains. As a simple example, we may consider a tritrophic chain (Hastings and Powell, 1991) where a top predator Z feeds on an intermediate predator $\mathrm{Y}$ and on a prey $\mathrm{X}$, whereas $\mathrm{Y}$ feeds only on $\mathrm{X}$, with interactions ordered by body size $(Z>Y>X)$. The local extinction of $\mathrm{Y}$ in small patches jeopardises the survival of the large predator $\mathrm{Z}$ and may lead to a overpopulation of $X$. Examples of outbreaks in spatially distributed populations have indeed been described theoretically (Araújo and de Aguiar, 2007; Maionchi et al., 2006), showing that probable reduction in abundance of intermediate species may have important indirect ramifications for other species via their interactions in the ecological network. Recent experimental work shows that although intermediate species may be lost, it is often the larger species at the terminus of tritrophic chains that are especially prone to local extinctions due to habitat fragmentation, leading a reduction in the trophic level of the web as a whole (Woodward et al., 2012).

Theoretical studies further indicate that dynamical instabilities caused by large dispersal abilities of predators, relative to their prey, in spatial networks create abundance heterogeneities among otherwise equivalent fragments (Mimura and Murray, 1978; Nakao and Mikhailov, 2010; Rietkerk et al., 2004). These so-called Turing patterns (Murray, 1993; Rietkerk et al., 2004; Turing, 1952) represent the combined effect of species dispersal, interactions and spatial configuration. They may also have indirect consequences on other species by altering the composition of potential prey, predators, competitors and mutualistic partners in ecological networks among fragments. Such explorations of the interplay 
between spatial and ecological networks highlight the need to focus on understanding how fragmentation affects population dynamics within multispecies systems.

\section{HABITAT FRAGMENTATION}

\subsection{General introduction}

Habitat fragmentation is often defined as a process during which a large expanse of habitat is transformed into a number of patches of a smaller total area, isolated from each other by a matrix of habitats unlike the original (Wilcove et al., 1986). It increases discontinuity in the spatial patterning of resource availability, affecting the conditions for species occupancy, and ultimately individual fitness. Fragmentation can arise via both natural and anthropogenic processes in terrestrial and aquatic systems (Figs. 1 and 3). In the latter, fragmentation affects freshwaters (e.g. rivers and lakes) as well as marine systems (e.g. oceans, coral reefs, seagrass meadows, kelp forests, salt marshes and sea ice) (Box 1). In terrestrial systems, habitat fragmentation can be induced by many drivers, including lava flows and the conversion of forest to farmland (either grasslands or arable fields). Our focus is primarily on anthropogenic fragmentation of pristine habitats, which is occurring at an accelerating rate on a global scale. An illustrative example of the effect of habitat fragmentation in the Atlantic Rainforest of Brazil is provided in Box 2.

The effects of fragmentation on biodiversity depend on specific species traits and characteristics of the fragments and the surrounding matrix (Ewers and Didham, 2006; Fahrig, 2003; Henle et al., 2004). At least four effects form the basis of most quantitative measures of habitat fragmentation (Fahrig, 2003): (a) reduction in habitat amount, (b) increase in the number of fragments, (c) decrease in fragment size and (d) increase in fragment isolation. While habitat loss per se will reduce population sizes and, ultimately, the loss of species and their links (Bierregaard et al., 1992; Fahrig, 2003; Franklin and Forman, 1987; Saunders et al., 1991), fragmentation includes a much wider array of patterns and processes and far more complex consequences for biodiversity. We will review the importance of fragment characteristics (size and isolation, including connectivity and corridors), habitat edges (including edge permeability and geometry) and matrix quality, before discussing spatial and temporal turnover and the importance of scale. 


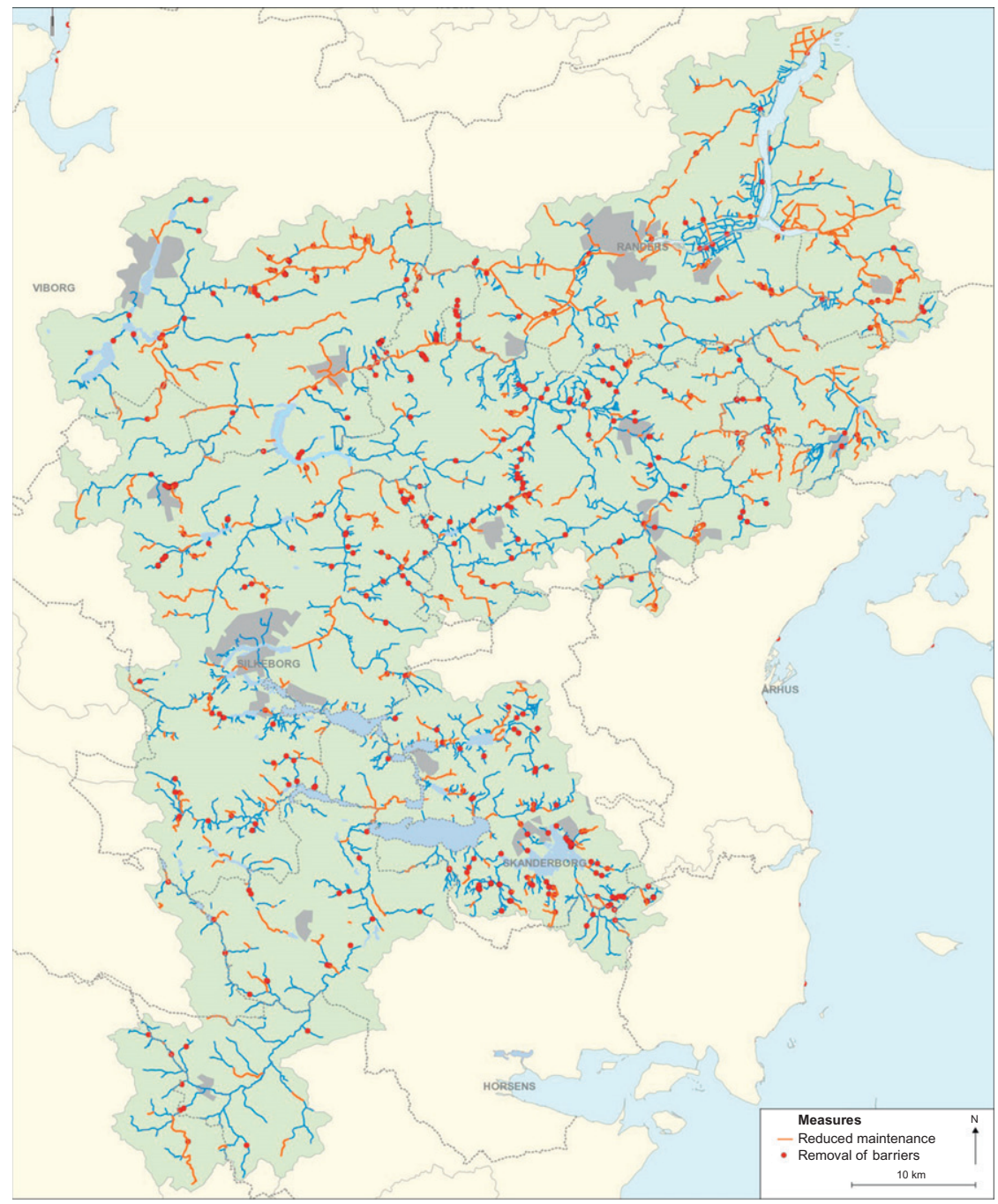

Figure 3 Anthropogenic fragmentation of a European river network (Denmark, E. Jutland, the city of Aarhus at the bay-center-right of map; map size: E-w 80km). Dots indicate physical barriers (weirs, dams, impoundments) to fish migration, a major source of human-mediated impacts (Feld et al., 2011). The map of the Gudenå catchment, is derived from the River Basin Management Plan, reproduced courtesy of The Danish Ministry of Environment. 


\section{BOX 1 Habitat Fragmentation in Aquatic Ecosystems}

Fragmentation plays a key role in both terrestrial and aquatic ecosystems, including freshwater, estuarine and marine systems (e.g. oceans, coral reefs and seagrass meadows).

Freshwaters are commonly viewed as being bounded by hard edges as they are 'fragmented islands in a terrestrial sea' (Woodward and Hildrew, 2002a), but they also have soft boundaries within their borders (Figs. 5 and 16) imposed by chemical gradients such as $\mathrm{pH}$ or salinity, especially where they mix with coastal waters in estuaries. Human activity has accelerated the rate and extent of fragmentation in freshwaters, particularly by overabstraction of water by growing populations (Vörösmarty et al., 2010). Climate change is also to exacerbate hydrological droughts (Poff and Zimmerman, 2010) via reduced rainfall in many areas (Kundzewicz et al., 2008), potentially causing widespread habitat loss and fragmentation (Boulton, 2003; Lake, 2003; Ledger et al., 2011). During droughts, river flows decline, reducing the volume of wetted habitat (water width and depth) and altering habitat structure, increasing water temperature, reducing dissolved oxygen (Everard, 1996) and altering nutrient supply (Dahm et al., 2003). In some regions, droughts occur predictably as part of the natural hydrologic cycle and species are able to tolerate such conditions (Bonada et al., 2007), but elsewhere unpredictable drought fragmentation can have devastating effects on aquatic food webs (Ledger et al., 2011).

Marine systems such as oceans, coral reefs and seagrass meadows are also exposed to fragmentation. For instance, the open ocean might appear to be relatively homogenous, but there are distinct vertical and horizontal regions separated by physicochemical barriers, such as pycnoclines and frontal systems, which are more permeable to larger organisms (e.g. anadromous and catadromous fishes) than to the smaller organisms. Coral reefs experience increased rates of habitat loss and fragmentation due to dynamite fishing (Fox, 2004; Raymundo et al., 2007; Riegl and Luke, 1998; Wells, 2009), and coral bleaching is occurring with increasing frequency due to rising sea temperature (Oliver and Palumbi, 2009). The loss of structural complexity in these fragmented coral landscapes results in declining abundances and diversities of reef fish and mobile invertebrates (Bonin et al., 2011; Coker et al., 2009; Graham et al., 2007; Pratchett et al., 2008; Syms and Jones, 2000). Local extinctions are proportionally greater for resource specialists than generalists (Munday, 2004). Other marine systems include seagrass meadows, which form unique, productive and diverse ecosystems (Bostrom et al., 2006; Duarte and Chiscano, 1999). They are affected by fragmentation through dredging and boating effects, eutrophication, extreme weather events, urchin grazing and wasting disease (Bostrom et al., 2006; Orth et al., 2006; Rasmussen, 1977; Walker and 


\section{BOX 1 Habitat Fragmentation in Aquatic Ecosystems-cont'd} McComb, 1992; Walker et al., 2006). While many studies suggest that fragmentation of seagrass meadows has limited (Frost et al., 1999; Hirst and Attrill, 2008; MacReadie et al., 2009), inconsistent (Bell et al., 2001) or even positive (Eggleston et al., 1998; Hovel and Lipcius, 2001) impacts on epifaunal diversity and abundance, fragmentation beyond a threshold level can lead to rapid declines in species diversity and abundance (Reed and Hovel, 2006).

Other major marine habitats influenced by fragmentation include kelp forests, salt marshes and sea ice. Habitat loss in kelp forests reduces biomass and abundance of fish (Deza and Anderson, 2010). The die-off of salt marshes results in changes in the behaviour of key grazers (snails) as they seek shelter from predation by blue crabs (Griffin et al., 2011; Silliman et al., 2005). Finally, increased fragmentation of sea ice habitats results in declines in mating success and searching efficiency of top predators such as polar bears (Molnár et al., 2011) and in changes in phototrophic community structure and relative abundance of dominant marine taxa (Mueller et al., 2006).

\section{BOX 2 Habitat Fragmentation and its Effect on Brazilian Atlantic Rainforest Trees}

A good example of a biodiversity hotspot affected by fragmentation is the Brazilian Atlantic rainforest landscape, which is dominated by a mosaic of small forest fragments usually embedded in a heterogeneous matrix of urban and agricultural land (Ribeiro et al., 2009). The abundance and diversity of many taxa (including frogs, lizards, small mammals and birds) are generally positively affected by the surrounding matrix (Pardini et al., 2009, and see also Faria et al., 2006, 2007), whereas the richness and abundance of shade-tolerant trees are negatively affected and decline from large to small fragments (Pardini et al., 2009). This indicates that increasing landscape heterogeneity might allow the maintenance of higher diversity of animals, but that specialist tree species depend on the maintenance of native forest patches (Pardini et al., 2009; Ribeiro et al., 2009). In the more extreme scenario of a hyper-fragmented Northeast Brazilian Atlantic forest (i.e. a landscape composed of pastures, monoculture plantations and a few small native forest fragments), tree species and reproductive trait diversity are lost (Lopes et al., 2009; Oliveira et al., 2008), whereas early successional trees can proliferate in small forest remnants (Tabarelli et al., 2008). An expansion of pioneer species in the edge dominated habitats can be associated with changes in functional reproductive traits, diurnal pollination systems, and loss of long-distance flying pollinators, self- 


\section{BOX 2 Habitat Fragmentation and its Effect on Brazilian Atlantic Rainforest Trees-cont'd}

incompatible breeding systems and large-seeded plant species. Furthermore, phenological trait mismatches can occur, due to shifts in the proportions annual versus supra-annual flowering (Lopes et al., 2009; Santos et al., 2008; Tabarelli et al., 2010). Modelling efforts predict a pervasive long-term trend towards vegetation dominated by early successional trees and impoverished tree species composition (Pütz et al., 2011), with important implications for plant-animal mutualistic networks. Specialised and long-distance moving connector species in mutualistic networks such as large pollinators (bees or hummingbirds) and seed dispersers (large birds) are likely to be particularly vulnerable due to reduced floral diversity and quality arising from the dominance of generalist pollination systems, and the large proportion of species that are wind dispersed or which have small fleshy fruits (e.g. Lopes et al., 2009; P. Morellato, unpublished data).

\subsection{Fragment characteristics}

Fragment characteristics are important for understanding fragmentation effects on biodiversity (Table 1). Apart from original habitat loss per se (Tilman et al., 1994), size (i.e. area) and degree of isolation of fragments are important properties (Fahrig, 2003). For some taxa such as butterflies, habitat heterogeneity seems to be a more important determinant of diversity than fragment size and isolation (Kivinen et al., 2006; Rundlöf and Smith, 2006; Weibull et al., 2000), and this may be true for other herbivorous insects as well.

The area needed to maintain populations is determined by fragment size, with smaller patches generally containing fewer individuals and species than larger patches (Debinski and Holt, 2000). The area effect on biodiversity can be predicted from species-area curves (Sabatino et al., 2010), and the set of species in smaller patches is often a fairly predictable subset of those in larger patches (nested structure; e.g. Ganzhorn and Eisenbeiss, 2001; Hill et al., 2011). Species richness in forest fragments in relation to fragment area (Brooks et al., 1997; Ewers and Didham, 2006) can mirror the classic species-area relationships known from island biogeography (MacArthur and Wilson, 1967). To some extent, temporal effects are also dependent on fragment size because what happens quickly in small fragments happens slowly in larger fragments (Terborgh et al., 1997). 
Table 1 Fragment characteristics and animal and plant traits, which are relevant for assessing fragmentation effects on biodiversity Trait Importance of trait in relation to fragmentation

References

Trait at fragment level

Size (area) The size of fragments determines the area available for population and species persistence and influences extinction and immigration rates

Bender et al. (1998), Fahrig (2003),

MacArthur and Wilson (1967)

\begin{tabular}{lll}
\hline Isolation & $\begin{array}{l}\text { The degree of isolation of fragments represents the lack of habitat in } \\
\text { the surrounding landscape and has an influence on the movement and } \\
\text { dispersal of species among fragments }\end{array}$ & $\begin{array}{l}\text { Ewers and Didham } \\
(2006), \text { Fahrig (2003) }\end{array}$ \\
\hline Shape & $\begin{array}{l}\text { Convoluted fragment shapes can lead to increased turnover and variability in } \\
\text { population size when compared to fragments that are compact in shape }\end{array}$ & $\begin{array}{l}\text { Ewers and Didham } \\
(2006)\end{array}$ \\
\hline Edge effects & Edges of fragments affect microclimate and animal abundances & Laurance et al. (2011) \\
\hline Matrix effects & $\begin{array}{l}\text { The surrounding matrix mediates edge effects and influences animal (e.g. } \\
\text { pollinator and seed disperser) movements }\end{array}$ & Laurance et al. (2011) \\
\hline Animals & & $\begin{array}{l}\text { Ewers and Didham } \\
\text { Species with high mobility are more likely to survive in fragmented } \\
\text { landscapes than species with low mobility. Low mobility or poor dispersal } \\
\text { ability of species is thus expected to increase species-level fragmentation } \\
\text { effects. For some butterflies, it has been shown that species with } \\
\text { intermediate mobility are more likely to decline in abundance following } \\
\text { habitat fragmentation than species with either high or low mobility }\end{array}$ \\
\hline
\end{tabular}


Table 1 Fragment characteristics and animal and plant traits, which are relevant for assessing fragmentation effects on biodiversity-cont'd Trait Importance of trait in relation to fragmentation

\section{References}

Habitat

specialisation

Habitat specialists are expected to be more affected by fragmentation than role here

(e.g. forest generalist vs. habitat generalist)

Trophic level Higher trophic levels are predicted to be more strongly affected by habitat fragmentation than lower trophic levels habitat generalists. The matrix tolerance of a species might play an important

Ewers and Didham

(2006)

Ewers and Didham (2006), Milton and May (1976)

\begin{tabular}{lll}
\hline $\begin{array}{l}\text { Dietary } \\
\text { specialisation }\end{array}$ & $\begin{array}{l}\text { Species with broad dietary niches might be less impacted by fragmentation } \\
\text { than dietary specialists }\end{array}$ & Bommarco et al. (2010) \\
\hline $\begin{array}{l}\text { Gap-crossing } \\
\text { ability }\end{array}$ & $\begin{array}{l}\text { Species persistence in isolated fragments is strongly linked to gap-crossing } \\
\text { ability }\end{array}$ & Lees and Peres (2009) \\
\hline Body size & $\begin{array}{l}\text { Body size constrains animal space use and home range size. Home range size } \\
\text { is expected to increase with habitat fragmentation, and home ranges of larger }\end{array}$ & $\begin{array}{l}\text { Greenleaf et al. (2007), } \\
\text { species are more sensitive to habitat fragmentation than those of smaller } \\
\text { et al. (2004), Laurance } \\
\text { et al. (2011) }\end{array}$ \\
\hline species & $\begin{array}{l}\text { Sociality can buffer against negative effects of fragmentation } \\
\text { (e.g. social bees vs. solitary bees) or increase susceptibility to fragmentation }\end{array}$ & $\begin{array}{l}\text { Aizen and Feinsinger } \\
(1994 a, b), \text { Bommarco } \\
\text { et al. (2010), Laurance } \\
\text { et al. (2011) }\end{array}$ \\
\hline
\end{tabular}


Table 1 Fragment characteristics and animal and plant traits, which are relevant for assessing fragmentation effects on biodiversity-cont'd Trait Importance of trait in relation to fragmentation

References

Plants

Dispersal mode Dispersal mode (e.g. abiotic vs. biotic) can be a key factor influencing species responses to habitat fragmentation

Montoya et al. (2008) Tabarelli et al. (1999), Tabarelli and Peres (2002)

\begin{tabular}{ll} 
Fruit/seed size & $\begin{array}{l}\text { Large big-seeded fleshy fruits tend to have few dispersal agents and are likely to } \\
\text { be more strongly affected by fragmentation than plant species with small fleshy } \\
\text { fruits }\end{array}$ \\
\hline $\begin{array}{l}\text { Pollination } \\
\text { mode }\end{array}$ & $\begin{array}{l}\text { Plants depending on animals for pollination are probably negatively affected } \\
\text { by habitat fragmentation (specifically isolation) than wind-pollinated species }\end{array}$
\end{tabular}

Corlett (1998) by habitat fragmentation (specifically isolation) than wind-pollinated species

Aizen and Feinsinger (1994a,b), Fægri and van der Pijl (1979), Kolb and Diekmann (2005)

\begin{tabular}{lll}
\hline $\begin{array}{l}\text { Breeding } \\
\text { system }\end{array}$ & $\begin{array}{l}\text { Characteristics of breeding systems, for example, the degree of protandry, } \\
\text { self-incompatibility or sex ratios, might be affected by fragmentation }\end{array}$ & $\begin{array}{l}\text { Jennersten (1988), Yu } \\
\text { and Lu (2011) }\end{array}$ \\
\hline Growth form & $\begin{array}{l}\text { Specific growth forms (e.g. clonal plants) might be more strongly affected than } \\
\text { others (e.g. annuals) }\end{array}$ & $\begin{array}{l}\text { Dupré and Ehrlén } \\
(2002), \text { Kolb and } \\
\text { Diekmann (2005) }\end{array}$ \\
\hline Seed bank & $\begin{array}{l}\text { Long-lived seed banks may prevent species from going extinct in small } \\
\text { habitat fragments }\end{array}$ & $\begin{array}{l}\text { Dupré and Ehrlén } \\
(2002)\end{array}$
\end{tabular}

The list highlights some key traits but is not intended to be exhaustive. 
Isolation restricts the movement and dispersal of species among fragments and depends on physical distance and matrix quality (Bender et al., 2003). Two aspects of fragment isolation are particularly important: connectivity and the availability of corridors. Connectivity is the degree to which the landscape permits or impedes movement among fragments (Taylor et al., 1993) and is a species and system-specific parameter (Taylor et al., 2006; Tischendorf and Fahrig, 2000). Its effect on biotic interactions (e.g. pollination services) is therefore a complex function of the individual responses of the different interacting species (for pollinators see e.g. Fenster and Dudash, 2001; Herrera, 1988; Horvitz and Schemske, 1990; Moeller, 2005; Ricketts et al., 2006). Some species may primarily be influenced by the distance to a fragment of a particular habitat, while others may be more influenced by the quality or availability of the resource (e.g. nesting sites) in adjacent habitats. One structural landscape characteristic of high importance for connectivity is the presence of corridors, which can be either natural or man-made. They are landscape elements that facilitate the movement of organisms among fragments, promoting biotic connectivity and synchrony (Hilty et al., 2006). Recent experiments have demonstrated that corridors play a key role in maintaining plant and animal populations and their interactions in fragmented landscapes, and that connected fragments retain more species from native biota than isolated ones (Damschem et al., 2006; Tewksbury et al., 2002). Their importance for biodiversity conservation is still a moot point (Gilbert-Norton et al., 2010; Noss, 1987; Simberloff and Cox, 1987; Simberloff et al., 1992), as in some systems (e.g. tropical rainforests) corridors and fragments dominated by secondary vegetation may be of limited value (Oliveira et al., 2008).

\subsection{Habitat edges}

Increased edge habitats, which may be natural (e.g. light gaps, rivers and landslides in natural forests) or anthropogenic, are prominent features of a fragmented landscape. Habitat edge and fragment shape are important determinants of biodiversity (Ewers and Didham, 2006; Laurance et al., 2011; Murcia, 1995), and strong effects on a variety of plant and animal species are well documented (e.g. Bach and Kelly, 2004; Davies et al., 2000; Gehlhausen et al., 2000; Laurance et al., 1998).

Three main physical and biological effects of edges are important in fragmented habitats (Murcia, 1995): (i) abiotic environmental changes across edges; (ii) biological effects related to changes in species in the edge and 
across the edge as a result of (i), and (iii) indirect biological effects, which relate to how changes in (ii) cascade up and affect species via their antagonistic and mutualistic interactions.

Changes in abundance across a habitat edge depend on the taxonomical/ functional groups involved. Generalist species are often favoured in habitat edges, because they offer access to new habitats and resources (e.g. pollinators: Burgess et al., 2006, herbivores: Wirth et al., 2008, predators and nest predation: Chalfoun et al., 2002; Lidicker, 1999), whereas specialists typically decline (plants: Laurance et al., 1997, 2006a; Tabarelli et al., 2008, insectivorous birds: Restrepo and Gómez, 1998, vertebrates: Hansson, 1994, but see Pardini et al., 2009 for a multi-taxa approach).

Species that require different habitat types for different resources or life history stages (e.g. nesting, feeding and foraging) are expected to benefit from a structurally diverse habitat mosaic (including edges). For example, solitary bees that nest above-ground forage in agricultural landscapes, but nest in neighbouring natural habitats (Gathmann and Tscharntke, 2002). Aquatic insects often rely on trees as 'swarm-markers' for breeding once they have emerged from the water and crossed the aquatic-terrestrial boundary. Similarly, riparian vegetation provides the main source of energy to many stream food webs in the form of terrestrial leaf-litter, so the proximity to this edge can determine the trophic basis for production for the entire system (Hladyz et al., 2011b). Even predators can benefit from inputs from terrestrial edges, with such subsidies supporting some stream fishes at densities far beyond what in-stream production alone can support (Allen, 1951). Edges also influence seed banks and the quality, abundance and diversity of seed rain (Devlaeminck et al., 2005, Melo et al., 2006).

In forests, especially tropical ones, the increasing air temperature, light incidence and decreasing relative humidity towards the edge (Didham and Lawton, 1999; Kapos et al., 1997; Murcia, 1995) can affect plant reproduction by shifting phenology and boosting flower and fruit production (Burgess et al., 2006; Camargo et al., 2011; D’Eça Neves and Morellato, in press; Kato and Hiura, 1999; Murcia, 1995) (Fig. 4). In turn, important animal-plant interactions can be affected (Aizen and Feinsinger, 1994a,b; Cunningham, 2000; Fleury and Galetti, 2006; Galetti et al., 2006; Jordano and Schupp, 2000; Wright and Duber, 2001). Pollination rates at edges may decrease (Aizen and Feinsinger, 1994a,b; Burgess et al., 2006; Harris and Johnson, 2004; Hobbs and Yates, 2003), increase (Burgess et al., 2006), or may not change at all (Burgess et al., 2006), with implications for plant reproductive success 
A

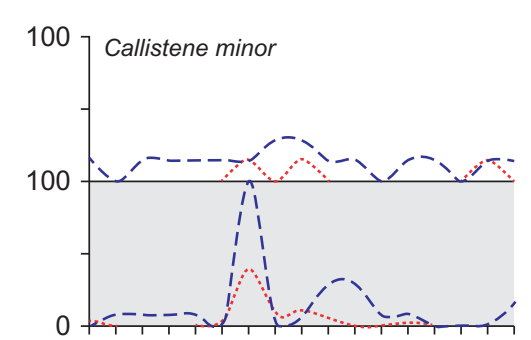

C

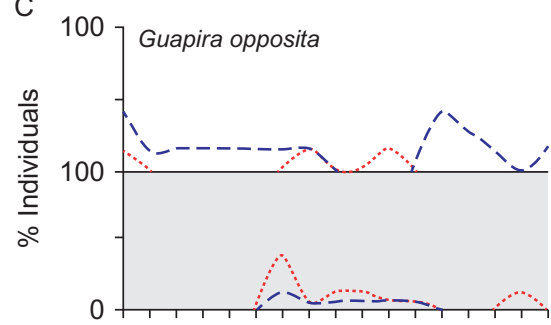

E

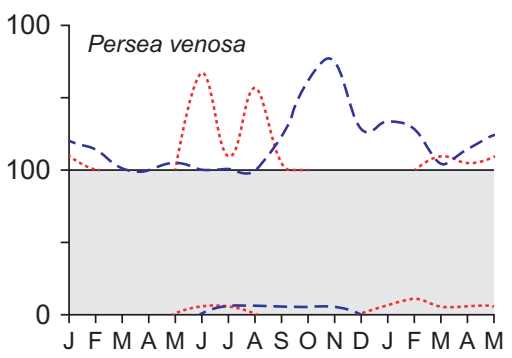

B

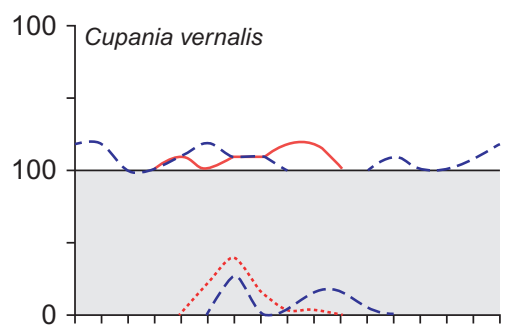

D

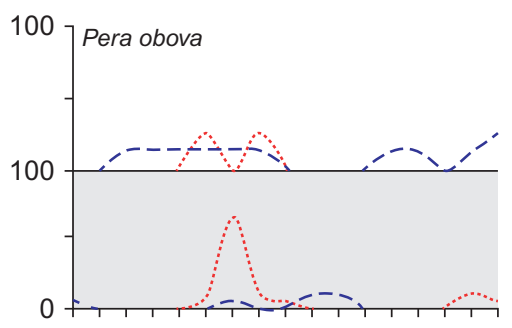

$\mathrm{F}$

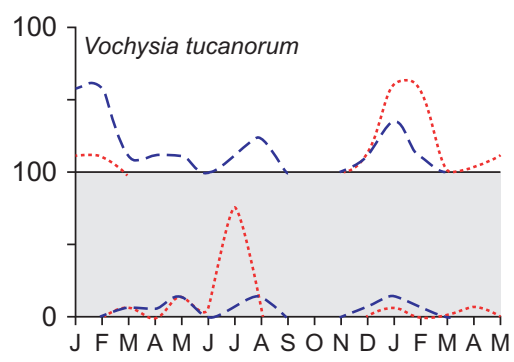

Flower ---- Fruit

Figure 4 Phenological response of trees occurring in the edge (shaded graphics) and interior of a seasonal forest at Serra do Japi, Southeastern Brazil (after D'Eça Neves and Morellato, in press). Positive responses (higher proportion of trees) for flowering were detected in four of six species (Figure edge $A$ to $D$ ). On the other hand, negative edge effects on fruiting were detected for four species (Figure edge $C$ to F). Although the fruit production of the woody Cupania vernalis (Sapindaceae) was positively affected by fragment edge (Figure edge B), Guimarães and Cogni (2002) observed a higher seed predation of $C$. vernalis in the edges at the same study site. Therefore, differential phenological responses at the edges may change the visitation rates of pollinators, dispersers and seed consumers, making it hard to predict the reproductive outcome to the plant.

(Burgess et al., 2006; Cunningham, 2000) and seed dispersal. The influence on the latter may be either positive due to differences in animal densities, foraging patterns, fruit display, plant size and vigour (Jordano and Schupp, 2000), or negative via limited animal movement at edges 
(Restrepo et al., 1999). Furthermore, recruitment and predation of seeds in the forest interior might decrease relative to edges (Baldissera and Ganade, 2005; Fleury and Galetti, 2006; Jules and Rathcke, 1999; Restrepo and Vargas, 1999, but see Cunningham, 2000; Guimarães and Cogni, 2002).

Besides the capability of a species to perceive suitable habitat fragments and the connectivity of the landscape, its persistence in a fragmented landscape depends on its ability to cross the edge between fragment and matrix (Morris, 1997; Stamps et al., 1987a; Stevens et al., 2006). Habitat edges can be characterised as 'hard' or 'soft' according to their permeability. Hard edges are boundaries which dispersing individuals rarely (if ever) cross, although their permeability can vary with life history, for example, adults or juveniles (Fig. 5). Soft edges are more permeable: for example, bumblebees (Bombus hortorum) cross several habitat edges between meadows, fields and gardens and move widely within a mosaic landscape (Hagen et al., 2011). Changes in edge permeability (e.g. due to degradation of the landscape matrix around a fragment) can alter migration rates, as well as several other ecological and demographic processes. For instance, population densities within the fragment may be elevated, maturity delayed, and reproductive and growth rates reduced (Abramsky and Tracy, 1979, 1980; Gliwicz, 1980; Lidicker, 1985; Myers and Krebs, 1971; Stamps et al., 1987b).

Emigration rates (i.e. the proportion of dispersing individuals that leave the fragment) from habitat fragments are also determined by the edge-to-size ratio and the shape of the habitat edge (Nams, 2011). For instance, Hardt and Forman (1989) found forest herbivores to concentrate in the grassy areas where the edge intrudes into the forest. Some pollinating bee species (e.g. Bombus lapidarius; Rasmussen and Brødsgaard, 1992) avoid edges while foraging for pollen within fragments, while responses of birds to edges vary markedly among species and edge types (Sisk and Battin, 2002).

\subsection{Matrix}

The matrix surrounding fragments also influences their structure and dynamics (Brotons et al., 2003; Cook et al., 2002; Prevedello and Vieira, 2011; Prugh et al., 2008). Among forest fragments, matrix quality can range from a completely deforested agricultural landscape to mature secondary growth, varying immensely in hostility and permeability to each species. Matrix quality thus determines connectivity, dispersal and associated mortality rates, and its influence may even override those of 


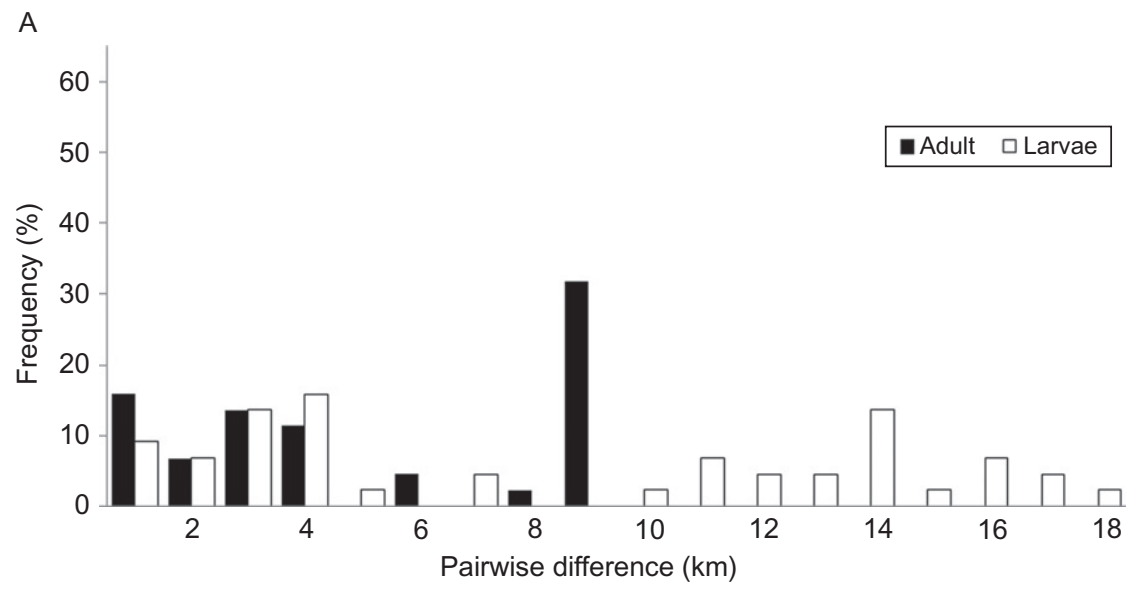

B

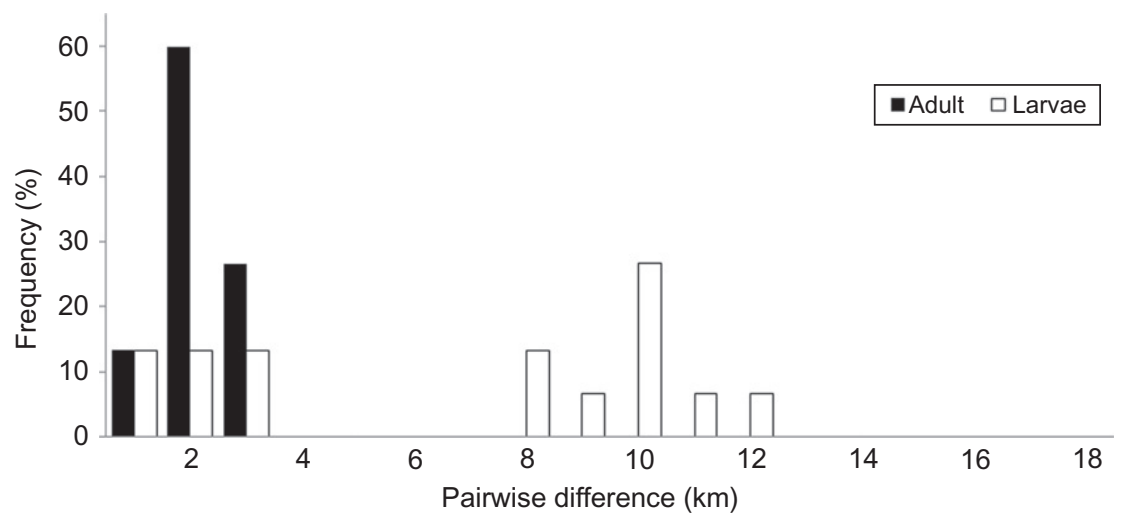

Figure 5 Frequency distribution of pairwise distances for all individual streams in the Ashdown Forest network, Sussex, UK (Fig. 16), for both adult winged insects (solid black bars) and immature aquatic insects and other solely aquatic organisms, including fishes, molluscs, Crustacea and other groups (white bars). (A) The River Medway network and (B) the River Ouse network. Note: inter-catchment exchange is not included here, since although it is feasible in the aerial phase, none of the solely aquatic taxa in these webs are able to cross the marine-freshwater boundary, which acts as a 'hard' boundary for all the insect species that dominate these food webs. Aquatic invertebrates are incapable of crossing from one network to the other, due to the lack of suitable corridors. Fewer than $1 \%$ of all fish species can make the transition between fresh and salt water (brown trout and common eels are the only notable exceptions within the river networks shown here), so for many taxa these two catchments are in reality separated by 100 s of kilometres of an insurmountable physicochemical barrier even though the local webs may be just a few kilometres apart in the upper headwaters. There is also likely to be an evolutionary spatiotemporal component to fragmentation here, as these catchments have likely been flowing in different directions and hence effectively isolated for many taxa since the retreat of the ice sheets at the end of the last glaciation. 
fragment area and isolation (Cook et al., 2002; Ewers and Didham, 2006). A high-quality matrix (e.g. forest regrowth) can minimise edge effects by supporting a proportion of the communities in the fragments (Laube et al., 2008; Pardini et al., 2009 and references therein).

A diverse and structurally complex, anthropogenic matrix may even harbour a significant fraction of the original biota, potentially reducing biodiversity loss (Lindenmayer and Luck, 2005; Pardini et al., 2009). For instance, in Western Kenyan rainforest, some bird species (11\% out of 194 forestdependent species; Bennun and Njoroge, 1999) also used the heterogeneous farmland close to the forest as feeding habitat, gaining access to additional food resources outside their core habitat (Laube et al., 2008). Thus, agroecosystems with a diverse habitat structure can have at least some capacity to compensate for forest loss. Indeed, several frugivorous bird species use native and exotic fruiting trees in the farmland around the same forest, increasing seedling establishment (Berens et al., 2008; Eshiamwata et al., 2006), suggesting the matrix can aid fragment regeneration and restoration (Fisher et al., 2010). Further, bee diversity is higher than in the nearby forest, so the farmland may even act as a 'pollinator rescue', supporting pollination services inside the forest (Hagen and Kraemer, 2010). Other studies have reported positive influences of natural forest on pollination interactions in farmland (e.g. Florida, USA: Artz and Waddington, 2006; North Queensland, Australia: Blanche et al., 2006).

Matrix quality can also be important for food webs. A recent study has shown how the invasion of the terrestrial edge habitat can cause a collapse in food web structure and ecosystem processes of an adjacent stream, by altering the porosity of energy flux across the ecotone (Hladyz et al., 2011a). Here, the native terrestrial matrix through which the stream would normally flow is either in the form of the mixed deciduous woodland climax community, or rough pasture maintained by low intensity farmland. The invasive tree Rhododendron ponticum forms dense, dark monocultures that outcompete native riparian plant species and cast a deep shade over the stream food webs. Invasions can occur within either of these starting conditions, although they are accelerated by anthropogenic disturbance along the aquatic-terrestrial fragment-matrix edge. Because the tough, leathery leaves of the invader are also a poor-quality food source, being very high in $\mathrm{C}: \mathrm{N}$ and lignin content (Hladyz et al., 2009), they effectively shut down the detrital pathway at the base of the stream food web, which is normally fuelled by leaf-litter when the matrix is dominated by oak woodland. The invader 
also suppressed the alternative energy source supplied by algal-herbivore pathways that would otherwise dominate when the terrestrial matrix is rough pasture, by shading the stream channel (Hladyz et al., 2011a). Consequently, invasive species can harden the fragment-matrix boundary, by reducing the permeability of energy transfer. Additional recent evidence from a pan-European study suggests that riparian alterations tend to suppress animal-resource interactions at the base of stream food webs, increasing reliance on microbial-driven rather than invertebrate-driven processes (Hladyz et al., 2011b).

\subsection{Spatial and temporal turnover of species and individuals}

Spatial and temporal turnover in species composition among habitats in a fragmented landscape can be pronounced. For instance, Hagen and Kraemer (2010) found high turnover rates in bee species composition between open farmland, forest-farmland edge and forest interior: almost $50 \%$ of all bee species in this landscape mosaic occurred in all three habitat types, indicating a high edge permeability or a so-called soft edge.

In contrast, in a European meadow, pollinator species did not cross the edge into the adjacent forest, whereas herbivores and pathogens did (L. Kromann-Gallop, personal communication). Shifts in behaviour (e.g. flower visitation rates) may also occur among individuals of the same species of pollinator, leading to differences in fruit and seed set among habitats (Kaiser et al., 2008). Additionally, the roles of species in an ecological network (e.g. peripherals, connectors, module hubs and network hubs; Olesen et al., 2007) can change when crossing habitat borders (M. Hagen et al., unpublished data). Of 35 species ( 8 plant and 27 bee species) occurring in all three habitats in a forest-agriculture landscape, 23 (3 plants, 20 bees) had similar roles in all habitats, as did 11 ( 4 plants, 7 bees) species in two of the three habitats, and one plant had a different role in each habitat.

Due to physical changes at habitat edges, phenological shifts in interactions may arise, resulting in a complex interplay between spatial and temporal turnover. Edges and interiors may therefore differ in the timing of resource availability and network structure and dynamics. Unfortunately, detailed data remain scarce (Kato and Hiura, 1999; Ramos and Santos, 2005), but an increase in flower production at forest edges associated with high light incidence and temperatures have been reported for some species (Alberti and Morellato, 2010; Camargo et al., 2011; Fuchs et al., 2003; Kato and Hiura, 1999; Ramos and Santos, 2005). D’Eça Neves and 
Morellato (in press) compared the phenology of tree species between forest edge and interior in Southeastern Brazil and found a higher proportion of reproductive trees along the forest edge (59\% flowering and 73\% fruiting) than inside the forest (47\% flowering and 29\% fruiting), and flowering and fruiting were more seasonal in the latter. As individual tree species can respond differently to edge effects (Fig. 4), the synchrony and degree of overlap between the interaction partners in an ecological network may be affected by this aspect of habitat fragmentation (e.g. Hegland et al., 2009; Memmott et al., 2007).

The predominance of generalism and seemingly high plasticity of interactions in many ecological networks may reduce the effects of spatial and temporal mismatches. The available literature, albeit scarce, indicates that pollination networks are fairly robust against such mismatches (see Hegland et al., 2009) and the same may be true for food webs, which are typically even more generalised (Ings et al., 2009). Plants and pollinators exposed to similar environmental changes may react in synchrony, decreasing the occurrence of mismatches (Hegland et al., 2009). In pollination networks, high turnover in species composition and interactions over time are well documented (Alarcón et al., 2008; Dupont and Olesen, 2009; Olesen et al., 2008; Petanidou et al., 2008), but the consequences of adding the spatial component of a fragmented landscape to temporal mismatches are virtually unknown.

\subsection{Scales of habitat fragmentation}

Fragmentation operates over many spatial and temporal scales (Levin, 1992), from tiny water bodies within individual plants (Phytotelmata; Box 3) to successional processes across entire landscapes, for instance, as stream networks develop following glacier retreat (Brown and Milner, 2012; Jacobsen et al., 2012; Woodward and Hildrew, 2002a). Individual organisms perceive the world at different spatial and temporal scales and thus will respond to fragment characteristics, habitat edges and matrix permeability in different ways. Within food webs, consumer-resource perceptual disparities may be pronounced, closely coupled to the relationship between body size and environmental grain: for example, single-celled algae and small invertebrates at the base of aquatic food webs are many orders of magnitude smaller than the large vertebrates at the top (e.g. Cohen et al., 2009; Layer et al., 2010). The immediate environment within which a diatom spends its (short)life attached to a substrate particle on a streambed is thus shaped largely by small-scale 


\section{BOX 3 Phytotelmata-Small Aquatic Worlds in a Highly Fragmented Landscape}

Phytotelmata (from Ancient Greek, meaning 'plant ponds') are small water bodies within plants that exist as aquatic refugia within a much larger terrestrial ecosystem. Examples include tree holes, bamboo internodes, pitcher plants, tank bromeliads and water-retaining plant axils (Kitching, 2001). Phytotelmata have been intensively studied as they represent naturally replicated systems containing discrete communities and food webs within individual plants (Reuman et al., 2009). The macrofaunal assemblages they contain can range from 2 to 20 species (Kitching, 2001) and are often dominated by arthropods, although annelids, frog tadpoles and molluscs have also been recorded (Kitching, 2000). In addition, they contain a diverse range of microscopic life, including rotifers, protozoa and bacteria (Buckley et al., 2010; Kneitel and Miller, 2002).

Phytotelmata can be regarded as insular systems (Kitching, 2001), and they have been useful models for testing island biogeography theory (MacArthur and Wilson, 1967). An investigation of the macrofaunal diversity in epiphytic bromeliads shows that species richness increases with phytotelma size and physical habitat complexity (Armbruster et al., 2002; Buckley et al., 2010; Srivastava, 2006). Phytotelmata are extremely isolated as the surrounding matrix (e.g. terrestrial forest) is hostile. There is no connectance between phytotelmata via corridors, so the aquatic-terrestrial boundary presents a discrete hard edge between fragments. This can only be overcome in the adult phases of phytotelma-inhabiting species, for example, as winged phase of aquatic insects or after metamorphosis in tadpoles.

In addition to these hard edges, there can also be soft edges that act as secondary filters among separated phytotelmata. For instance, the physicochemical environment differs within each plant so that some hoverflies avoid bamboo internodes with low pH for oviposition (Kurihara, 1959) or mosquito larvae exhibit reduced survivorship with rising $\mathrm{pH}$ in tree holes (Carpenter, 1982). During extreme rainfall events, extensive flushing and recharging of the aquatic reservoir can occur and thus provide potential connectance among phytotelmata. The nutrient content (Carpenter, 1982) and pH (Clarke and Kitching, 1993) of phytotelmata can vary widely, and these varying levels of habitat restriction and fragmentation can create a 'hierarchy of fragmentation', with the imagines of phytotelm invertebrates being exposed to a less fragmented environment than the juvenile stages.

Phytotelmata fragmentation will have pronounced effects on the structure and function of ecological networks formed within such water bodies. Whilst there are examples of mutualistic interactions within pitcher plants (Clarke and Kitching, 1993), the vast majority of described phytotelma networks are antagonistic, and there is evidence for both bottom-up and top-down control within the food web (Hoekman et al., 2011; Kneitel and Miller, 2002). At least three discrete levels of fragmentation are apparent, from local to larger landscape scales (e.g. bromeliad leaf pools within a plant; phytotelmata within a single terrestrial matrix vs. multiple, fragmented terrestrial matrices). 
forces related to fluid viscosity or nutrient diffusion, whereas the herbivores that eat it will be more influenced by factors such as availability of physical refugia from predators (who in turn operate at larger scales), channel discharge or water depth (Woodward and Hildrew, 2002a; Woodward et al., 2010a). Thus, the fragment size within which each species operates tends to increase up the food chain, and the species' perception of edges also changes. In terms of 'flow habitats' in stream ecosystems, individual diatoms will be strongly influenced by boundary layer effects within the nearest few millimetres, herbivorous macroinvertebrates will respond to near-bed velocity and microhabitats at the scale of centimetres to metres, and predatory fish will respond to the availability of suitable territories at the pool-riffle or macrohabitat scale. The largest, most mobile, migratory species may even respond at the scale of the entire river catchment (Woodward and Hildrew, 2002a).

Most fragmentation studies usually focus on a particular spatial scale: Doak et al. (1992) reviewed 61 primary research papers on the effects of habitat fragmentation on population structure of terrestrial arthropods, all of which were conducted at a single spatial scale. In general, studies that account for fragmentation on different spatial scales are rare (but see Garcia and Chacoff, 2007; Schleuning et al., 2011b; Stephens et al., 2003). Forest fragmentation (large-scale reduction of fragment size) can affect ecosystem processes indirectly by changes in biodiversity, whereas selective logging (local scale) influenced ecosystem processes (e.g. pollination and seed dispersal) by modifying local environmental conditions and resource distributions (Schleuning et al., 2011b).

Many long-term consequences only become apparent after many decades (Laurance et al., 2011), yet most studies of anthropogenic fragmentation have been conducted over much shorter periods (Ewers and Didham, 2006), which may not be sufficient to detect the full range of responses. Nevertheless, empirical studies suggest that time lags in species responses at such time scales are very common (Ewers and Didham, 2006; Laurance et al., 2006b). While population densities may increase in the short term as survivors are concentrated in remaining patches, in the long term, species abundance and richness decline (Debinski and Holt, 2000) because some can survive for up to several generations under unsuitable habitat conditions before eventually going extinct ('extinction debt'; Tilman et al., 1994). Extinction debts can be pronounced if many species are near the threshold capacity of the landscape that ensures meta-population persistence (Hanski and Ovaskainen, 2000). Time-lagged responses of species to fragmentation are not only 
observed for long-lived trees, but also for other organism groups such as vertebrates and insects (Ewers and Didham, 2006; Metzger et al., 2009). Considering such time lags thus becomes especially important for evaluating fragmentation effects on species interactions and ecological networks.

Time lags are most pronounced where generation times strongly differ between interacting or dependant species (Kissling et al., 2008, 2010). For instance, in climate change impact assessments, low dispersal rates and long generation times of woody plants can slow distributional responses, with important consequences for bird species that depend on such plants for habitat and food (Kissling et al., 2010). In a fragmentation context, the different generation times of invertebrates and vertebrates, parasites and hosts, and species from different trophic levels in plant-animal mutualistic systems might lead to contrasting responses of interacting species, thus disrupting existing networks. For instance, long-lived vascular plants in European grasslands showed time-delayed extinctions whereas short-lived butterflies did not, even after 40 years (Krauss et al., 2010). This suggests that interacting species (at different trophic levels) have different extinction debts, so co-extinctions associated with long-lived taxa might amplify future biodiversity loss even without any further fragmentation occurring.

Given the various levels of complexity and spatiotemporal scales involved, a hierarchical approach seems necessary for understanding the effects of habitat fragmentation on species interactions, ecological networks and community-level changes (Didham et al., 2012; Urban et al., 1987).

\section{HABITAT FRAGMENTATION AND SPECIES TRAITS}

In addition to landscape attributes, species traits also modulate the effects of fragmentation (Aguilar et al., 2006; Ewers and Didham, 2006; Fahrig, 2003; Henle et al., 2004). For instance, overall species richness of butterflies in Europe and America decreases with fragmentation, but those with low dispersal ability, a narrow larval feeding niche and low reproduction are most strongly affected (Öckinger et al., 2010). In addition, intraspecific variation in phenotypic traits may ultimately affect community patterns, such as the distribution of niche width (Bolnick et al., 2011). In general, seemingly contradictory responses might be better explained by considering the role of species traits (Ewers and Didham, 2006). In this section, we briefly review fragmentation-relevant traits for plants and animals and then highlight the potential importance 
of species trait combinations for understanding the consequences of fragmentation for biodiversity and ecological networks.

\subsection{Plant traits}

Important plant traits for persistence in fragmented landscapes include seed dispersal, pollination and breeding system, growth form and seed bank (Table 1). Two aspects of seed dispersal are particularly relevant: dispersal mode and fruit traits (e.g. fruit and seed size). The former (abiotic dispersal by wind or via animal vectors) can strongly influence how the relative abundance of tree species responds to habitat fragmentation (Fægri and van der Pijl, 1979; Montoya et al., 2008; Tabarelli and Peres, 2002; Tabarelli et al., 1999). Additionally, fruit traits that influence frugivore choice (fruit size, edibility of the peel, defensive chemistry, crop size and phenology: Buckley et al., 2006) will influence the responses of fleshy-fruited plants to habitat fragmentation. Large, big-seeded fruits, which are consumed by only a few vertebrate species, might be most vulnerable to fragmentation (Corlett, 1998), and fruit size and colour may be crucial for plant colonisation of habitat fragments (Shanahan et al., 2001), where certain trait combinations attract a specific set of animal dispersers (e.g. birds vs. bats).

Plants also differ in their dependency on pollinators (e.g. Aizen and Feinsinger, 2003; Bond, 1994), and this can determine their vulnerability to fragmentation. Certain plants traits are especially important to attract pollinators and to exclude floral reward robbers, for example, flowering phenology, amount and quality of pollen and nectar, and structural complexity of the flower. Habitat fragmentation may contract flowering periods because abundant plant species should have longer populationlevel phenophases than rarer species (but see Morellato, 2004), increasing the risk of losing pollinators, which could further reduce plant fitness (Aizen and Feinsinger, 1994a,b).

Within species, flower morphology can vary among habitats: certain plants in urban fragments have more, but smaller flower heads, which may decrease floral attractiveness and affect pollinator behaviour (Andrieu et al., 2009). Changes in pollinator behaviour could increase self-pollen deposition (Aizen and Feinsinger, 1994a,b), and drive a divergence in the evolution of floral traits in fragmented populations (Kingsolver et al., 2001; Pérez-Barrales et al., 2007). Demographic, environmental and genetic stochasticity are likely to be most pronounced in small fragments (Matthies et al., 2004; Willi et al., 2005), and the latter may trigger a loss 
of self-compatibility alleles due to genetic drift, increasing inbreeding and genetic erosion (Ellstrand and Elam, 1993; Lande, 1988; Menges, 1991; Young et al., 1996; but see Aizen and Feinsinger, 1994a,b).

Differences in breeding systems can affect plant species responses to fragmentation. For instance, the herb Dianthus deltoides (Caryophyllaceae) is protandrous (i.e. anthers open before stigmas ripen) but in small fragments it becomes homogamous (i.e. the male and female sexual parts ripen simultaneously), increasing the probability of self-fertilisation (Jennersten, 1988). Self-compatible plants are often facultatively dependent on pollinators, whereas self-incompatible species are obligate outcrossers, relying exclusively on pollinators (e.g. Aguilar et al., 2006). Sex ratios in dioecious species might also be sensitive to fragmentation. In China, populations of the dioecious tree Pistacia chinensis (Anacardiaceae) were surveyed on islands of different size in a recently flooded reservoir ( $\mathrm{Yu}$ and $\mathrm{Lu}, 2011)$ : small islands with poor soils had a male-biased sex ratio, whereas large and nutrient-rich islands had a stable 1:1 ratio. Such drops in effective population size on small islands could accelerate population extinction.

\subsection{Animal traits}

The key animal traits in relation to fragmentation are dispersal ability, niche width, body size and sociality (Table 1 ), with the first two being especially important (Bommarco et al., 2010; Ewers and Didham, 2006). Species with high dispersal ability are less likely to be affected by fragmentation (Hanski and Ovaskainen, 2000; Öckinger and Smith, 2007; Roland and Taylor, 1997). For example, solely aquatic invertebrates must swim long distances if they are to colonise new streams in a river system, encountering many potential barriers to dispersal (Fig. 5), whereas larvae with winged adult phases can reach these new habitat fragments relatively easily. Although the abundance of adult phases of aquatic invertebrates (such as stoneflies) decreases exponentially with distance from their 'home stream', with the rate of decline varying with matrix permeability (Fig. 6), only a few gravid females may be needed to (re)populate an entire food web due to high-density-dependent predation on early life stages (e.g. Hildrew et al., 2004). This can lead to increased genetic differentiation in adult populations at larger distances between streams, highlighting the potential for genetic-level impacts of soft versus hard barriers to dispersal (Fig. 7).

Species with a wider dietary or habitat niche will also be less susceptible to fragmentation. Generalists may survive in very small patches by using 


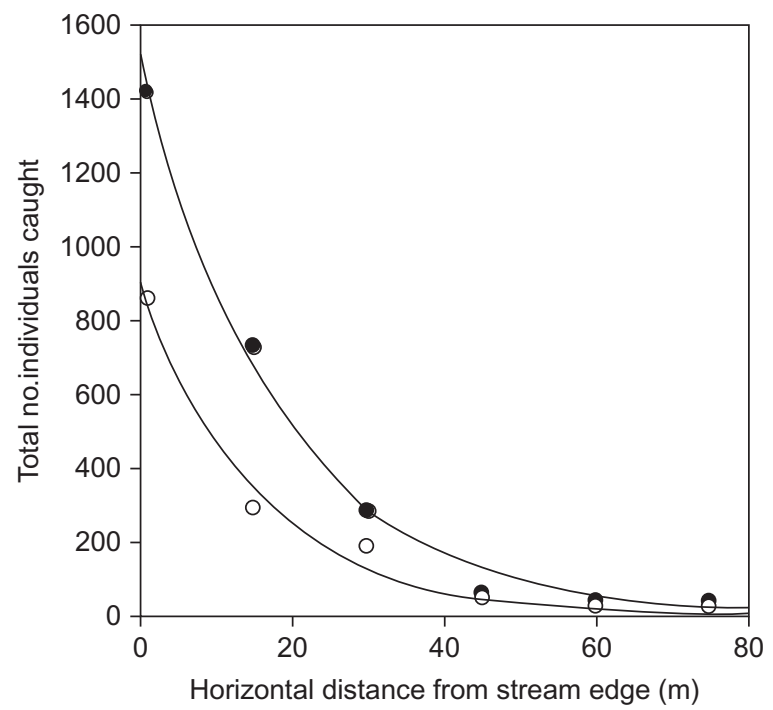

Figure 6 Lateral dispersal of winged adults of a common stonefly species (Leuctra nigra) from the stream edge through the terrestrial matrix (woodland-black circles; open land-white circles) within the Ashdown Forest, UK (see Fig. 16). Total number of males and females caught in passive Malaise traps are shown on the $y$-axis, with exponential declining models fitted for each habitat type [woodland: $y=1.517 \exp \left(-0.055^{*} x\right.$; $\left.R^{2}=0.99, \quad F=665.2, \quad p<0.001\right)$; open land: $y=903 \exp \left(-0.065^{*} x ; R^{2}=0.99\right.$, $F=324.6, p<0.001)]$. Redrawn after Petersen et al. (1999).

resources in both the fragment and the surrounding matrix (Andren, 1994). Specialists might find their resources (e.g. specific food plants) retained in only a few fragments, and habitat specialisation can further restrict their distribution. Some specialists also have a narrow geographic range (Gaston, 1988; Roy et al., 1998) again increasing the vulnerability to fragmentation. Finally, the trophic rank of a species is important and those at higher trophic levels are expected to be more sensitive because of their lower carrying capacity (Didham et al., 1996; Hance et al., 2007; Holt, 2002; Kruess and Tscharntke, 1994; Steffan-Dewenter and Tscharntke, 1999; SteffanDewenter, 2003; Tscharntke et al., 2002; Tylianakis et al., 2007; van Nouhuys, 2005, Vanbergen et al., 2006) and there is evidence from experimental food webs that this is indeed the case, although it is just one of several determinants (Ledger et al., 2012; Woodward et al., 2012).

Body size is a key trait as it determines home range size and dispersal ability for many species (Castle et al., 2011; Greenleaf et al., 2007; Haskell et al., 2002; Jetz et al., 2004; Leck, 1979; Lindstedt et al., 1986; Milton and May, 1976; Schaffer, 1981; Willis, 1979), and large species are often 


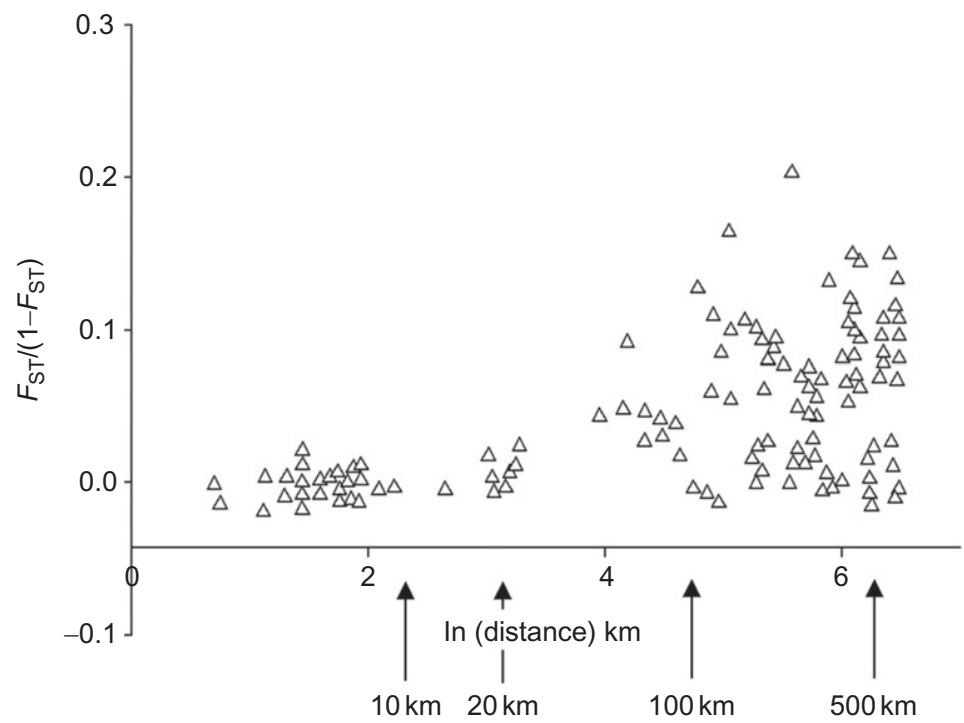

Figure 7 Genetic differentiation as an exponential function of geographical distance among 33 populations of a predatory caddisfly species (Plectrocnemia conspersa) across Britain, including 10 sites within the Ashdown Forest (Figs. 5, 6 and 16). Like all freshwater insects, this species has a larval aquatic phase and a winged terrestrial adult phase. The former are typically constrained to living in fragmented acid headwaters (where they are often top predators) within river networks, whereas the latter can disperse across land to connect otherwise isolated food webs. The genetic data above reveal panmictic populations at the regional catchment scale, with significant differentiation (measured as $F_{\mathrm{ST}} /\left(1-F_{\mathrm{ST}}\right)$ based on allozyme frequency data) occurring only at larger scales of fragmentation. Even though dispersal across large distances is a rare event (e.g. Fig. 6), only a few gravid females may be needed to repopulate an entire food web due to high fecundity combined with strong density-dependent mortality early in the life cycle (Hildrew, 2009; Hildrew et al., 2004). Redrawn after Wilcock et al. (2003).

especially vulnerable - unless they are able to span the gaps between fragments (Crooks, 2002; Ewers and Didham, 2006; but see Laurance et al., 2011). In Amazonia, wide-ranging forest bird species (van Houtan et al., 2007) and primates (Boyle and Smith, 2010) are more vulnerable to fragmentation than those with smaller territoria, and species with limited spatial requirements such as small mammals, non-trap-lining hummingbirds and ants are generally less susceptible (Laurance et al., 2011). Besides body size, restricted mobility, resource specialisation, low annual survival rate, high population variability, and terrestrial foraging and nesting increase vulnerability among birds to fragmentation (Sieving and Karr, 1997). 
Species that are large and/or rare are especially vulnerable to the effects of habitat fragmentation by drought in stream food webs (Ledger et al., 2012).

In bees (Box 4), relationships between habitat loss and species traits have been intensively studied (Krauss et al., 2009; Moretti et al., 2009; SteffanDewenter et al., 2006), with diet width and sociality being especially important (Aizen and Feinsinger, 1994a,b; Klein et al., 2003; Öckinger and Smith, 2007; Rundlöf et al., 2008; Steffan-Dewenter et al., 2002). Social bees are expected to outperform solitary taxa in harvesting resources because of their higher foraging and food-provision capacity (e.g. Bommarco et al., 2010) and communication systems (e.g. the waggle-dance in honeybees). Social bee species are always diet generalists, because their long-lasting colony needs food throughout the year, although there are differences between tropical and temperate areas. In temperate regions, wild social bees (Bombus spp.) appear to be less sensitive to habitat fragmentation than solitary bees (Steffan-Dewenter et al., 2002), whereas in the tropics solitary bees appear to be less sensitive to land-use change than social stingless bees (Aizen and Feinsinger, 1994a,b), probably due to their specialisation on forest as nesting habitat (Roubik, 1989, 2006). In bumblebees (Bombus spp.), long-tongued species have declined more than short-tongued ones due to changes in agricultural practices and habitat fragmentation (Bommarco et al., 2012; Dupont et al., 2011), and late-season species have declined more than early-season species (Fitzpatrick et al., 2007).

\subsection{Species trait combinations}

Any given species comprise a suite of traits, some of which are strongly correlated, whereas others may be orthogonal (Herrera, 2009). Data on individual traits of species, however, are insufficient for predicting fragmentation effects on biodiversity (Ewers and Didham, 2006): rather, their combination and the wider ecological context are both key here. For more detailed examples, see the textboxes on bees (Box 4) and avian frugivores (Box 5).

A combination of body size, diet, dispersal ability, habitat specialisation and sociality may be needed to predict species responses to fragmentation (Boyle and Smith, 2010; Milton and May, 1976). For instance, among European bees, large dietary generalists are less affected by fragment area than small generalists, whereas small specialists may be less affected than large specialists (Bommarco et al., 2010). In Amazonian forest 


\section{BOX 4 Bees as Network Nodes}

Bee species vary widely in body size, foraging mode, social organisation, seasonal activity and specialisation on flower resources. These characteristics play an important role for the structure and dynamics of plant-pollinator networks. Large body size reflects the capacity to fly longer distances, and genetic markers and radio-tracking techniques are increasingly used to estimate actual flight distances (Darvill et al., 2004; Hagen et al., 2011). Small stingless bees (e.g. Plebeia and Tetragonisca) often fly distances of up to $1 \mathrm{~km}$ while flight distances for larger Melipona species can be $>2 \mathrm{~km}$ (Araújo et al., 2004). The largest orchid bees (Eulaema; $18-31 \mathrm{~mm}$ ) can fly up to $23 \mathrm{~km}$, including the crossing of unsuitable matrix habitat such as open waters for several kilometres (Janzen, 1971; Wikelski et al., 2010). In this bee group, patrolling flights of males can reach long distances and thus disperse pollen among fragmented plant populations. Large bees (e.g. Xylocopa, Bombus, Centris, Epicharis, Eulaema and Oxaea) are hence particularly important for connecting habitat fragments. However, bee mobility also depends on the abundance of food resources and on the amount of floral awards.

Sociality and behavioural differences also affect bee species responses to habitat fragmentation. Not all are as highly eusocial as the honeybees (Apis spp.) and the stingless bees (Meliponini): most species are solitary (the female performs all tasks) or subsocial and semisocial (some cooperation among the females). These differences in social organisation can strongly influence network topology due to differences in the abundance of individuals in the nest (one, few, hundreds or thousands). Most eusocial species have perennial colonies, tend to be floral resource generalists and need resources throughout the year, at least in the Tropics, and these tend to be key species or hubs in ecological networks. Most bee species in the tropics are also multivoltine (multiple generations per year), and some (e.g. Xylocopa) are long-lived, which can affect the temporal dynamics of plant-pollinator interactions due to differences in abundance and phenophase length. In arctic or temperate regions, where climatic seasonality is pronounced and univoltine bee species are dominant, temporal dynamics in the structure of plant-pollinator networks have already been empirically demonstrated (Olesen et al., 2008).

Resource specialisation also influences network structure (e.g. the range of available nectar plants is broader than that of pollen plants) because nectar is mainly consumed by the adults whereas pollen is used in the brood cell to feed the larvae (Cane and Sipes, 2006). In general, oligolectic bees are recognised for their specialised floral niches whereas polylectic bees (e.g. social species such as Apis, Bombus and Meliponini) visit a wide range of plants (including flowers of different morphology, colour, size, etc.): within the interaction network, the latter species represent highly connected nodes. 


\section{BOX 5 Avian Frugivores and Seed Dispersal in a Fragmented World}

Avian frugivores predominate in warm and wet climates of the world's tropical and subtropical regions (Fleming et al., 1987; Kissling et al., 2009). Of the $>1200$ frugivorous bird species worldwide, most $(\sim 50 \%)$ are found within the order Passeriformes (perching birds) (Kissling et al., 2009, 2012b), with a body mass of usually $<200 \mathrm{~g}$. Over 100 species of frugivores are also found in the orders Columbiformes (doves and pigeons), Psittaciformes (parrots) and Piciformes (woodpeckers, toucans, barbets, honeyguides) (Kissling et al., 2009, 2012b). The spatial distribution patterns vary among bird orders with frugivorous perching birds and parrots dominating in the Neotropics and frugivorous pigeons and hornbills prevailing in Southeast Asia (Kissling et al., 2009). Given the spatial heterogeneity of future land-use changes on bird distributions (Jetz et al., 2007) and the taxonomic and geographic differences in frugivores among regions (Kissling et al., 2009), the global consequences of habitat fragmentation for seed dispersal of fleshy-fruited plants are likely to be complex.

At the landscape scale, the effectiveness of seed dispersers is characterised by the quantity and quality of seed dispersal (Schupp et al., 2010), which in turn is dependent upon body size and associated life history behavioural traits. Due to their requirements for extensive home ranges, large frugivorous birds are especially extinction prone in small fragments (Renjifo, 1999; Uriarte et al., 2011). The ability to fly long distances allows large-bodied frugivores to connect habitat patches (Lees and Peres, 2009; Spiegel and Nathan, 2007). Habitat fragmentation can cause changes in the movement patterns of frugivores, with consequences for seed dispersal (Lenz et al., 2011), especially for plants with large, big-seeded fruits because their dispersal often only depends on one or a few large frugivores (Corlett, 1998; Guimarães et al., 2008). Seed dispersal effectiveness of plants with smaller fruit largely depends on the range of frugivore body sizes in the network, with smaller frugivores allowing for within-patch dispersal and larger frugivores for between-patch dispersal (Spiegel and Nathan, 2007). In addition to body size per se, gut retention times and movement velocities of frugivores also determine seed-dispersal distances (Schurr et al., 2009). The interplay of animal behaviour, plant and animal traits, and the specific characteristics of the landscape thus produce complex seed dispersal kernels (Morales and Carlo, 2006) and seed dispersal effectiveness landscapes (Schupp et al., 2010).

fragments, the most capable gap-crossers among birds are medium or large species of insectivores, frugivores and granivores, and these species dominate in small patches (Lees and Peres, 2009). Certain species trait combinations can amplify (or mitigate) vulnerability to fragmentation. For instance, on Barro Colorado Island (Panama), the largest bird was 
the Black-faced Antthrush (Formicarius analis), which also had low annual recruitment and survival rate, and this potent combination of traits, which are often combined in many other species, could explain why it went extinct particularly rapidly as its habitat fragmented (Sieving and Karr, 1997).

Trait matching between interacting plants and animals could affect higher-level responses to fragmentation. For instance, interactions in some plant-pollinator networks show size matching, that is, insect species with a long proboscis visit a wider range of flowers than do species with a short proboscis (e.g. Borrell, 2005; Corbet, 2000; Goldblatt and Manning, 2000; Harder, 1985; Stang et al., 2009).

Developing a combined trait-response framework could provide important future advances in assessing fragmentation effects in ecological networks. Additionally, interaction effects between fragment characteristics (see Section 3.2) and species traits could also be important. Network analysis offers a potentially powerful way to identify modules of species with similar responses to fragmentation, which then may be analysed with respect to their trait combinations (Verdú and Valiente-Banuet, 2011).

\section{HABITAT FRAGMENTATION AND BIOTIC INTERACTIONS}

In the previous sections, we have examined the importance of landscape structure (e.g. fragment characteristics, habitat edges, matrix) and species traits for assessing the consequences of habitat fragmentation on biodiversity: here, we turn our attention to impacts on species interactions, the strengths and outcomes of which (Fig. 2) vary spatially and over time. This spatial dependency arises because the probability of an encounter between predator and prey, pathogen and host, or mutualistic animals and their plants has a landscape context, and hence sensitivity to fragmentation.

\subsection{Mutualistic plant-pollinator interactions}

Pollination and, hence, plant reproduction can be strongly affected by habitat loss and fragmentation (Fægri and van der Pijl, 1979; Jennersten, 1988; Kearns et al., 1998; Olesen and Jain, 1994; Rathcke and Jules, 1993; Renner, 1998) (for examples see also Box 4).

Due to habitat fragmentation, pollinator communities could become more homogenous, and generalists (Ewers and Didham, 2006) and introduced species (e.g. Do Carmo et al., 2004) may replace natives and dominate 
interactions, potentially altering the reproductive output of the plant community. However, the effect on pollen dispersal and pollination effectiveness may strongly vary among species, without necessarily being related to a species habitat niche: habitat generalists and invasive pollinators can either be less (Didham et al., 1996; Do Carmo et al., 2004) or, in some cases, more effective pollinators than habitat specialists (Dick, 2001).

Fragmentation can isolate host plant patches, reducing genetic and ecological exchange among them. Although still little is known about precise flight distances and movement patterns of pollinators at the landscape scale (Hagen et al., 2011), body size influences the genetic connectance of, and pollen flow among, distant plant populations (Pasquet et al., 2008). The effective movement of pollinators may be tracked by paternity assignment of seeds and pollen (Lander et al., 2011), and the influence of landscape configuration on pollinator movement (e.g. for trap-lining species) can be incorporated into the analysis (Lander et al., 2011).

The reproductive output of plants can vary with pollinator composition, abundance and behaviour (Lamont and Barker, 1988; Lamont et al., 1993). Although visitation rates are expected to be influenced by habitat fragmentation, the results are inconclusive: some pollinators are more abundant in larger fragments (Sih and Baltus, 1987), some are equally abundant (Jennersten, 1988), while others are rarer in fragments (Sih and Baltus, 1987; Strickler, 1979). Temporal aspects such as phenological changes influence how fragmentation affects plant-pollinator interactions (Memmott et al., 2007). When fragmentation reduces plant species richness, food shortages could reduce pollinator diversity, especially among long-living insects, such as bumblebees (Memmott et al., 2007).

The local extinction of pollinators might not always have consequences for interacting plants, if redundant species can compensate. For instance, the Hawaiian tree Freycinetia arborea (Pandanaceae) was once pollinated by now extinct birds, but has recently been rescued from extinction by an introduced white-eye bird (Zosterops sp.) that replaces previous pollinator species (Cox, 1983). If redundancy is not evident, even the loss of single interactions can initiate waves of further extinctions (Nilsson et al., 1992; Olesen and Jain, 1994). For instance, the orchid Cynorkis uniflora is a mountain rock plant highly specialised upon a few pollinating sphingids in Madagascar (Nilsson et al., 1992). The host plants of the larvae of these sphingids are found in nearby forests, and the delicate orchid-pollinator adult/larva interactions are highly vulnerable to forest loss and fragmentation. The extinction of 
the pollinating hawkmoths can trigger the loss of orchids and initiate a 'cascade of linked extinctions' (Myers, 1986).

\subsection{Mutualistic plant-frugivore interactions}

Mutualistic interactions between fleshy-fruited plants and frugivores play a central role for assessing the consequences of habitat fragmentation on biodiversity, especially in the Tropics (Box 5). Frugivorous vertebrates are the focal seed dispersers because only very few invertebrates (e.g. ants, earthworms and grasshoppers) play this role (Duthie et al., 2006; Rico-Gray and Oliveira, 2007; Willems and Huijsmans, 1994).

Larger animal species are expected to be particularly sensitive to habitat fragmentation (Haskell et al., 2002), and there is supporting evidence of this for frugivorous birds (e.g. Sub-Andeans: Renjifo, 1999; Amazonia: Uriarte et al., 2011). The proportion of fruit in primate diets is positively correlated with home range size (Milton and May, 1976) and species persistence in forest fragments (Boyle and Smith, 2010). The disappearance of large frugivores thus decreases the probability of long-distance dispersal of fleshy-fruited plants from small patches and fragments (Fragoso, 1997; Fragoso et al., 2003; Spiegel and Nathan, 2007; Uriarte et al., 2011). The response of small-to-medium-sized frugivores to fragmentation is probably driven by species' habitat specialisation and matrix tolerance, and their ability of gap-crossing (Table 1). Compared to medium-sized frugivores, mesopredators (i.e. medium-sized carnivorous habitat generalists) move more freely between matrix and fragment (Terborgh et al., 1997).

The traits of fleshy-fruited plants determine frugivore choice and hence endozoochorous seed dispersal and the relationship between fruit size, consumer size and gape width is the key (Buckley et al., 2006; Burns and Lake, 2009; Jordano, 1995; Lord, 2004). Small fruits are typically consumed by a wide range of potential seed dispersers, including many species that thrive in small forest fragments and degraded landscapes (Corlett, 1998). However, large, big-seeded fruits tend to have fewer dispersers, and the very largest may depend on only one or a few species (Corlett, 1998). Consequently, these species are the specialists in the network and most vulnerable to fragmentation. More generally, the proportion of fleshy-fruited species is likely to decrease in smaller fragments (Tabarelli and Peres, 2002).

Beyond fruit size, the presence of an inedible pulp, defensive chemicals, crop size, fruit colour and fruiting phenology also influence frugivore choice (Buckley et al., 2006; Voigt et al., 2004; Willson and Whelan, 1990), but if 
and how they relate to fragmentation is currently unclear. Pre-and postingestion processing of fruit and movement of consumers determine seed-dispersal distances and plant establishment patterns (Buckley et al., 2006; Schurr et al., 2009; Spiegel and Nathan, 2007). The mean dispersal distance of endozoochorously dispersed seeds depends upon a combination of frugivore body size, mobility and gut retention time (Schurr et al., 2009). Large frugivores (e.g. the trumpeter hornbill Bycanistes bucinator) may change their movement patterns, with unimodal seed-dispersal distribution within forests but bimodal distribution in fragmented agricultural landscapes (Lenz et al., 2011). Individual fruiting trees, even exotic ones, in farmland may be important food sources for the frugivore community and thus represent foci for seed dispersal and forest regeneration, even in highly degraded landscapes (Berens et al., 2008; Fisher et al., 2010).

\subsection{Mutualistic plant-ant interactions}

Another type of mutualism that is important in a fragmentation context is the interaction between ants and plants in defensive mutualist systems (Box 6).

The intimacy of this interaction (i.e. the degree of biological association between individuals of interacting species) varies, and this could determine how plant-ant interactions respond to habitat fragmentation. Some plant-ant defensive mutualisms, such as extrafloral nectary-based mutualisms, are typical among free-living species (Guimarães et al., 2007), that is, each individual ant and plant can interact with dozens of partners from different species through its lifespan. These are therefore similar to most of the pollination and seed dispersal interactions with respect to degree of interaction intimacy (Guimarães et al., 2007). In contrast, many plant-ant mutualisms are symbiotic, that is, one individual plant hosts an ant colony and, as a consequence, individuals (the plant and the ant colony in this case) interact with one or a few partners through their lifetime (Fonseca and Benson, 2003; Fonseca and Ganade, 1996). Few studies have investigated how environmental change affects the network structure of plant-ant interactions (Diaz-Castelazo et al., 2010), but information about these mutualistic interactions is becoming increasingly available.

Key traits in extrafloral nectary interactions include ant body size (Chamberlain and Holland, 2009) and the distribution of ant and/or plant abundances (Chamberlain et al., 2010), which are likely to change with 


\section{BOX 6 Interactions Between Ants and Plants}

Ants form one of the dominant groups in terrestrial ecosystems, and they interact in multiple ways with plants (Rico-Gray and Oliveira, 2007), as seed predators (Rico-Gray and Oliveira, 2007), leaf-harvesters (Oliveira et al., 1995; Pizo and Oliveira, 2000) and mutualistic partners (Christianini and Oliveira, 2009; Palmer et al., 2008; Rico-Gray and Oliveira, 2007). Although ant pollination is rare (Beattie, 1985; Gómez, 2000), ants are among the main seed dispersers of many plant species (Culver and Beattie, 1978; Pizo et al., 2005). In some tropical ecosystems, ants form gardens (Davidson, 1988), actively dispersing seeds of plants and nesting within the plant parts. Finally, ants are among the most conspicuous defensive mutualists of plants (Rico-Gray and Oliveira, 2007), which offer extrafloral nectar, other food resources and/or nesting sites such as domatia.

Recent studies of extrafloral nectary assemblages suggest ant body size and species abundance are important in shaping patterns of interactions: the number of interactions increases with ant body size (Chamberlain and Holland, 2009; Chamberlain et al., 2010). These results mirror those often reported in predator-prey interactions (Sinclair et al., 2003) and plant-frugivore mutualisms (Jordano, 2000). Several hypotheses suggest that the effects of ant body size are more indirect than direct, with larger ants interacting with more plant species than smaller ants because they (i) forage over a greater area, (ii) are more widely distributed or (iii) because of size-driven competition hierarchies (Chamberlain and Holland, 2009). In the latter scenario, larger ants, that often recruit fewer workers when foraging, are outcompeted by smaller recruitmentefficient ant species from the optimal resources, leading to an increase in the number of plants the larger ants interact with.

habitat fragmentation. The effects of fragmentation can differ among ant functional groups (Pacheco et al., 2009; Wirth et al., 2008), and it may even benefit some plant-ant networks, which often naturally occur in habitat edges (e.g. Cecropia spp and its ant partners). Predicting which ant or plant species will be affected, and how, requires an understanding of the underlying traits shaping these interactions. The challenge is that we still need to improve the taxonomy of a considerable fraction of ant species, and the natural history of many species still remains unknown. In this context, the phylogenetic relatedness of interacting species is a proxy for non-random trait distributions.

Understanding the ecological and evolutionary dynamics in these complex fragmented landscapes faces challenges similar to other kinds of 
interaction. For example, plant-ant interactions involve organisms that differ radically in how they perceive their environment. Ants are small, short-lived organisms, whereas plants are much larger and often longer-lived. Thus, they will perceive the effects of habitat fragmentation at distinct scales and will respond in different ways. Additionally, all plants and most ant colonies are essentially fixed in space, whereas most other plant-animal mutualisms involve a fixed individual (e.g. plant) and a mobile forager (e.g. pollinator). Thus, dispersal of both ants and plants is a between-generation process, which may lead to as yet unexplored meta-community dynamics that differ from other types of network. Moreover, plant-ant protective mutualisms are based on indirect benefits: plants benefit from a trophic cascade caused by ants attacking herbivores (Perfecto and Vandermeer, 2008; Vandermeer et al., 2010). Thus, if habitat fragmentation changes the intensity of herbivory, it also changes the fitness consequences of the mutualism (see Palmer et al., 2008).

\subsection{Antagonistic interactions within food webs}

While the previous sections have focused on mutualistic interactions, we now address antagonistic interactions, specifically food webs. Body size is a key determinant of predator-prey interactions in many food webs (Emmerson and Raffaelli, 2004; Woodward et al., 2005), with large predators typically consuming smaller resources (Layer et al., 2010; McLaughlin et al., 2010), especially in aquatic systems. As a result, trophic height tends to increase with body mass (Jonsson et al., 2005; O'Gorman and Emmerson, 2010), although predator-prey body mass ratios may decline (Brose et al., 2006; Jonsson and Ebenman, 1998; Mulder et al., 2011). Since large species are most susceptible to habitat fragmentation due to their perception and use of resources over larger distances (Holt, 1996) and their need for larger home ranges (Haskell et al., 2002), top predators should be especially prone to extinction. As they often exert strong effects within food webs, their loss could have severe implications for network structure and stability, although recent field experiments suggest that this might be primarily via direct effects of their loss from the system rather than more subtle indirect food web effects per se (Woodward et al., 2012).

Habitat fragmentation can reduce encounter rates and hence interaction strengths within food webs. This may ultimately decouple pairwise interactions, leading to a simpler and potentially more fragmented food web, since the starting point at which a food web assembles is the level of interactions among individuals. In many food webs, predators (and other non-predatory 
consumers) are often far from satiation as indicated by the high proportions of relatively empty predator guts compared with what they could consume if feeding rates were maximal (Woodward and Hildrew, 2002b). This suggests that encounter rate is a key determinant of the strength of predator-prey interactions and network structure (Petchey et al., 2010; Woodward et al., 2010b).

In a fragmented landscape, encounter rate can be influenced at different spatial and temporal scales, from short-term patch-scale aggregative responses of predators to their prey within particular fragments during disturbance events (e.g. Lancaster, 1996) to larger scale habitat-level effects that reflect longer-term depletion of prey by predators.

Handling time is also important for food web structure and dynamics, but it is difficult to envisage how it might be affected by fragmentation, as it seems likely to be relatively robust to this kind of disturbance (e.g. in contrast to the effect of temperature changes). Thus, encounter rate rather than handling time might change under increasing levels of fragmentation, and the relative importance of the two rates could be key for predicting the higher-level effects in food webs (e.g. Petchey et al., 2010).

As in mutualistic networks, the scale and environmental grain of fragmentation will also interact with species life histories to determine food web effects. For instance, in fresh waters undergoing fragmentation (e.g. temporary pools formed by the retreat or drying of waters from floodplains), food web interactions can be intensified in the short (i.e. intragenerational) term if predators and prey are concentrated in increasingly smaller patches. Conversely, fragmentation may weaken top-down effects in the longer (intergenerational) term if large predators are lost from small habitat patches. Here, meta-population and source-sink dynamics and the ability of predators and prey to recolonise isolated or small habitat patches may be key, and species traits such as body size, behaviour, life history and taxonomic identity will influence these dynamics (Ledger et al., 2012).

\subsection{Antagonistic host-parasitoid interactions}

Antagonistic host-parasitoid interactions can also be affected by habitat fragmentation, and the degree of specialisation of parasitoids on their host is likely to be critical aspect here. When the host is restricted to certain plant species or habitats, highly host-specific parasitoids will experience landscapes as islands within a sea of unusable matrix. Conversely, for a more generalist parasitoid, capable of using hosts from different habitats, the landscape 
represents a mosaic of variable-quality patches. Fragmentation should therefore have increasingly negative effects on more specialised parasitoids, and several empirical studies support this conclusion (moth parasitoids: Elzinga et al., 2007; aphid parasitoids: Rand and Tscharntke, 2007; leafminer parasitoids: Cagnolo et al., 2009; parasitoids of cavity-nesting bees and wasps: Holzschuh et al., 2010). These findings suggest that the effects of fragmentation on parasitoids will largely be mediated by altered host distributions, which are often coupled to plant densities (for herbivorous hosts) at the patch scale (Albrecht et al., 2007; Amarasekare, 2000; Cronin et al., 2004; Holzschuh et al., 2010; Kruess, 2003; Schnitzler et al., 2011; Vanbergen et al., 2007).

Although within-patch effects may be important in determining parasitoid densities, the location of refuge habitats, parasitoid attack rates and dispersal ability will determine parasitoid-host dynamics at a landscape scale (Mistro et al., 2009). The survival of a parasitoid meta-population will thus largely depend on individual dispersal abilities, and body size constraints might be important here (Roland and Taylor, 1997). Furthermore, dispersal limitation may moderate parasitoid-host interactions (Thies et al., 2005) because higher trophic levels are likely to be most negatively affected by fragmentation (Holt, 1997). The species-specific extent of dispersal limitation could ultimately determine the relative competitive success of different parasitoid species and how they experience the host landscape (van Nouhuys and Hanski, 2002). As a consequence of habitat fragmentation, attack and parasitism rates can change depending on the fragment isolation, matrix quality and the amount of suitable habitat in the landscape (Cronin, 2003; Kruess and Tscharntke, 2000; Roland and Taylor, 1997). The combination of within-patch effects (habitat quality, host abundance), landscape characteristics (fragment characteristics) and species traits (e.g. dispersal ability and body size) can thus ultimately produce a variety of outcomes for parasitoid-host interactions.

\subsection{Summary of fragmentation effects on mutualistic and antagonistic interactions}

The responses of biotic interactions to habitat fragmentation are complex, but several key themes arise repeatedly for both mutualistic and antagonistic interactions. The core question is how habitat fragmentation (e.g. fragment size and isolation) will change the links between species, and these are, in 
turn, a product of the functional traits of the interacting species (e.g. body size, dispersal ability, level of specialisation).

A key species trait is body size because it affects how species interact and their responses to habitat fragmentation. Its importance is evident in plant-pollinator interactions (e.g. proboscis length and size of floral structures), plant-frugivore interactions (e.g. gape width and fruit sizes), plant-ant interactions (e.g. size-driven competition hierarchies) and food webs (e.g. predator-prey mass ratios). Beyond general effects of body size and trophic rank on species interactions, the size of an animal (or plant) also correlates with a suite of other fragmentation-relevant traits. In particular, body size determines dispersal ability and movement distances of some taxa, a fundamental aspect for persistence in a fragmented landscape. Body size measures are often used as proxies for estimating movement distances indirectly, including body mass for birds and mammals (Haskell et al., 2002; Jetz et al., 2004), measures of wing shape in birds (Dawideit et al., 2009), and body length, intertegular span or wing span for insects (Cane, 1987; Greenleaf et al., 2007; Michener, 2007; Rogers et al., 1976). Similarly, fruit sizes can be used as a proxy for long-distance dispersal in fleshy-fruited plants, at least when body sizes of their extant vertebrate dispersers are correlated with seed dispersal effectiveness (sensu Schupp et al., 2010). Given the tremendous differences in body sizes among species involved in interactions (e.g. insects vs. vertebrates), responses of different-sized mutualists and antagonists should vary markedly even within the same level of fragmentation.

Specialisation also influences how fragmentation affects mutualistic and antagonistic interactions. The degree of habitat specialisation (e.g. forest dependence or matrix tolerance) is important because mutualistic and antagonistic interactions will change, as specialised species are lost as fragmentation proceeds. Dietary specialisation is particularly important in antagonistic interactions, but also in many mutualistic interactions. In this context, trophic redundancy may be key to buffering species losses. For instance, in mutualistic interactions, the functional loss of a species may be compensated by another species of similar size (cf. Zamora, 2000). As body-size distributions are typically skewed towards small species (Woodward et al., 2005), the potential for functional redundancy decreases with increasing body size (and trophic status). Consequently, large species may be functionally more important for conserving size-dependent ecosystem services, that is, seed dispersal and pollination in mutualistic networks, pest control by predators and biomass production for human consumption in fisheries (Rossberg, 2012). 
A couple of other aspects, such as the role of animal behaviour, emerge as important drivers of how fragmentation will affect biotic interactions, but they might be specific to a particular interaction type. In plant-frugivore interactions, movement behaviour and gut retention times of frugivores will influence seed dispersal kernels at the landscape scale (Box 5). Flower and fruit handling behaviour are strongly species-specific and will alter pollination and seed dispersal effectiveness in mutualistic networks. Furthermore, differences in sociality (e.g. solitary vs. social bees) will influence spatiotemporal abundances of individuals and resource specialisation. To some extent, such behaviours are phylogenetically conserved, so taxonomic identity can provide important information in this regard. Unfortunately, in many instances, we still know little about the natural histories of interacting species and the importance of link strength, especially in tropical regions, which at present constrains our ability to generalise about fragmentation effects on mutualistic and antagonistic interactions.

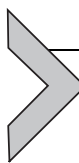

\section{EFFECTS OF HABITAT FRAGMENTATION ON DIFFERENT KINDS OF NETWORKS}

\subsection{General introduction}

Habitat fragmentation influences biodiversity at different organisational levels, from individuals to species populations, communities and multispecies ecological networks (e.g. Didham et al., 1996; Hill et al., 2011; Krauss et al., 2010). To date, little is known about how ecological networks of interacting individuals and species change in response to habitat fragmentation. Here, we address potential consequences for the structure of mutualistic and antagonistic networks (rather than only interactions per se, see Section 5).

\subsection{Mutualistic plant-pollinator networks}

Pollination networks are the most species-rich of all mutualistic networks, globally involving $88 \%$ of all angiosperm species, at least 1 million insect species belonging to several orders, about 1000 species of birds, hundreds of lizards and perhaps more than 100 mammals (Carstensen and Olesen, 2009; Olesen and Valido, 2003; Ollerton et al., 2011; Box 7). This translates into a rich functional diversity with respect to body size, morphology, mobility, behaviour and breeding systems, which further leads to a wide variety of adaptive strategies for locating, accessing and exploiting resources. These strategies vary in space and phenotypic 
plasticity, further complicating our efforts to predict outcomes of ongoing habitat fragmentation processes.

First, habitat fragmentation reduces overall species abundance in pollination networks and then later species and link richness (e.g. Aizen et al., 2008; Morales and Aizen, 2006), for example, butterfly species richness and

\section{BOX 7 Diversity and Mobility of Pollinators}

Pollinators are known from a wide variety of invertebrate and vertebrate taxa, but not all groups are equally represented in all networks. There is considerable spatial variation, for example, bird pollination is rare on mainland in Europe whereas it is common on European islands (Dupont et al., 2004; Kraemer and Schmitt, 1999; Olesen, 1985; Olesen and Valido, 2003; Ollerton et al., 2003). Similarly, bat pollination is common in the Tropics but virtually unknown from temperate or arctic regions (Proctor et al., 1996; however, see Ecroyd, 1993). The taxonomic diversity of flower-visiting animals translates into a broad range of species traits. For example, body size may vary up to 2000 -fold, from tiny insects (e.g. wasps with a body length of $0.2 \mathrm{~mm}$ ) to large mammals (e.g. flying foxes, up to $400 \mathrm{~mm}$ in body length), while body size in plant-frugivore networks may typically vary over one or two orders of magnitude between small birds and mammals (Fleming et al., 1987). The high diversity of pollinators results in different strategies for accessing and exploiting floral resources and in a high variability of how species respond to environmental disturbances (Kearns, 2001). For instance, flies show very complex and varied life histories, with larval habitats ranging from predatory through saprophytic and parasitic. In contrast, bees rely on floral resources during all their life stages (Michener, 2007). Thus, in flies, larval food supply might be more important for responses to habitat fragmentation than flower availability to the adult forms (Bankowska, 1980).

Foraging distances of pollinators range from a few metres to several kilometres (excluding migration), and almost all taxonomic groups contain sedentary as well as highly mobile species. For insects, which comprise the largest and most diverse group of pollinators, large amplitudes of foraging ranges have been reported: small solitary bees may fly only a few hundred metres whereas larger species can fly $10-20 \mathrm{~km}$ (Box 4). Much less is known about space use and foraging ranges of other pollinator groups, although in syrphid flies, a species-rich group of important flower-visitors, a few species may migrate over hundreds of kilometres (Torp, 1994), while resident species tend to stay within a very limited area. Beetles, a relatively minor group among pollinators, tend to be sedentary and less mobile than other groups (Proctor et al., 1996). Butterflies can be classified into three mobility classes: sedentary, intermediately mobile 


\section{BOX 7 Diversity and Mobility of Pollinators-cont'd}

and migrant species. While migrants may disperse hundreds to thousands of kilometres, sedentary species are very local, often limited to one patch of food plants (Pollard and Yates, 1993). A small group of flower-visitors are the lizards, which appear to be important for pollination on islands (Olesen and Valido, 2003). Little is known about their foraging ranges (Nyhagen et al., 2001), but for the common and widespread, generalist flower-visiting endemic gecko Phelsuma ornata in Mauritius, $89 \%$ of marked individuals were re-sighted on the next day less than $15 \mathrm{~m}$ from the place of release, while maximum dispersal range was $<90 \mathrm{~m}$ (Nyhagen et al., 2001). The foraging range of nectarivorous birds depends both on body size and behaviour (Craig et al., 1981; Gill and Wolf, 1975). For hummingbirds, these interconnected attributes can translate into different community roles (Feinsinger, 1978). For instance, some species are trap-liners tracking spatially dispersed flower resources in a repeated route whereas other species are territorial and defend clumped resources, highlighting the potential for behavioural traits to determine the network consequences of fragmentation (Laurance, 2004).

composition per fragment decline with fragmentation (Öckinger et al., 2010). This process is called network contraction (Fig. 8; Valladares et al., 2012).

In pollination networks, abundance of species is positively correlated to their linkage level (Fig. 9; Olesen et al., 2008; Stang et al., 2009). During fragmentation, some pollination systems may disappear completely as abundance declines (Girao et al., 2007). In pollination networks, plants are generally longer-lived than their pollinators, resulting in an accumulation of time-delayed plant extinctions (Krauss et al., 2010). Thus, rare specialist pollinators (linkage level $\leq 2$ links to other species), which constitute about half of all pollinator species in networks, are the first to go (Olesen, 2000). However, fewer pollinator species in a network does not necessarily compromise the fecundity of all plants, because the outcome depends also on the effectiveness of the pollinators (Perfectti et al., 2009). It can even be beneficial if the most abundant pollinators are the most effective, because other pollinators, which might be less efficient or less specialised pollinators or even nectar and pollen robbers, disappear (Genini et al., 2010). However, according to a supposed positive complexity-stability relationship, fewer species and links in pollination networks lower their disturbance resilience (e.g. Okuyama and Holland, 2008). 
Plants sorted from left to right according to increasing nectar tube length $n$

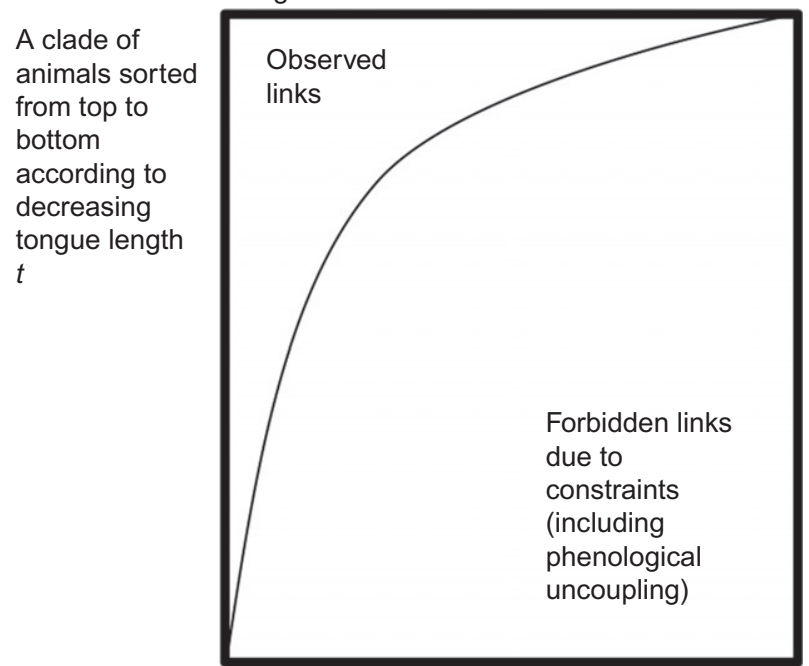

Figure 8 Pollination network contraction. During habitat fragmentation, pollinator specialists at the bottom left of the interaction matrix and plant specialists at the top right go extinct because of their low abundance. The first links to go extinct lie in concave bands running between lower left and upper right corners. Consequently, the matrix shrinks, that is, the links become more and more concentrated in the upper left corner of the matrix.

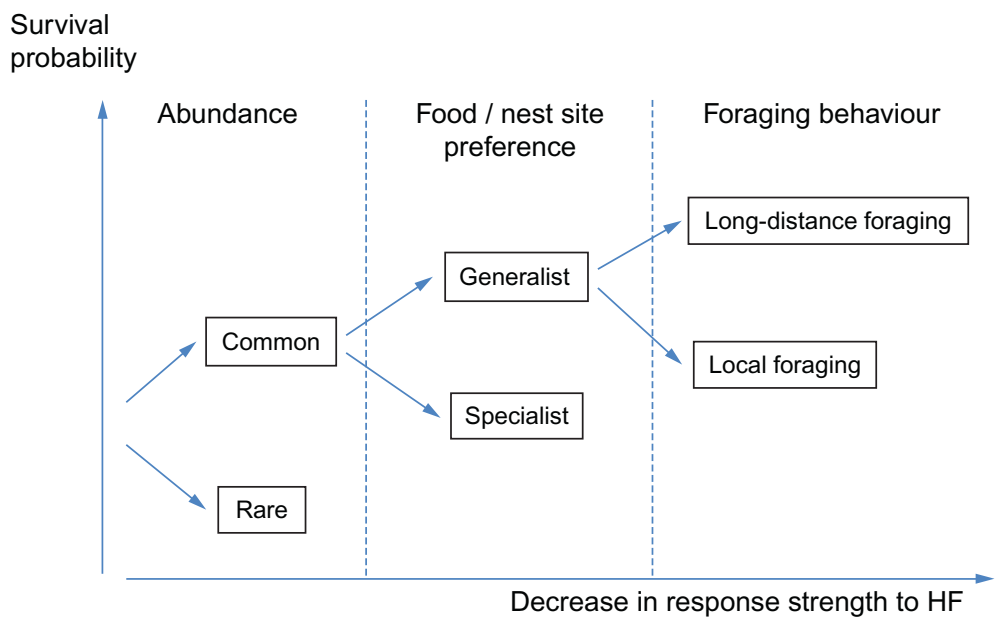

Figure 9 A simplified framework illustrating how the survival probability of pollinator species in response to fragmentation is hierarchically constrained by species traits. 
Local pollination networks trapped in single fragments tend to have higher connectance, because species number decreases and generalists are expected to survive better than specialists (Barbaro and van Halder, 2009; Girao et al., 2007; Koh, 2007; Steffan-Dewenter and Tscharntke, 2002; Williams, 2005; Williams et al., 2009; but see Ashworth et al., 2004). Furthermore, generalists may opportunistically switch or rewire their links depending on resource availability, making them less prone to secondary extinctions (Kaiser-Bunbury et al., 2010) by forcing new links closer to the upper left corner or the interaction matrix (Figs. 8 and 9). This will tend to make the pollinator community more homogenous (Ewers and Didham, 2006). Introduced species, which also tend to be generalists, tend to replace specialists, and this can influence the reproductive output of the plant community (e.g. Didham et al., 1996; Do Carmo et al., 2004) or more so (Dick, 2001).

\subsubsection{Nestedness}

The different ways networks are structured affect the dynamics of their communities and populations: identifying these patterns and their fundamental determinants makes it possible to predict the outcomes of habitat fragmentation. A distinctive property of mutualistic networks and food webs is their nested architecture (Fig. 8; Bascompte et al., 2003; Kondoh et al., 2010). Neutral models can be formulated to track interactions between two species with power law/lognormal (POLO) rank abundance distributions (Halloy and Barratt, 2007), that is, if individuals in two interacting species link randomly irrespectively of any species traits, except abundance ('the neutral theory of biodiversity'; Hubbell, 2001), then the link pattern becomes strongly nested, and even more so than in real networks. Abundance alone may explain $60-70 \%$ of nestedness in empirical networks (Krishna et al., 2008), although perturbations push communities away from a POLO distribution (Halloy and Barratt, 2007). The same neutral model with abundance variation also produces a nested pattern in plant-frugivore networks (Burns, 2006).

Abundance distributions show the importance of short-term disturbance regimes, whereas body-size distributions show more long-term community effects (Halloy and Barratt, 2007). Extending this to networks, certain nested link patterns to reflect systems at or close to equilibrium and deviations from such patterns may therefore be interpreted as a measure of disturbance: although this has yet to be tested formally, it could provide an important new biodiversity metric to gauge higher-level responses to environmental stressors. 


\subsubsection{Link switching}

The strong effect of abundance is often evident, even in spite of the highly dynamical nature of linkage (Olesen et al., 2008, 2010b; Petanidou et al., 2008). An adaptive strategy to cope with spatiotemporal environmental dynamics is link switching or rewiring (Zhang et al., 2011). During network assembly or spatiotemporal changes in environmental conditions, linkage can become increasingly nested as species continuously switch or rewire partners to enhance their fitness gain from other species. Most often, these switches are to species with a higher abundance of more easily exploitable resources, that is, switches towards increasing abundance and trait matching ('The resource attraction principle'; Halloy, 1998). Thus, link switching can place a high selective premium on the ability of an individual to track resources by optimal diet choice and to exploit all resources above a given threshold quality (MacArthur and Pianka, 1966), that is, a more valuable resource becomes a more generalist node in the network, whereas a consumer with a lower choice threshold becomes a more generalist node (Kondoh et al., 2010).

When the landscape fragments, an increase in the intensity of fluctuations of species abundance is expected, and consequently, the ability to do link switching and resource tracking becomes increasingly critical. In mutualistic network models, including link switching into linkage assembly models increases the robustness of networks (Zhang et al., 2011). Consequently, species such as resource specialists that cannot track increasingly unpredictable resources are vulnerable to extinction. In networks, we have two kinds of specialists, ecological and evolutionary: the former because they are rare (or they feed on very few resources) and the latter because of their evolutionary history (low ability to switch resource). Thus, the loss of specialists disappear from networks during fragmentation may arise for different reasons.

\subsubsection{Modularity}

A commonly investigated linkage pattern in pollination networks is modularity (Olesen et al., 2007). The number of modules depends primarily on the size of the network. Modules may further have their own 'deeper' link pattern, for example, submodularity and subnestedness (Fig. 10). Modules are interconnected by species playing specific roles, viz., super-generalists or network hubs and connectors. Three per cent of species in plant-pollinator networks are super-generalists, linking to many species within and outside their own module; $11 \%$ are connectors with a few links, but a high proportion of these links to other modules (Olesen et al., 2007). In the 
early stages of fragmentation modules shrink in size, that is, the nestedness tails are 'cut off', and ultimately only the connectors and hubs are left leaving a topologically simplified network (Carvalheiro et al., 2011). This may initiate an irreversible transition phase or regime shift in network structure and dynamics (Kaiser-Bunbury et al., 2011), because at a certain size threshold modules begin to merge or even disappear. Through extinction and resource switching among generalists, the network slowly collapses by losing its modular structure.

\subsubsection{Body size}

Besides abundance per se, its close correlate body size also has a strong explanatory power of network properties. Body size is an important proxy for many ecological attributes in food webs (Woodward et al., 2005) and maybe also with respect to the response of pollinators to fragmentation. Body size has a huge span in pollinator communities, from tiny $1 \mathrm{mg}$ parasitoids to the largest extant pollinator, the 3-4 kg Malagasy Black-White Ruffed Lemur (Varecia variegata), that is, a difference of six orders of magnitude. For comparison, in a lake food web, there may be difference of 10 orders of magnitude in body size (Woodward et al., 2005). However, the general relationship between linkage level (and thus network position) and body size in pollination network is not clear, although in Caribbean plant-pollinator networks, larger hummingbirds are more specialised than smaller hummingbirds (Dalsgaard et al., 2008).

An equivalent property of the flower is the extent to which floral rewards are accessible. Stang et al. (2006) reintroduced the term nectar-holder depth, that is, the depth from the opening of the flower and down to the surface of the nectar inside the flower. If a flower has a nectar-holder depth $n$, then legitimate pollinators have a tongue length $t \geq n$. If $t<n$, then the link between the species pair is 'forbidden', that is, morphologically constrained (Olesen et al., 2010a). Thus, the relationship between $t$ and $n$ becomes triangular, with generalist pollinators and plants having a high $t$ and low $n$, respectively (Fig. 11; Corbet, 2000; Stang et al., 2006, 2007), as has been observed in several pollinator groups (Borrell, 2005). Tongue length and nectar-holder depth are both correlated with abundance, that is, abundant species have a high $t$ or a low $n$. Since $t$ and body size are positively correlated (Corbet, 2000; Stang et al., 2006) or, in fact, triangularly related (short-tongued pollinators vary considerably in body size, whereas long-tongued species are all large), large pollinators should, in theory, be more generalised. However, the evolution of a long tongue in insects 
A Plants

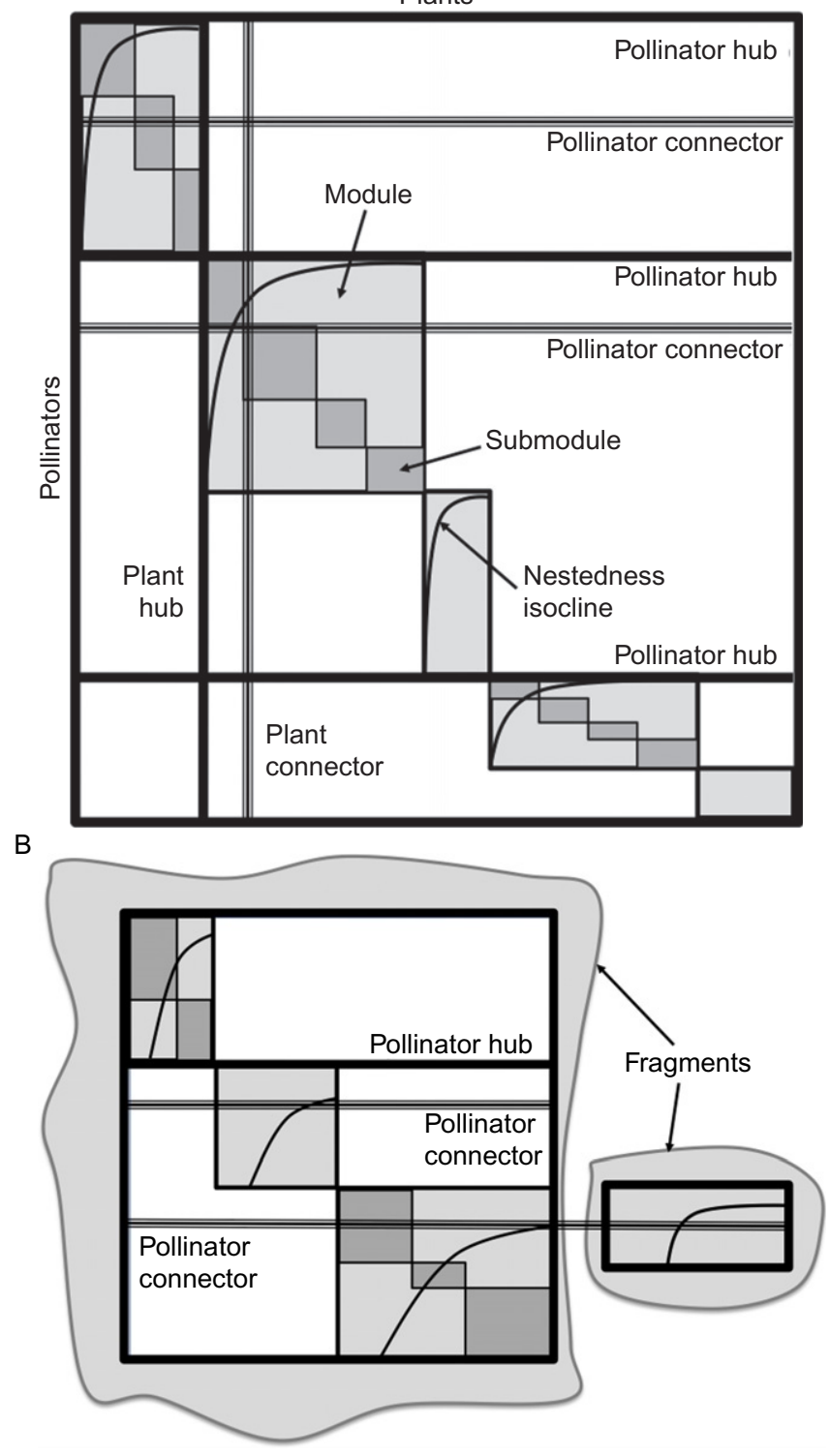

Figure 10 Expected scenario of the destruction of network modularity during habitat fragmentation. (A) An intact network in a non-fragmented landscape. The network has five modules, and three of these are submodular with several submodules (J. M. Olesen, unpublished data); two modules are so small that no modularity can be detected. However, the entire network and four of the modules have a level of nestedness that can be detected; this is indicated with the curved 'isoclines' sensu Atmar and Patterson (1993). 


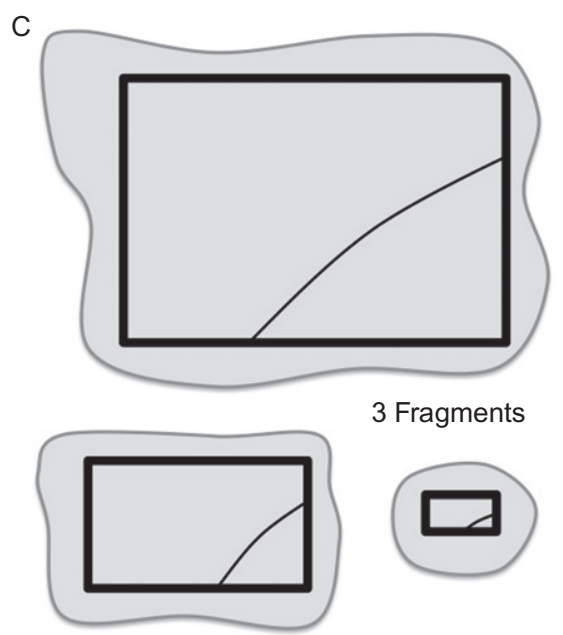

Figure 10-cont'd Most links are to the left of the isocline. Hubs and connectors are shown as bars. (B) The progressing habitat fragmentation has now caused the network to fragment as well. The network is present in two fragments: a large and a small one, and is only connected by one pollinator species. Many of the specialists of both pollinators and plants are gone and only three modules are left in the large fragment. The plant community has mainly lost its outcrossing herbs. The upper left two modules are the same as in (A), whereas the central one is the result of fusion of two modules in (A). This increases connectance as shown by the change in position and shape of the isoclines. A few submodules are still left. (C) The network has now got its modularity completely destroyed by habitat fragmentation. The entire network is now reduced to three single independent modules, each isolated in their own fragment. Most species remaining are generalists, and connectance is high. Many plants from (B) are still alive. They are selfing herbs and long-lived trees, and some of them constitute an extinction debt.

may be a generalist strategy as it allows pollinators to exploit a higher diversity of flowers. Borrell (2005) observed the same triangular relationship between tongue length of euglossine bees and nectar tube length (Fig. 11). In fact, the relationship is, upon closer inspection, more trapezoid-like, indicating that long-tongued bees may have problems with nectar extraction from shallow flowers and that super-generalists have an intermediate tongue length (Fig. 12). These details are, however, still poorly explored.

Stang et al. (2007) simulated extinction scenarios based on field data and found that if abundance is the only determinant then there is no difference in extinction risk between generalists and specialists, whereas an inclusion of 


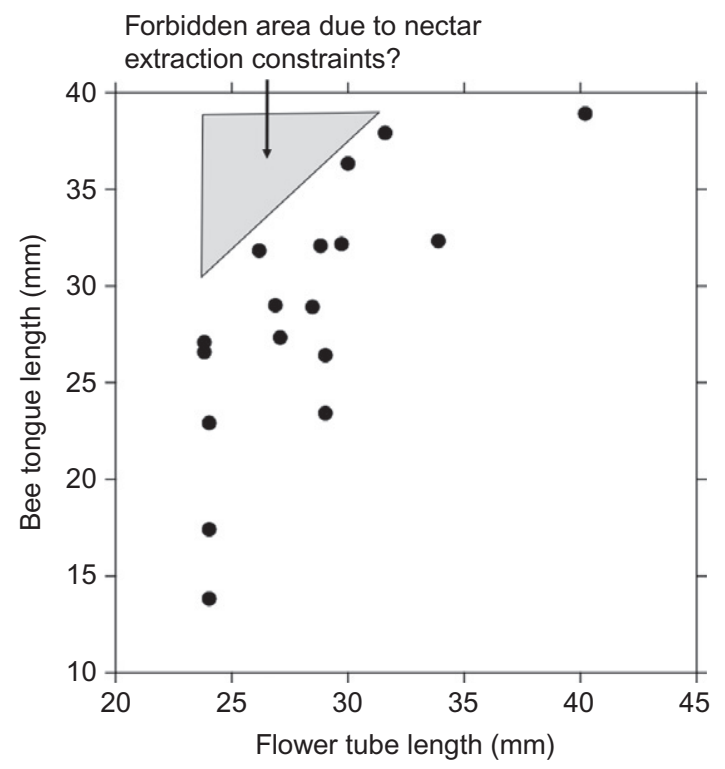

Figure 11 A triangular relationship between flower tube length ( $n$, nectar-holder depth, sensu Stang et al. (2006)) and euglossine bee tongue length ( $t$ ) (data from Borrell, 2005). Upon a closer look, the relationship may be trapezoid, because bees with the longest tongue have problems handling shallow flowers with easily accessible nectar.

nectar-holder depth and tongue length constraints gave an increased extinction risk with increasing $n$ and decreasing $t$.

Body size is also related to mobility and reproduction (e.g. Greenleaf et al., 2007; Nieminen, 1996; Öckinger et al., 2010; van Nieuwstadt and Ruano Iraheta, 1996; Woodward et al., 2005). Expectations are that (i) highly mobile species are less affected by fragmentation than less mobile species; (ii) specialists require larger fragments to fulfil their demands and are also less likely to use the surrounding matrix than generalists and (iii) $r$-species are expected to suffer less from fragmentation than $K$-species, because of their higher reproductive output, which means relatively more emigrants to other fragments. All three hypotheses were confirmed in a study by Öckinger et al. (2010).

The mobility of pollinators affects their population dynamics, genetic structure and life history but also the other species with which they interact (Greenleaf et al., 2007), for example, large-bodied pollinators mediate a longer pollen flow, but also require more energy from their flowers. In many taxa, mobility increases non-linearly with body size (e.g. Steffan-Dewenter and Tscharntke, 1999). The specific movement pattern is of importance 


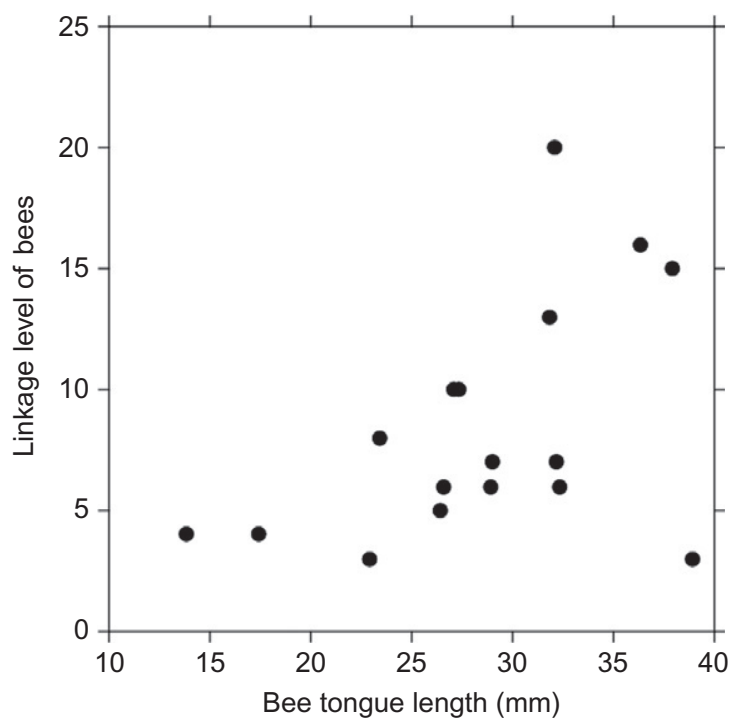

Figure 12 Linkage level, that is, number of visited plant species, of euglossine bees is an increasing function of their tongue length (data from Borrell, 2005). However, linkage level seems to peak between 30 and $35 \mathrm{~mm}$, and the bees with longest tongue avoid some flowers (Fig. 11).

here and the influence of a certain landscape configuration on pollinator movement behaviour can also be incorporated into the analysis (Lander et al., 2011). Some species mediate a more linear pollen flow, for example, large bees and trap-lining hummingbirds, than others and such species may be key hubs or connectors.

In pollination networks, plants with limited modes of attracting pollinators over long distances suffer most from isolation. For example, visual cues tend to be more spatially restricted than scent, which can attract pollinators over considerable distances, for example, hawkmoths (Dudareva and Pichersky, 2006). Amongst generalist pollinators, those that can forage over longer distances due to morphological and behavioural traits can access distant, more isolated resources, and this increases their chance of persistence in fragmented pollination networks. It is important to highlight that this relationship occurs under increasing isolation scenarios, while habitat loss per se is likely to have the strongest adverse effects on large-bodied, long-distance flying animals with high resource requirements.

Plants differ in their dependence on pollinators and seed set by obligate selfers, for instance, should be unaffected by habitat fragmentation, whereas 
facultative selfers may be more affected in terms of seed quality than quantity. Wind-pollinated plants are also expected to suffer less from fragmentation than those pollinated by animals due to their long-distance pollen flow. An important determinant of linkage level in the networkparticipating plant community is flower morphology, especially level of flower openness (accessibility to the interior of the flower), which should increase with the number of pollinator species, although in reality the relationship is more complex (Olesen et al., 2007).

\subsubsection{Four fragmentation scenarios}

Assuming that the response of pollinators to habitat loss and fragment isolation is driven by body size, which could be true for some pollinators such as birds or specific bees, we can outline a simplified framework of how plant-pollinator networks will change in response to fragmentation (Fig. 13). In a system with large and poorly isolated fragments, a plant-pollinator network will consist of many links, including small-, medium- and large-bodied pollinators (Fig. 13A). If fragments become smaller in size, but are similarly isolated, resource availability and nesting sites will decline to critical levels, forcing species to move between fragments to maintain population sizes. Very small species with low resource requirements are more likely to survive, but species and link diversity of intermediate species with low mobility should decline due to a lack of resources within single fragments. Large species, however, should decline due to limited resource availability across fragments in the landscape (Fig. 13B). Maintaining large fragments but increasing the level of isolation will have a weak impact on small species as they can persist within fragments. Large species are likely to survive as they can move between distant fragments due to their large foraging ranges or dispersal abilities (Fig. 13C). The most affected species are expected to be those of intermediate size, with habitat requirements exceeding the fragment size but are unlikely to move the large distances between fragments. The worst-case scenario is that only small fragments remain that are separated by relatively large distances (Fig. 13D). Then, only some small and maybe intermediate generalist species will be able to persist and movements among fragments will be rare. As a consequence, the network is strongly depleted and highly skewed towards small species (Fig. 13D). Given this simplified framework, the number of links in a plant-pollinator network is expected to change in predictable ways as a consequence of habitat loss and isolation (see the two graphs to the right in Fig. 13). 
A

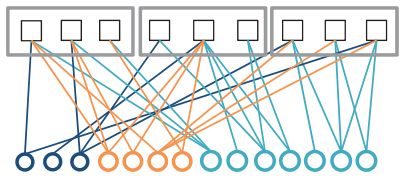

B

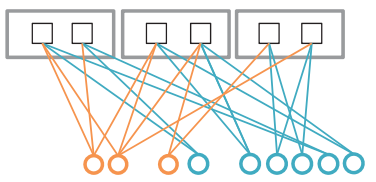

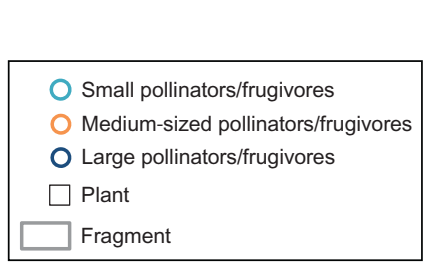

C

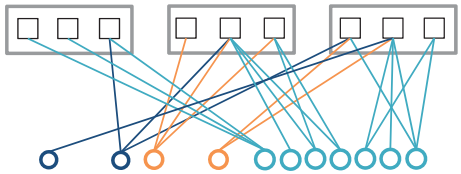

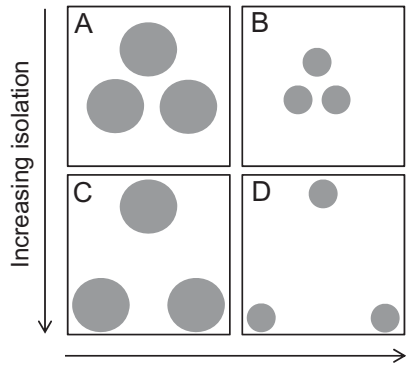

Increasing habitat loss

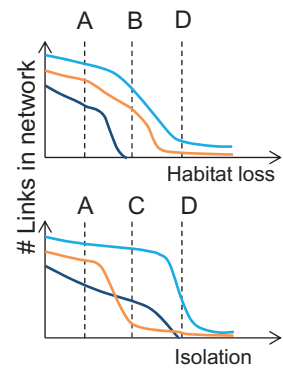

$\mathrm{D}$

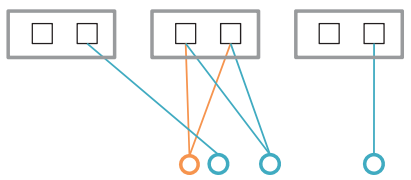

Figure 13 Simplified framework for the response of pollination and seed dispersal networks to habitat loss and isolation. The illustrated framework assumes that body size is the key trait for the response of pollinators and frugivores to fragmentation. In (A), a system with large and poorly isolated fragments contains a plant-pollinator/frugivore network with many links, including small-, medium-sized and large-bodied animals. In (B), fragments become smaller in size (but with a similar degree of isolation), resulting in a decline of small and intermediate species with low mobility and a loss of large-bodied species. In (C), large fragments have an increased level of isolation with weak impacts on small species and more pronounced effects on intermediate and large-bodied species. In (D), a landscape with small and isolated habitat fragments only sustains some small, and maybe intermediate, generalist pollinators or frugivores. The bipartite networks depict hypothetical pollination or seed dispersal networks covering the entire landscape. The number of plant species is kept constant. The two graphs on the right hand illustrate how the number of links in these plant-animal networks changes as a consequence of habitat loss and isolation under this simplified framework.

\subsection{Mutualistic plant-frugivore networks}

Frugivores include a large diversity of taxa, from annelids to elephants, and fish and herps, spanning body masses from a few grams to several tonnes. Plants that produce fleshy fruits and rely on animals for seed dispersal are also 
diverse and differ in fruit size, seedling vigour, phenophase length and so on. In terms of the potential effects of habitat fragmentation, it matters which critical frugivore or plant life strategies are correlated within a network. For example, rare species might be more prone to local extinction following fragmentation (Davies et al., 2004) but they could be occupying peripheral positions in the network, or may be central species. Body mass influences population viability in fragmented landscapes (see e.g. Galetti et al., 2009 for mammals), but we are not aware of any studies to date that have mapped this onto plant-frugivore networks.

The overall response of such networks to fragmentation will depend on the array of species traits in the interacting assemblage. Differential responses and susceptibility among frugivore species will cause variation in incidence functions (Gilpin and Diamond, 1981) of each species across fragments in a complex landscape (Fig. 14), determining variation in survival probability in

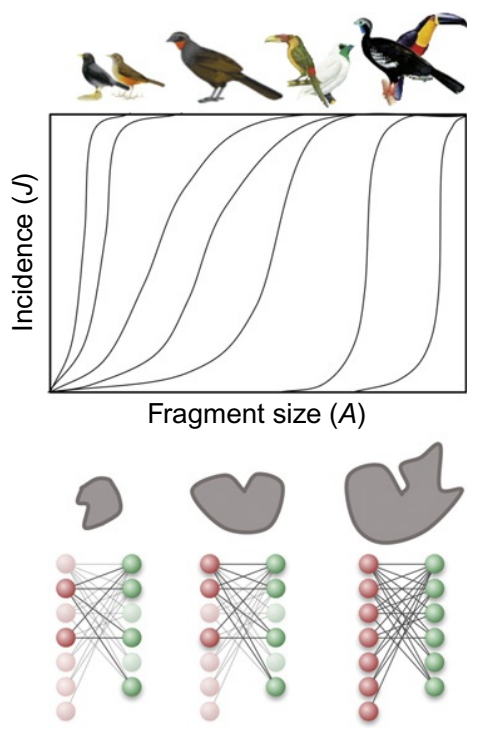

Figure 14 Incidence functions of frugivore species along a gradient of habitat loss (fragmentation). Incidence functions (top) represent the fraction of habitat patches of a given size where a frugivore species is present. Large-bodied frugivores will most likely disappear from small- and medium-sized fragments, while small-bodied frugivores would be the only species present in the small remnants. Variable incidence functions will thus result in differences in specific composition (species richness, relative abundance) of different fragments which, in turn, will cause large variations in network topology and structure (bottom). 
fragments of variable area. This will typically result in different richness and composition of the local plant-frugivore assemblages among fragments, with reciprocal influences between them (Kissling et al., 2007). Patterns of fragment occupation will be driven by colonisation/extinction dynamics, which will depend on how species respond to loss of habitat area and/or increasing distance and isolation among fragmented patches (Luck and Daily, 2003). While Fig. 14 illustrates the depauperation of frugivore assemblages, a similar scenario could be envisaged for fruiting plants, showing, for example, variable incidence functions associated with seed mass or fruit-size variation. The figure is inspired by trends in the composition of avian frugivore assemblages in the Atlantic forest of SE Brazil (Fadini et al., 2009; M. Galetti, personal communication; also see Estarada et al., 1993; Githiru et al., 2002; Graham, 2002). This highly fragmented landscape is impacted not only by habitat loss processes but also by different levels of hunting and poaching that, taken together, drive dramatic local changes in frugivore abundance across fragments (see e.g. Almeida-Neto et al., 2008; Galetti et al., 2009). Large tracts of Atlantic rainforest harbour reasonably complete frugivore assemblages and associated dispersal services to the plants (Fig. 14), yet the smaller fragments contain impoverished local communities that invariably lack the larger frugivores, such as toucans, large cracids and cotingids, whereas the dominant frugivores are thrushes and thraupids. The overall effect is highly transformed interaction networks in the fragments (Fig. 14, bottom) with reductions in degree, and potentially drastic increases in modularity due to loss of large supergeneralist frugivores. This also reduces nestedness, largely due to the missing 'glueing' interactions that the generalists provide (Olesen et al., 2010a).

Plant-frugivore networks could exhibit similar responses to fragmentation to those described for pollination networks (Fig. 13), as the main relevant traits (e.g. body mass) are similar. The plant-frugivore networks in landscapes with large and well-connected fragments will harbour reasonably complete networks, with diverse interactions in nested assemblages (Fig. 13A). Most frugivorous birds, for instance, include generalised foragers with flocking behaviour and seasonally altitudinal migrants; many should have high mobility and dispersal abilities. Santos et al. (1999) reported that drastic alterations of local thrush assemblages in juniper fragments in central Spain mainly occur in the smallest fragments (also see Luck and Daily, 2003). If fragment area becomes reduced, but still maintaining good connectivity, some large species may still be lost because of reduced home range sizes and 
resource abundance. As for plant-pollinator networks, small species with reduced resource requirements are likely to survive (e.g. small avian frugivores with mixed diets, not relying extensively on fruit), while species and link diversity of small- and medium-bodied species with low mobility and large species should decline (Fig. 13B). With increased isolation, small species may persist in medium-sized and even small fragments, whereas the persistence of larger species will depend on their ability to disperse among fragments (Fig. 13C). As with the pollination networks, seed dispersal interaction networks in landscapes with both reduced fragment area and poor connectivity should be more prone to collapse (Fig. 13D; see e.g. Santos et al., 1999). Then, only some small, and maybe intermediate, generalist species will be able to persist and movement among fragments will be rare. The small fragments cannot support large species, and the network is again strongly biased towards a few small species (Fig. 13D).

Fragmentation and habitat loss will ultimately induce the loss of specific nodes (either plants or animals), reduced population densities of mutualistic partners, resulting in dramatic losses of important functional attributes. For example, in some Pacific islands, populations of flying foxes are periodically reduced by hurricanes to a point beyond which their capacity to disperse the seeds of big-seeded trees decreases dramatically (McConkey and Drake, 2006). Such functional losses will not take place at random, but will be concentrated in certain species, like larger frugivores and large-fruited plants. In summary, the main consequences of fragmentation for plant-frugivore networks will depend upon the extent that key traits determining susceptibility of species correlate (or match) with traits that define their functional roles in the network.

\subsection{Mutualistic plant-ant networks}

Symbiotic and free-living plant-ant mutualisms are organised in networks that differ markedly in their structure (Blüthgen et al., 2007; Fonseca and Ganade, 1996; Guimarães et al., 2007): for example, those that include extrafloral nectaries are often nested, whereas symbiotic, plant-ant networks are always strongly modular (Guimarães et al., 2007). These correlations between biological attributes and network structure can be used to infer likely responses to habitat fragmentation.

If habitat fragmentation affects ant species of distinct body sizes differently (see Section 5.3), the same will be true for the highly and poorly connected species. At present, the underlying mechanisms linking ant body size to the number of interactions and the degree of overlap among partners are unknown, making it difficult to predict the consequences for species 
networks, even if there is a clear body size-biased effect of habitat fragmentation on species composition (however, see Chamberlain and Holland, 2009). Thus, it is fundamental to develop a better understanding of how ant body size is related to network structure in plant-ant interactions to predict the fate of these networks facing habitat fragmentation.

Phylogeny is an important predictor of the structure of symbiotic networks (Fonseca and Ganade, 1996), which are composed of modules that often contain closely related ant and/or plant species. This strong association between phylogeny and network structure is predicted as a consequence of a 'complex coevolutionary handshaking' among interacting partners (Thompson, 2005). This relationship should enable responses of plant-ant networks to fragmentation to be predicted, if sensitive groups of taxa can be identified a priori: if the phylogenetic signal is very strong, such as in symbiotic plant-ant interactions, susceptibility traits and traits shaping the role of a species within a network are likely to be strongly correlated.

Key questions that need to be addressed include how nestedness will alter with changes in ant species richness and composition: the current evidence, although still limited, suggests the nested structure of extrafloral nectary networks to be robust to species turnover and invasions (Diaz-Castelazo et al., 2010). It is also important to understand how the strong modularity of symbiotic networks is affected by habitat fragmentation, which has the potential to cause the emergence, loss or even fusion of modules (e.g. via invasions of generalist ant species). In a fragmented landscape, one could imagine the creation of a mosaic of plant-ant networks varying in species composition and consequently in nestedness and modularity.

\subsection{Antagonistic food webs}

The effects of fragmentation on food webs have been surprisingly overlooked. In terrestrial systems, we can envisage fragmented networks in the classical biogeography sense when they are situated within islands within an aquatic matrix. An example of this comes from recent work carried out in Ireland (McLaughlin et al., 2010). The Gearagh woodland, located in the floodplain of the River Lee in County Cork, is composed of a complicated braided river system composed of approximately 13 channels, each 1-7 m wide. The main channels are stabilised by tree roots, which create a mosaic of small islands due to the accumulation of detrital material and fallen trees over time. A food web study, examining the trophic structure of the invertebrate community on series of 16 islands, ranging in size from 4.5 to 
$40.8 \mathrm{~m}^{2}$ found that, on average, the larger islands contained more species and links than the smaller islands, and network structure consequently differed markedly among fragments (Fig. 15).

Fragmentation of food webs can also occur in other lateral (i.e. across landscape) and temporal dimensions, as well as via fractal branching pattern
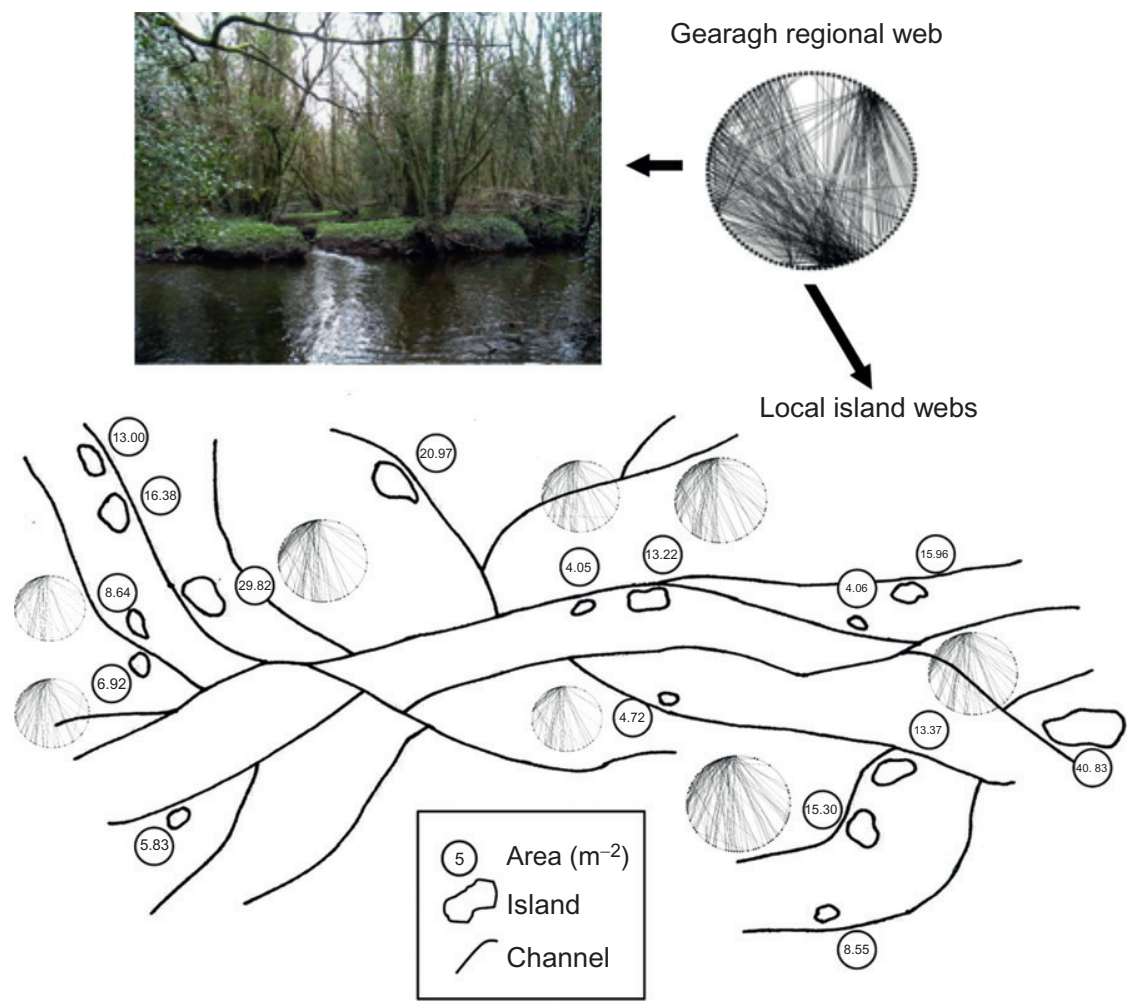

Figure 15 Schematic representation of the riverine network with the Gearagh forest, Ireland. Individual islands are inserted beside the river channel in which they were located (McLaughlin et al., 2010). The Gearagh is a complicated braided river system composed of approximately 13 channels, each 1-7 m wide. The study site was comprised of a small proportion of these channels. The stabilising effect of the tree roots within the main river channels, in conjunction with the accumulation of detrital material and tree falls, has resulted in the above mosaic of small islands. The diameter of the web from each island is scaled linearly with species richness: the larger webs are found in the larger fragments. Note: each web contains the same number and positioning of nodes as in the global web: solid black nodes represent macroinvertebrate taxa present within the depicted web and grey nodes indicate taxa present in the global web but absent from the depicted web. 
dimensions (e.g. in river networks) (Box 1; Fig. 16). Additionally, vertical fragmentation, which is even more rarely considered, can occur, such as in mountainous regions (Box 8; Fig. 17).

The loss of large consumers at higher trophic levels due to habitat fragmentation should result in a decreased overall trophic height of the food web, driven by shorter food chains (e.g. Byrnes et al., 2011; O'Gorman and Emmerson, 2009; Woodward et al., 2012). This could also lead to an increase in the proportion of top consumers relative to intermediate species, as the latter are effectively promoted to the termini of food

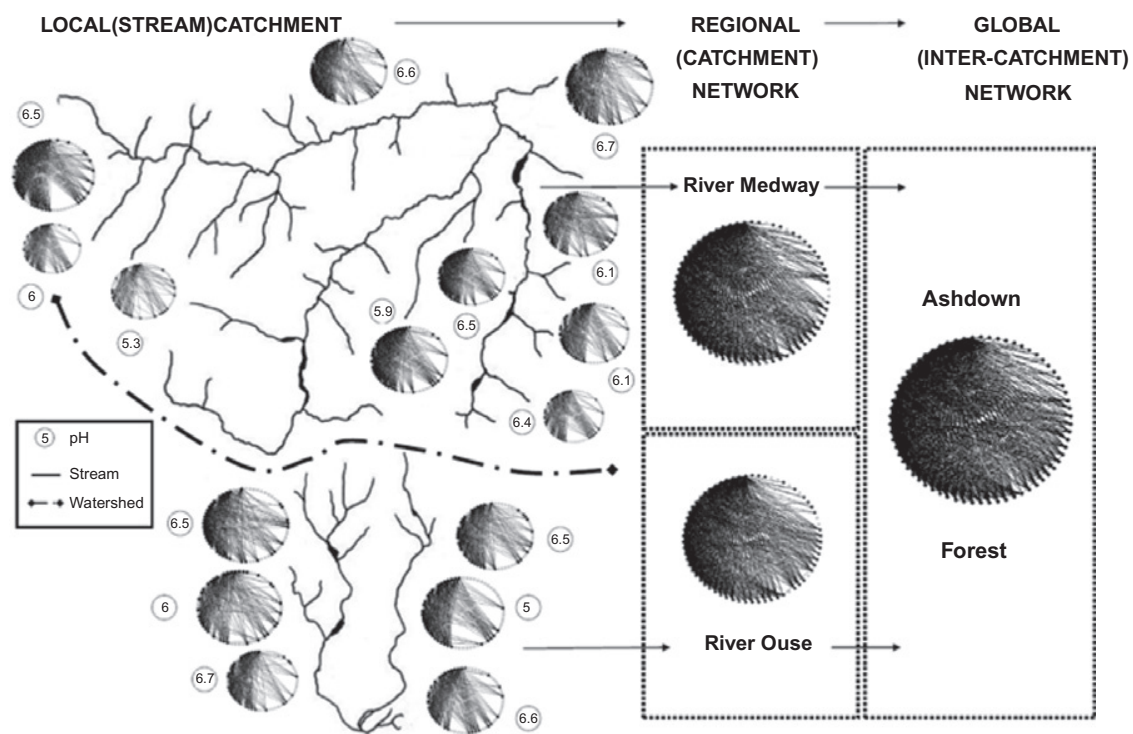

Figure 16 Ecological network structure of stream food webs from the Ashdown Forest, UK, shown from local to regional to global networks. Note: each web contains the same number and positioning of nodes as in the global web: solid black nodes represent macroinvertebrate taxa present within the depicted web and grey nodes indicate taxa present in the global web but absent from the depicted web (Fig. 15). Web diameter has been scaled to the number of nodes as a \% of those in the global web: thus the smallest web also contains the fewest species. All streams are headwaters of either (a) River Medway or (b) River Ouse, which are separated into discrete watersheds (separated by the dashed east-west line) that flow predominantly either north or south into the sea. Individual networks are constrained by the 'hard' boundary of the water's edge and the 'soft' boundary of a physiochemical gradient (indicated by mean stream $\mathrm{pH}$, within circles adjacent to each web). All individual streams can be viewed as a fragmented component of the catchment network, which in turn is a component of the global network. The increasing complexity of the network can be seen as the number of nodes and consequently the number of interactions increases once the fragmented nature of the landscape and habitat is discounted. 


\section{BOX 8 Fragmentation of Mountainous Aquatic Food Webs}

Habitat fragmentation is typically considered in lateral (i.e. across landscape) and temporal dimensions, but vertical fragmentation is also possible, for example, in mountain ecosystems. At high altitudes, glacier retreat and changes to the magnitude of snowpack accumulation and their duration are likely to cause major changes to aquatic ecological networks within an already fragmented landscape (e.g. Brown et al., 2007, 2012; Finn et al., 2010; Jacobsen et al., 2012; Milner et al., 2009). There are strong upstream to downstream gradients in aquatic biological assemblages in these systems, driven predominantly by changes in stream water temperature and the geomorphological stability of the river channel (Milner et al., 2001). Consequently, alpine river food webs are highly fragmented along even short distances (kilometres), with high turnover of species, food web links and species' contributions to secondary production (e.g. Fig. 17). In non-glacial mountain rivers, altitudinal pressure effects on the saturation of dissolved oxygen can impart major effects on community composition (Jacobsen, 2008). Montane aquatic ecosystems that rely on meltwater are particularly susceptible to fragmentation, particularly in situations where decreases in meltwater production lead to drying of some river sections (e.g. Malard et al., 2006). Natural occurrences of river ecological network fragmentation are also evident where lakes introduce discontinuities into the system (Milner et al., 2011; Monaghan et al., 2005). Alpine lakes lead to notable changes in community composition and the relative abundance of morphological and biological traits relative to the nearby flowing waters, but may be insufficient to prevent insect dispersal and thus genetic differentiation within river valleys (Monaghan et al., 2002). Fish may be restricted to lower altitudes due to thermal or geomorphological barriers (e.g. falls, canyons; Evans and Johnston, 1980), thus preventing their upstream migration to avoid warming. Therefore, the more productive and species-rich aquatic food webs at lower altitude sites (e.g. Fig. 17) may fragment as some mobile organisms such as invertebrates are able to migrate to higher altitudes. The immigration of 'lowland' species to higher altitudes may also upset the balance of these food webs, causing fragmentation but also succession. Additionally, at higher latitudes, there may be fragmentation as the range of some amphibians (e.g. Pyrenean Brook Newt, Calotriton asper) expands from currently clear water habitat (Parc National des Pyrénées, 2005) into glacier-fed rivers that are receiving less meltwater (and proportionally more groundwater) with glacier retreat.

chains as the largest higher-level predators are lost (see O'Gorman and Emmerson, 2010; Woodward et al., 2012). Loss of large species at high trophic levels is also likely to result in reduced linkage density (Montoya et al., 2005; O'Gorman et al., 2010) and connectance (O'Gorman and Emmerson, 2010) within local networks, as well as reduced 


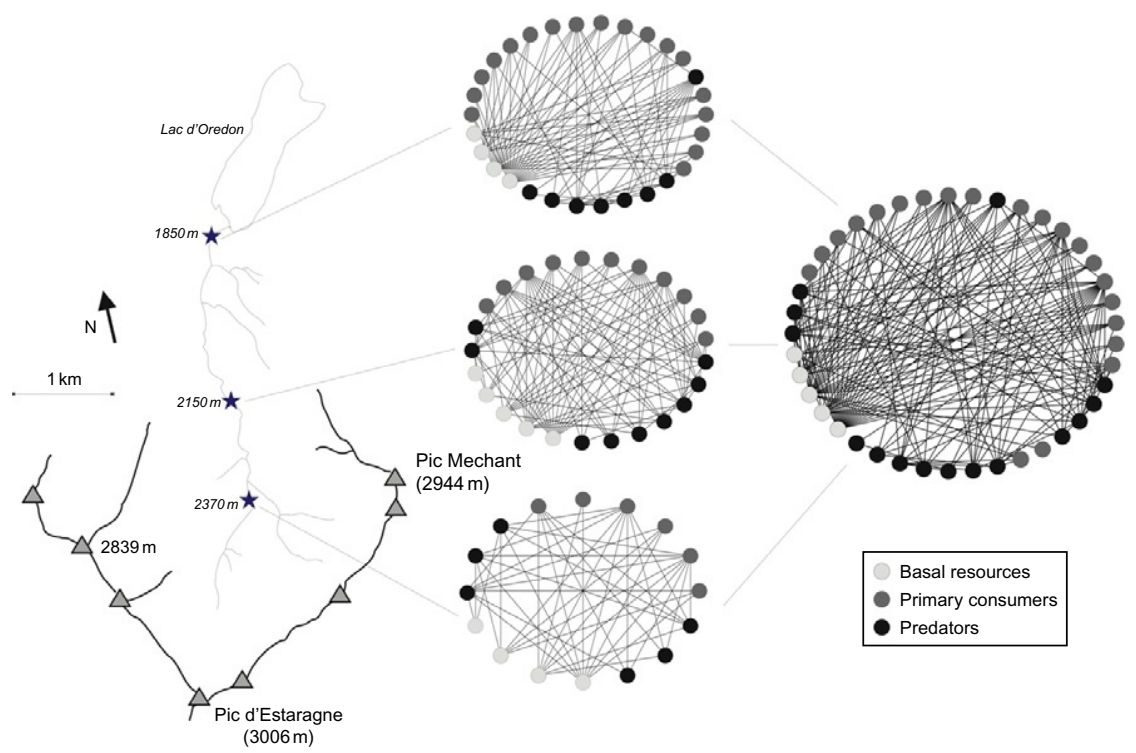

Figure 17 Stream benthic food webs along an altitudinal gradient in the Estaragne catchment, French Pyrénées. Light grey circles denote basal resources; dark grey denotes primary consumers; black denotes predators. Three food webs are displayed for (i) $2370 \mathrm{~m}$ altitude, maximum water temperature $\left(T_{\text {max }}\right)=4.5^{\circ} \mathrm{C}$, no. species $(S)=$ 16 , no. links $(L)=46$, secondary production $(2 P)=4.9 \mathrm{~g} \mathrm{~m}^{-2}$ year $^{-1}$; (ii) $2150 \mathrm{~m}$ altitude, $T_{\max }=8.5^{\circ} \mathrm{C}, S=25, L=93,2 P=6.55 \mathrm{~g} \mathrm{~m}^{-2}$ year $^{-1}$ and (iii) $1850 \mathrm{~m}$ altitude, $T_{\max }=138{ }^{\circ} \mathrm{C}, S=30, L=87,2 P=7.6 \mathrm{~g} \mathrm{~m}^{-2}$ year $^{-1}$. The individual food webs are fragmented as the individual study sites are separated by soft boundaries. Together, these food webs combine to a composite web of 41 species with 164 links. Figures redrawn from Lavandier and Décamps (1983) and Lavandier and Céréghino (1995).

compartmentalisation, which could make the web less robust to secondary extinctions (Dunne et al., 2002), although this is not necessarily the case if there is high redundancy in the system (Woodward et al., 2012). Large species may have weak per unit biomass interactions with their prey and high functional uniqueness (O'Gorman et al., 2011), so their extinction could increase the overall interaction strength within the system. This may reduce stability (see McCann et al., 1998; Neutel et al., 2002), while loss of functional trait diversity will alter ecosystem process rates and functioning (Petchey and Gaston, 2006).

Body-mass-driven extinctions due to habitat fragmentation may cause an overall increase in the predator-prey body mass ratio, assuming that larger predators eat prey closer to their own body mass (Brose et al., 2006). Smaller predator-prey body mass ratios have been linked to longer food chains due to their stabilising properties (Jennings and Warr, 2003; Jonsson and Ebenman, 1998; however see Mulder et al., 2009; Reuman et al., 2009), 
so increases could raise the probability of catastrophic phase shifts or total collapse. Conversely, in systems where large predators are considerably larger than their prey (e.g. fish eating invertebrates vs. invertebrates feeding on other invertebrates), the loss of these consumers could increase stability of the food web, as appears to be the case in headwater streams where fish are lost due to habitat loss and fragmentation arising from chemical and/or physical barriers (Layer et al., 2010, 2011).

The response of freshwater food webs to fragmentation by droughts (Box 1) has been characterised recently by manipulating flows in a series of artificial stream mesocosms (Ledger et al., 2008, 2011, 2012; Woodward et al., 2012; Fig. 18). These model systems reflected the abiotic conditions, biodiversity and food web properties of natural streams (Brown et al., 2011; Harris et al., 2007; Ledger et al., 2009). The results of this fragmentation experiment revealed some dramatic impacts on the food webs: consistent with the higher trophic rank hypothesis (e.g. Holt, 1996), top predators' production declined by $>90 \%$. Among the primary consumers, production of shredder detritivores was also suppressed (by 69\%), whereas the base of the food web was relatively unaffected (Ledger et al., 2011, 2012). Contrasting responses were evident among functional groups, ranging from extirpation to irruptions in the case of small midge larvae, although production of most species was suppressed. The ratio of production to biomass increased, reflecting a shift in production from large, longlived, taxa to smaller taxa with faster life cycles (Ledger et al., 2011). Fragmentation by drought caused high mortality and the partial collapse of the food web from the top-down (Ledger et al., 2012) as well as reversing successional dynamics of benthic algal assemblages (i.e. basal resources), with effective colonists replacing competitive dominants (Ledger et al., 2008, 2012). The general shift in biomass flux from large to small species could not fully compensate for the overall biomass flux. Many other network characteristics (e.g. connectance) were, however, conserved, suggesting some higher-level properties might be conserved even when exposed to extreme perturbations (Woodward et al., 2012).

Fragmentation can also affect marine food webs (Box 1). Coral bleaching creates fragments of surviving coral surrounded by reef pavement and coral rubble, with consequences for top-down control as average food chains shorten, generalist species proliferate and phase shifts may occur (Hughes, 1994). Simulations of fragmentation processes in Caribbean coral reefs indicate that species losses due to body size or diet constraints will lead to decreases in 
Regional web (all spatial replicate webs and both treatments combined)

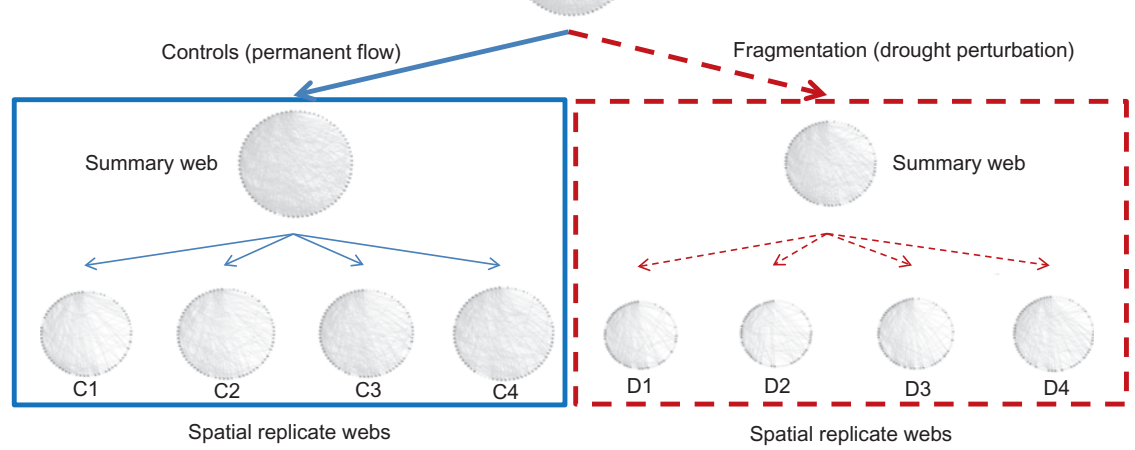

Figure 18 Impacts of habitat fragmentation caused by drought in experimental stream food webs: results from a long-term field experiment in artificial streams (Brown et al., 2011; Ledger et al., 2008, 2009, 2011). Drought can have patchy effects in river networks and individual stream channels can be viewed as fragmented patches in the wider riverscape. Note the two experimental treatments (monthly drought disturbance vs. permanent flow) were randomised spatially among the eight stream channels, but are grouped into two blocks here for illustrative purposes. The diameter of the circular webs is scaled according to species richness relative to the global web for the combined network. Solid nodes represent species present in a given web; open nodes represent those found in the global but not in local web (Figs. 16 and 17). Droughts simplified the networks with marked impacts on large rare species high in the web.

number of links and changes in connectance and food chain length (Fig. 19). Human-induced fragmentation in seagrass food webs could further lead to fewer trophic groups and top predators, lower maximum trophic levels, shorter food chains and prey-dominated communities (Coll et al., 2011). In kelp forests, habitat loss and fragmentation due to storms simplify marine food webs, mainly by decreasing diversity and complexity at higher trophic levels, resulting in shorter food chains (Byrnes et al., 2011). The effects of habitat fragmentation on food webs, although little studied, can be pronounced.

\subsection{Antagonistic host-parasitoid networks}

Besides food webs, several examples from other multitrophic systems give an indication of how antagonistic host-parasitoid networks may be affected by fragmentation (Cronin, 2004; Kruess, 2003; Thies et al., 2005). However, 


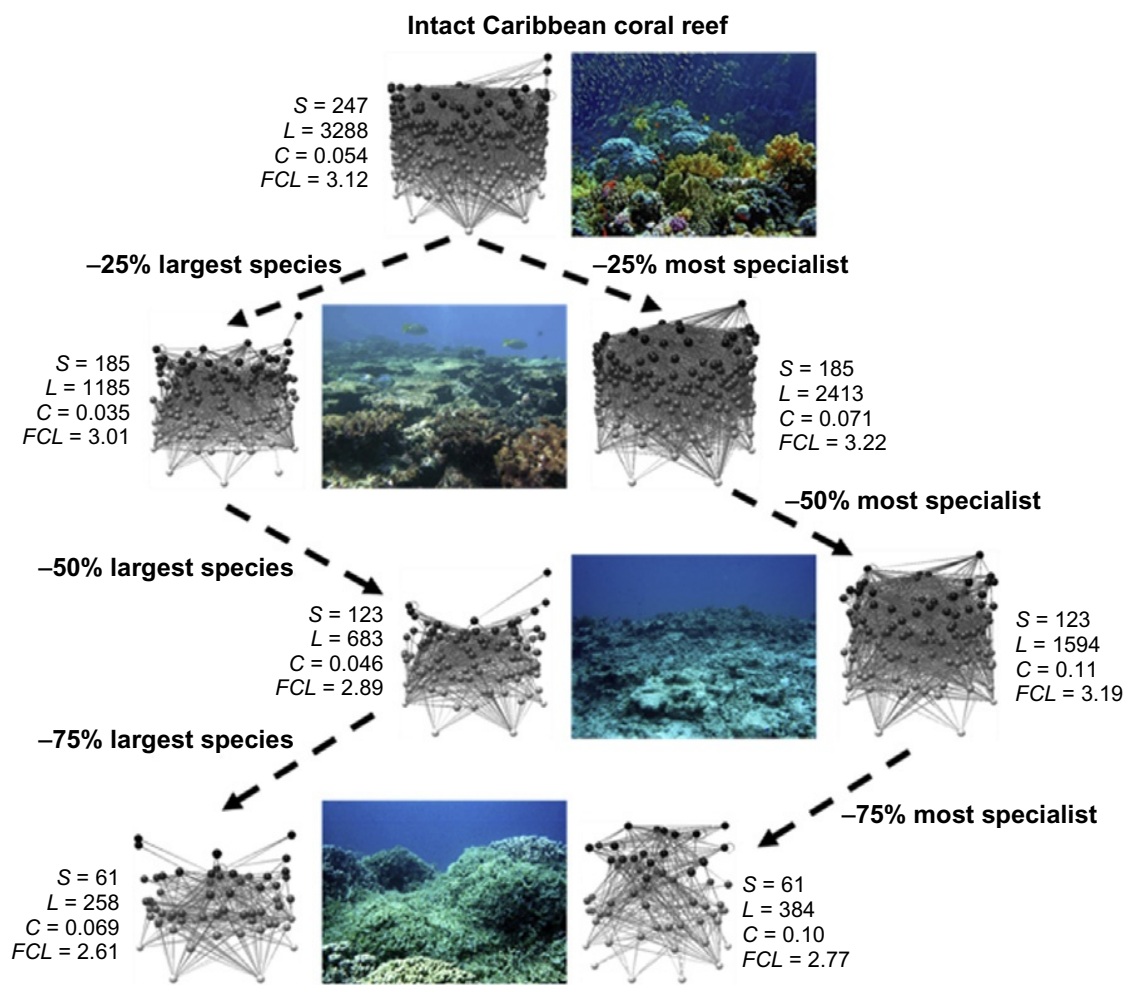

Figure 19 Simulated consequences of fragmentation-driven extinction scenarios on the network properties of a Caribbean coral reef. As species $(S)$ are lost according to body size, the number of links $(L)$ in the web decreases exponentially, leading to unpredictable fluctuations in connectance $(C)$ and a linear decrease in mean food chain length ( $\mathrm{FCL}$ ). As species are lost according to diet specialisation, $L$ decreases linearly, leading to an overall increase in $C$ and $F C L$ until a critical threshold is reached and the system undergoes a phase shift to a new state (e.g. macroalgae dominated). Coral reef photos are used by kind permission of José Eduardo Silva, Stephen Leahy, Nick Graham and James Acker (respective photo credits, from top to bottom).

because species respond differently to fragmentation effects, it is currently not possible to predict whether some will compensate for others, and therefore how overall parasitoid-host network structure will be affected, although progress is being made in this area. For instance, in restored and adjacent intensively managed meadows, the abundance and parasitism rates of bee hosts decreased with increasing distance from restored meadows and the diversity of interactions declined more steeply than the 
diversity of species (Albrecht et al., 2007). This suggests a strong impact of habitat fragmentation on trophic networks and that interaction diversity might decline more rapidly than species diversity in fragmented systems. Another study examined host-parasitoid networks of specialist leafminers and their parasitoids on individual oak (Quercus robur, Fagaceae) trees in different landscape contexts (Kaartinen and Roslin, 2011). Isolated patches had fewer species and different composition than well-connected patches, but the quantitative metrics of network structure (interaction evenness, linkage density, connectance, generality or vulnerability) were unaffected, indicating some degree of functional compensation across species. More case studies are now needed to test the generality of fragmentation effects in host-parasitoid networks.

\subsection{General effects of habitat fragmentation on network properties}

The examples above illustrate that the properties of mutualistic and antagonistic networks can be strongly affected by habitat fragmentation, although this field is still very much in its infancy (Burkle and Alarcón, 2011; Fortuna and Bascompte, 2006; Gonzalez et al., 2011). Simulation studies indicate that mutualistic networks can be buffered to some extent against habitat fragmentation (Fortuna and Bascompte, 2006). Real communities might persist for longer but start to decay sooner than randomly generated in silico communities, with resilience against fragmentation being provided by degree or link heterogeneity (Jordano et al., 2003), nestedness (Bascompte et al., 2003), compensatory responses and/or redundancy (Ledger et al., 2012).

Species and link richness vary with habitat area, with the latter seemingly being more sensitive to fragmentation than the former (Sabatino et al., 2010), that is, as a local habitat shrinks, interactions are lost faster than species. This might be related to a reduced abundance of species (without initially going extinct), which reduces interaction probability (encounter rate). It might also be a consequence of several species having more than just one interaction, although ecological networks are highly skewed (Jordano, 1987). Habitat fragmentation influences the strength and timing of species interactions, which can cause cascading secondary extinctions in networks (Solé and Montoya, 2006; Terborgh et al., 2001; Tylianakis et al., 2008). 
Nestedness and other network structure parameters are often determined by relative species abundances (Krishna et al., 2008). Given that habitat fragmentation reduces abundance (Hadley and Betts, 2012), nestedness should change with increasing fragmentation. Fragment area and trophic level or dietary guild identity are likely to influence the degree of nestedness in fragmented landscapes (Hill et al., 2011). Furthermore, effects of vegetational aggregation (clustering of plants in a landscape) and mobility of species can affect network properties, especially in antagonistic and plant-frugivore networks, while these influences on plant-pollinator network structure may be less pronounced (Morales and Vázquez, 2008).

Habitat fragmentation can also influence network substructure (modularity or compartmentalisation) and the extinction of top consumers may disconnect spatially segregated ecological networks and thus increase modularity. The opposite effect may be triggered by the invasion of hypergeneralist species, which connect distinct modules and reduce modularity in fragmented landscapes (Aizen et al., 2008). At some point, the local network must reach a critical level, below which modularity no longer exists. Thus, the modular structure disintegrates before the local network disappears completely. Using a spatial network approach, modularity analysis may lump similar fragments together based on their constituent species (for a biogeographical example, see Carstensen et al., 2012). Fragments within the same landscape might therefore have more similar dynamics and trajectories of change in species composition than those in other landscapes ('landscape-divergence hypothesis'; Laurance et al., 2007), which could be tested with modularity analyses if data from several fragmented landscapes are available.

Fragment size and isolation affect the composition of ecological networks: while large areas can support most interactions needed for normal functioning, small fragments will contain only a core group of species and fewer important interactions (see Section 7). The degree of specialisation of a species will determine whether it can persist, with generalist mutualists being least likely to suffer extinction (Fortuna and Bascompte, 2006). Matrix quality also determines the impact of fragmentation on networks as it defines landscape permeability. Network susceptibility will thus depend on species composition, interaction types and landscape properties (Bender and Fahrig, 2005): one could argue that large fragments have a higher conservation value due to the increased likelihood of modularity, which reduces the risk of the spread of disturbances. 


\section{HABITAT FRAGMENTATION IN A META-NETWORK CONTEXT}

Meta-population ecologists envision a natural landscape as consisting of suitable habitat patches (fragments) containing local species populations, connected through dispersal (Hanski, 1998). Local extinction and colonisation create a dynamic state (Hanski and Simberloff, 1997), determined by the isolation of the patches (including matrix permeability) and the reproductive potential of each population. Likewise, the extinction probability in a given patch is related to its isolation (how likely the patch is to receive immigrants), area (small patches often have smaller populations, which are more vulnerable to stochasticity) and quality (MacArthur and Wilson, 1963; Hanski, 1998, 1999). Thus, patches are often divided into sources and sinks, depending on whether the populations are producing an excess of individuals or are relying on a net input to persist (Hanski and Simberloff, 1997).

Single-species meta-population models have been extended to models of two or more interacting species, which, through antagonistic or mutualistic interactions, modify the dynamics of each other, alongside traditional metapopulation dynamics (extinction and colonisation) (Hanski, 1999; Nee et al., 1997; Prakash and de Roos, 2004). Intriguingly, Nee and May (1992) demonstrated that species interactions (superior competitor and inferior coloniser vs. inferior competitor and superior coloniser) may change species composition in remnant patches in a fragmenting landscape. The complexity of the mathematical models describing the dynamics of meta-populations increases rapidly as more species are added (Klausmeier, 2001), but in reality, habitat fragmentation affects whole communities of multiple species interacting simultaneously.

With an implicit reference to meta-populations and meta-communities (Hanski, 1999; Hanski and Gilpin, 1997), meta-networks can be defined as a set of spatially distributed local networks connected by species dispersal and influenced by colonisation and extinction dynamics (Fig. 20). These meta-networks can be considered as a combination of spatial and ecological networks (see Section 2) in a meta-population context. To date, little work has been done in this field, although such approaches offer a promising means for assessing (1) dispersal and movement between local networks, (2) the colonisation and extinction of species in local networks and (3) implications of habitat fragmentation on the topology of local networks. 

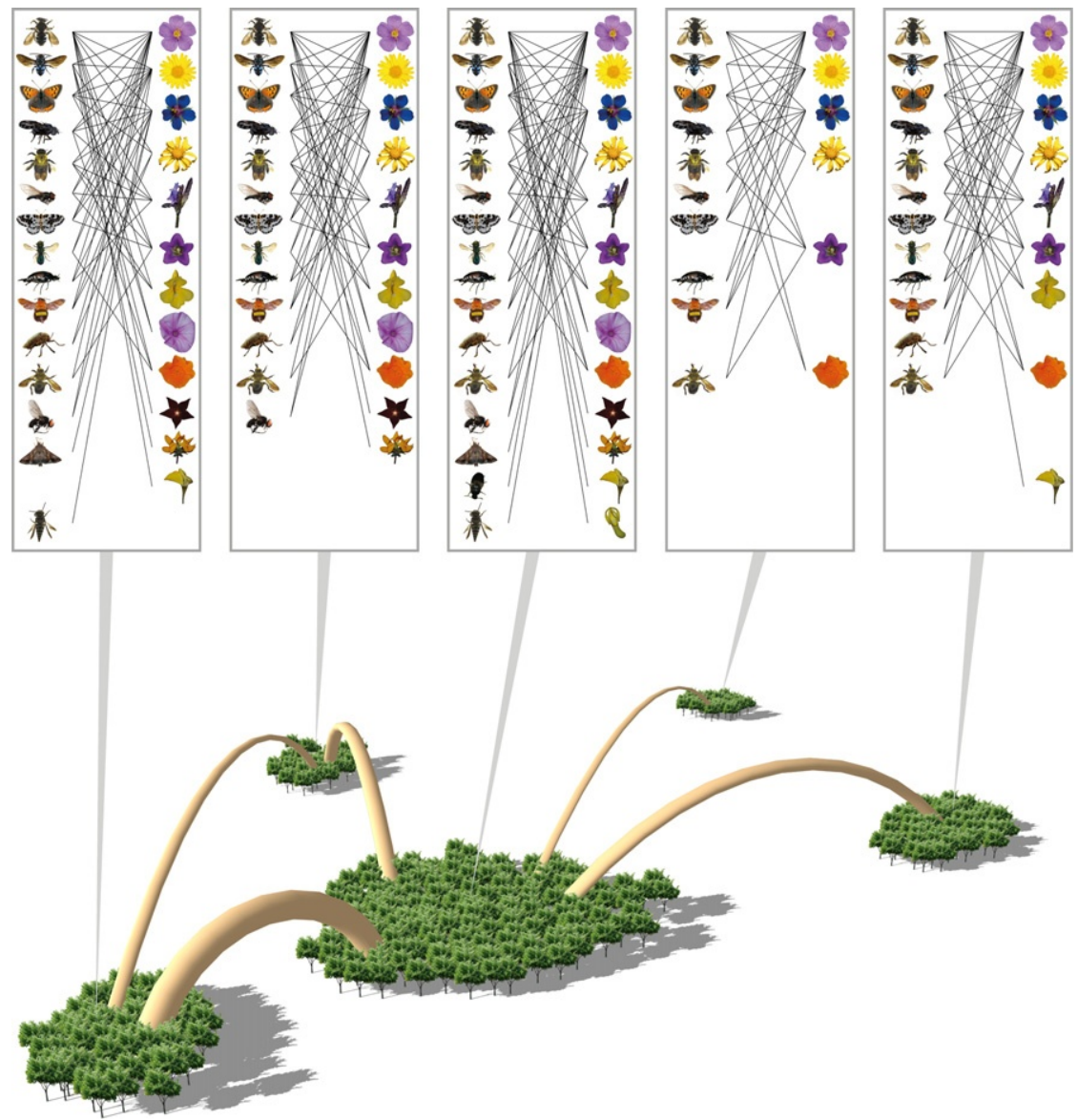

Figure 20 Ecological networks in a meta-network context. A fragmented landscape consists of local habitat fragments separated by a more or less permeable matrix. Within each habitat fragment, networks of interacting species can be found which differ in their structure and degree of complexity. A fragmented landscape usually contains fragments of different sizes at different degrees of isolation. Here, it is illustrated how a big fragment, containing many interacting species, may support minor fragments via species dispersal and thereby contribute to the maintenance of species composition and local network structure. However, small fragments are not able to support all species and isolated fragments are less likely to receive immigrants, and thus, some species and interactions (hence, links) will be lost. The thickness of the 'bridges' between fragments represents the relative degree of species movement between them. In some cases, dispersal might be bidirectional while in others (especially between large and small fragments) movement might be unidirectional, that is, from a source to a sink. Note that the most specialised species are likely to be the most vulnerable. A different effect on network structure will emerge if criteria other than specialisation are used. 


\subsection{Meta-networks and dispersal}

Dispersal and movement of species among patches may be density-dependent or density-independent (Hansson, 1991; Kuussaari et al., 1996; Sæther et al., 1999 and references therein). Low-density dispersal may, for example, be due to a failure in locating mates or specialised mutualists. When locating specialised mutualists, it is the density of the interacting partner that is critical for moving and dispersing. High-density dispersal, on the other hand, may be a result of resource competition among conspecifics or other species. Here again, the network approach offers promise, as it not only specify who is interacting with whom, but also who is interacting with the same partner and thereby, potentially, competing for the same resources. If a landscape becomes more fragmented over evolutionary relevant time scales, increased (mean and long-distance) dispersal rates will be selected for. For example, some sphingid male hawkmoths have evolved a strong olfactory sense enhancing their dispersal success and experienced metanetwork-level selection for increased dispersal rates (Hanski, 1999).

Within a meta-population, dispersal may be unidirectional, that is, from a source to a sink (Pulliam, 1988), and analogies may be drawn with metanetworks (Fig. 20). In meta-population theory, a population is regarded as a source, if the intrinsic rate of increase $(r)$ of the population is $r>0$, and a sink if $r<0$ (Leibold et al., 2004; Pulliam, 1988). However, a local network could be a source for some species but a sink for others (Pulliam, 1988). Thus, when assigning the label source or sink to a local network, a better approach might be to look at the overall intrinsic rate of increase for all the species. As such, a local network could be regarded as a source, if it has a net increase in species $(R>0$, where $R$ equals the number of species with $r>0$ minus the number of species with $r<0$ ), and a sink, if it has a net loss of species $(R<0)$, while neglecting immigration. The immigration of species is necessary to maintain both the species composition and interaction structure. Thus, for the network to persist, the rescue (Brown and KodricBrown, 1977) of individual species is essential. If some species go extinct, effects may cascade out to other parts of the local network, reducing the $r$ of other species (either directly or indirectly), and triggering further cascading extinctions (e.g. Palmer et al., 2008).

\subsection{Meta-networks and extinction}

From a meta-network perspective, extinction and colonisation can be envisaged on several organisational levels, for example, the interaction-, species-, local network-, meta-network-, local patch- and regional level. 
In an extreme case, an entire local patch might disappear, and with it the complete local network with its species and interactions.

As a local patch shrinks, some species and links will go extinct (Pauw, 2007; Rodríguez-Cabal et al., 2007), the consequences of which will depend on the network and ecosystem type. For instance, in antagonistic networks, mesopredator release (Crooks and Soulé, 1999) may trigger secondary extinctions. In contrast, in a mutualistic system, the loss of interactions could have negative effects on the immediate interaction partners, if there is limited functional redundancy among species (cf. Zamora, 2000). Since many species are taking part in both mutualistic and antagonistic interactions simultaneously (Fontaine et al., 2011), foreseeing the outcome of species loss on local networks is a challenging task.

Although reduction of habitat area does not always result in complete extinctions, it often reduces species abundances (Fahrig, 2003), with detrimental consequences for mutualistic partners (or consumers in food webs). A reduced abundance would, all else being equal, result in a reduced interaction frequency. Within pollination networks, this can lower plant fecundity (Pauw, 2007); in food webs, it can reduce predation pressure. Additionally, interactions might disappear if interaction partners are not lost but reduced to encounter probabilities approaching zero. Depending on whether the involved species have alternative partners, interaction extinction may lead to local species loss. If all local patches decrease sufficiently in area, the meta-network eventually fragments.

\subsection{Meta-networks and colonisation}

Both the abundance of the individual populations and the local species richness influence colonisation success. The more abundant and diverse the species are in the local habitat, the more difficult it is to colonise the local network, due to community closure, for instance (Hanski, 1999; MacArthur and Wilson, 1963). However, generality and competitiveness of the existing species and the area, isolation and quality of the local patch are also important factors (MacArthur and Wilson, 1963). Thus, the traits of both residents and colonists and fragment characteristics determine colonisation. For example, generalisation among the resident species may make it more difficult for colonists to find a vacant resource that is not already exploited. On the other hand, there may be many potential interaction partners, as predicted by the theory of preferential attachment (Barabási et al., 1999; Jordano et al., 2003; Olesen et al., 
2008). Thus, the effects of generalisation depend to a large extent on which community (mode) is exhibiting this trait (e.g. plant or pollinator trophic level in a bimodal network) and which is colonising.

It becomes more difficult to invade local networks that are characterised by a large number of generalist species, which might partly explain slow recovery of freshwater food webs from acidification (Layer et al., 2010, 2011). Networks consisting of many pairwise mutualistic interactions, for example, having tightly coevolved traits, might be more resistant to colonisation because species might be better able to compete for their resources.

The seminal work on island biogeography theory (MacArthur and Wilson, 1963) and later elaborated by other authors (e.g. Brown and KodricBrown, 1977; Whittaker et al., 2008) is especially relevant in this context: patches that are close to a source of dispersing species will, all else being equal, receive more colonisers and be less prone to extinction as they are more likely to be rescued (Brown and Kodric-Brown, 1977). As such, patches close to a source should therefore be better able to retain network structure than distant patches (of equal size).

As an additional consideration, bipartite ecological networks consisting of plant-pollinators or plant-seed dispersers contain both mutualistic and competitive interactions. On the one hand, plants and animals are involved in mutualistic interactions that might range from facultative to obligate, while pollinators interact competitively for resources (Goulson, 2003), as do some plants for pollinators (Morales and Traveset, 2009; Vamosi et al., 2006). Other plants do not compete (Hegland and Totland, 2008; Ollerton et al., 2003) or may even facilitate the pollinators of other species (Sargent et al., 2011). In cases of competition, the immigration, colonisation and extinction processes are governed by both antagonistic and mutualistic events depending on whether the interaction is related to similar nodes in the network. As a consequence, the simultaneous integration of both antagonistic and mutualistic network models (Klausmeier, 2001; Nee et al., 1997) might be needed. This will dramatically complicate any modelling process, especially when dealing with ecological networks of natural sizes (in a database of 54 communitywide pollination networks, species richness ranges from 16 to 952 species with a median of 105; Trøjelsgaard and Olesen, in press and similar-sized food webs are listed in Ings et al., 2009). Like extinction probability, colonisation ability will depend on species traits, including body size, 
mobility and generality. Generalists are often considered relatively good colonisers (Hanski, 1999), as are larger animals (Hoekstra and Fagan, 1998; Lomolino, 1985; Sutherland et al., 2000).

\section{EFFECTS OF HABITAT FRAGMENTATION ON THE
COEVOLUTIONARY DYNAMICS OF NETWORKS}

So far we have focused upon the ecological consequences of habitat fragmentation, while only briefly touching on evolutionary processes. For example, we implicitly assume that a species is more likely to die out due to the loss of its mutualistic partners or prey instead of natural selection leading to rapid evolution of new interactions (e.g. Rezende et al., 2007). However, there is increasing evidence that human-driven evolutionary change can occur on very short ('ecological') time scales (Darimont et al., 2009), which has implications for ecological networks. A first step in this direction might be to use the geographic mosaic theory of coevolution (GMTC) (Thompson, 2005) to describe how selection will vary across fragmented landscapes and how that might influence species interactions and ecological networks.

\subsection{The geographic mosaic theory of coevolution}

The GMTC assumes that the evolutionary dynamics of species interactions are affected by the spatial configuration of potentially interacting populations (Thompson, 2005). GMTC models assume that (i) species interact in discrete habitat patches, (ii) selective pressures associated with interactions vary across space (hereafter geographic selective mosaics) and (iii) gene flow mixes traits among populations (Gomulkiewicz et al., 2000; Nuismer and Doebeli, 2004; Nuismer and Thompson, 2006; Nuismer et al., 1999, 2000).

Geographical mismatches among potentially interacting species, geographically selective mosaics and gene flow will lead to unique evolutionary dynamics that cannot be predicted by single-site models. Space is a key component of this theory, affecting evolutionary dynamics in three ways. First, geographical variation in genotype distributions among populations will alter fitness. Second, space generates geographic selective mosaics where there is spatial variation in the function that connects the fitness of genotype in one species with that of its interacting partner. The geographic selective mosaics occur if the fitness and, consequently, selective pressures 
are determined by an interaction of two genotypes $(G)$ and by the environment $(E)$ (i.e. $G \times G \times E$ ). Third, the spatial configuration of sites will affect gene flow across populations (Nuismer et al., 2000).

\subsection{Habitat fragmentation and its effects on basic components of GMTC}

Habitat fragmentation could affect the GMTC for two-species interactions through its basic components: the patches, species interactions, gene flow and by changing the environment in which the interactions occur. The resulting poorly connected patches will be smaller than natural patches. The within-patch variation will increase due to contrasting selection and stochastic genetic variation in the many fragmented subpopulations of a given species. In this sense, the unique (biotic) history of each fragment might lead to an equally unique combination of abiotic factors that might affect the selective pressures on the interaction.

If the landscape is perceived by a given species as a composition of isolated fragments, a break-up of interactions in some patches is expected. For instance, the local extinction of some top predators in rainforest fragments can lead to the loss of key predator-prey interactions that can affect the whole ecosystem via trophic cascades (Terborgh et al., 2001). The same is true for some large frugivores, whose extinction may lead to the loss of key interactions with large-seeded plants (Guimarães et al., 2008). On the other hand, new interactions could also be created by invasive species that might be able to persist in the fragments but not in the original connected environment, as open-habitat species may eventually use secondary forest fragments or species that were present before fragmentation 'rewire' their interactions due to some interacting partner loss. At present, the consequences of losing (or gaining) such key species on the selective pressures associated to interactions remain virtually unknown from a fragmentation perspective.

Habitat fragmentation could also alter the relevance of certain interactions, via changes in abundances of interacting species. Species abundance shapes ecological networks and common species are often also highly connected (e.g. Krishna et al., 2008). Changes in abundance due to fragmentation may, in turn, affect the selective pressures associated with particular interactions. An additional related factor is the reduction of gene flow across patches, which might ultimately have major consequences on species evolution and coevolution (Nuismer et al., 1999). Mathematical models of GMTC suggest that gene flow can have unexpected evolutionary consequences for local adaptation in pairwise interactions (Nuismer et al., 1999). 


\subsection{Habitat fragmentation and selection mosaics in ecological networks}

Examining the GMTC in a fragmentation setting is especially challenging in species-rich networks, because the dynamical consequences of network structure are not simply the sum of the dynamics of pairwise interactions. For instance, if a network has $N$ species in a continuous habitat, there are $2^{N}$ possible combinations of species for any given habitat fragment. Again, simplification is the route to address this challenge and we need to learn first more about which are the most relevant components of ecological networks to understand how they affect the speed and direction of evolutionary change.

Recent studies are starting to explore the role of species ecological networks in shaping evolutionary dynamics. For instance, ecological networks of interacting species might favour the maintenance of high levels of trait diversity (Fontaine et al., 2011). Explorations of the evolutionary dynamics in species ecological networks by integrating field data, evolutionary models and tools derived from statistical mechanics are still in their infancy. In mutualistic networks, evolutionary dynamics appear to be shaped mainly by a few super-generalist species that interact with multiple modules (Olesen et al., 2007). Such species shape the evolution and coevolution in these networks in multiple ways (Guimarães et al., 2011). First, they increase the frequency of evolutionary cascades through a small-world effect, by reducing path length between species within the network. Second, they create asymmetric dependencies among species, reducing the potential of reciprocal selection. Third, they impose similar selective pressures over multiple components of the network, promoting convergence in species traits (Guimarães et al., 2011). The hypothesised effects of super-generalists provide the first steps in predicting the potential evolutionary consequences of habitat fragmentation in ecological networks.

Changes in species composition will be particularly relevant if supergeneralists are affected. For instance, the probability of local extinction increases with body size (Gaston and Blackburn, 1995), which is itself often positively associated with generalisation in both antagonistic predator-prey and mutualistic seed dispersal interactions. Thus, size-based extinctions are more likely to lead to the extinction of super-generalists and this could conceivably lead to an increase in the role of reciprocal selection. Furthermore, it could reduce the frequency of evolutionary cascades, ultimately favouring trait dissimilarity (i.e. mismatches) within interacting assemblages. In contrast, the introduction of generalist exotic species, such as honeybees, may 
favour convergence among plants (Guimarães et al., 2011). Therefore, habitat fragmentation may change the evolutionary dynamics within species networks, especially if super-generalists die out or invade newly fragmented habitats.

If the degree of habitat loss and fragmentation leads to a set of very small and disconnected fragments, each should have tiny and semi-autonomous networks with little dispersal among them (Fig. 20). These networks would be unlikely to contain super-generalist species that rely upon a diversity of partners to survive. Species that specialise on a few partners, such as largeseeded plants that use large vertebrates for dispersal, will also be absent (Da Silva and Tabarelli, 2000). Consequently, these tiny networks should contain species with relatively homogeneous interaction patterns, with no one species dominating evolutionary or coevolutionary processes in the network. Moreover, divergence in population traits due to local adaptation may occur if these small networks are also isolated. Finally, the role of species across networks is not fixed, although we still know little about this (but see Marquitti, 2011). Changing the abiotic and biotic features in a given patch, habitat fragmentation could alter both the ecology and evolution of interacting species. For example, forest fragmentation might suppress the population of a super-generalist species, transforming it to a peripheral species in the network and consequently reducing its ecological relevance and as well affecting evolutionary trajectories within the entire community.

Predicting evolutionary consequences of fragmentation on networks is still limited by a relative lack of both data and a mature theoretical framework. Theoretical studies using two-species models suggest that the coevolutionary dynamics may be qualitatively changed because of gene flow (Nuismer et al., 1999), and the potential for new evolutionary dynamics is even higher in a species-rich and fragmented network. The challenge ahead is to develop approaches to model these complex dynamics in ways that allow hypotheses to be tested in the field.

\section{APPLICATIONS IN CONSERVATION AND AGRICULTURE}

The effects of habitat loss and fragmentation on biodiversity are evident on a global scale, and researchers and managers must develop ways to understand and mitigate them (Bazelet and Samways, 2011). For instance, many European bird species have declined as agricultural intensification has resulted in the increasing fragmentation and isolation of natural habitats (Donald et al., 2001; Tscharntke et al., 2005), and yet the consequences of 
losing these often key species from mutualistic or antagonistic networks are still largely unknown. What is clear, however, is that the effects of habitat fragmentation are not evenly distributed within or among networks (e.g. Cagnolo et al., 2009).

The growing appreciation that the importance of network structure for ecosystem stability and functioning recognises that it is linked intrinsically to applied goals, such as biodiversity conservation (Kaiser-Bunbury et al., 2010; Tylianakis et al., 2010) or agricultural production (e.g. MacFadyen et al., 2011). Yet for network approaches to become fully integrated into ecosystem management, two objectives must be met. First, a conceptual challenge will be to demonstrate that complex network approaches add value to current practices. Underpinning this is the need to identify which specific attributes of networks require the greatest attention and which offer the best yield-to-effort reward. Second, a variety of practical hurdles need to be overcome, both in the quantification of network attributes using empirical data that can be feasibly obtained and in the application of concepts to practice (Tylianakis et al., 2010).

Gathering conceptual support for the adoption of network tools is the easier of these two objectives. The importance of network structure for properties such as system stability, and recognition that this can be altered even when species richness is not (e.g. Tylianakis et al., 2007), suggests that landscape degradation may be altering ecosystems in ways that cannot be detected by simple species-centric measurements. Furthermore, species cannot survive without their interacting partners, so there is an inherent need to consider the resources and mutualists of any species we wish to conserve. Additionally, the extinction sequence of species and interactions from a network during the fragmentation process (e.g. Sabatino et al., 2010) could provide guidance on the order in which species should be (re)introduced during restoration (Feld et al., 2011). A network perspective can also help predict the indirect effects of species additions or deletions (Carvalheiro et al., 2008). A major challenge now is to identify the most relevant aspects of network architecture for agriculture and conservation within fragmented landscapes, whilst taking into account the huge complexity of these networks.

One promising avenue in this context is to focus on some key components (e.g. species, links, functional roles, modules), as identified via network analysis, that are needed for the system to function 'normally'. For example, evidence is growing that super-generalists are the backbone of many networks, potentially governing their ecological and evolutionary dynamics (Guimarães et al., 2011; Olesen et al., 2007), which could provide clues as to how best to conserve or restore fragmented landscapes. There is also plenty of evidence 
that top predators can have cascading effects in marine, terrestrial and freshwater ecosystems worldwide (Estes et al., 2011), and many of these are also highly generalised. The reintroduction of locally extinct generalists may assist the restoration of previous ecosystem states whereas the removal of non-native super-generalists may be the first step needed to restore fragments and landscapes to their prior condition.

In addition to the presence or absence of apex consumers and supergeneralists, several other network metrics can be important from a conservation perspective. Tylianakis et al. (2010) argued that conservation could focus on network attributes that confer stability or maximise rates of ecosystem functioning. Nestedness, compartmentalisation, degree distributions, interaction diversity and the presence of weak links are all potentially useful metrics, but some of these are sensitive to sampling effort. Thus, the best approach to conservation of complex networks could involve the monitoring and/or restoration of a suite of network metrics, at least if preserving stability and functioning are the primary objectives (Tylianakis et al., 2010). These would likely include measures of connectedness (such as connectance or link density), which would relate to functional redundancy and the probability of secondary extinctions following species loss. Furthermore, compartmentalisation or modularity (particularly to avoid the spread of pollutants or perturbations) and nestedness (to maintain robustness of functioning following local extinctions) are likely to be key network properties for restoration and conservation.

Despite being important in theory, measuring network metrics accurately and manipulating them empirically remains a hurdle to the implementation of a more 'link-focused' management. Simulations of sampling can help reveal which metrics may be least sensitive to sampling effort (Nielsen and Bascompte, 2007; Tylianakis et al., 2010), and these may be the optimal candidates for biomonitoring. A number of questions still need to be addressed before network conservation can be put into practice. At the most basic level, we need to know how the survival or conservation of a species in a fragmented landscape is affected by its biotic context, that is, the number and kinds of links connecting that species to others within the network. Second, we need to identify the traits of species that determine their role within the network, so that we can begin to predict and restore network structure. For example, species traits such as body size and morphology (e.g. Stang et al., 2007, 2009; Woodward et al., 2005) are known to influence network structure, and techniques have recently been developed to calculate the contribution of a species to network nestedness 
and persistence (Saavedra et al., 2011). As ecologists further unravel these traits, we can start to move towards developing a predictive framework for network architecture given community-wide traits of species (Gilljam et al., 2011; Petchey et al., 2008; Woodward et al., 2010b). Third, we need to better understand the relationship between physical structure and network architecture. Evidence that complex habitat structures can impede the realisation of potential interactions (Laliberté and Tylianakis, 2010) requires consideration in the restoration of complex (e.g. forest) habitats and provides a potential avenue for reducing the impact of undesirable or strong destabilising interactions.

\section{CONCLUSIONS}

Our synthesis provides ample evidence that the consequences of habitat fragmentation for biotic communities and ecological networks are highly complex, but that does not mean they are unpredictable. At least five components of this complexity become immediately apparent. First, there is spatial complexity in the fragmentation process due to variation in landscape structure in terms of fragment size and isolation, connectivity, matrix quality, edge permeability and geometry. Second, fragmentation can affect the temporal dynamics of interacting taxa (e.g. flowering and fruiting phenologies), and long-term consequences on interacting species may become apparent only after several decades. For instance, time lags will increase the probability of co-extinctions, especially when generation times strongly differ between interacting taxa. Third, responses by fragmentation- and network-relevant traits differ among species. The perception of fragmentation (e.g. environmental grain) by individual species, key traits and complexes (e.g. body size in food webs), and trait matching between interacting species might be particularly relevant for assessing the consequences of fragmentation. Fourth, there is complexity in the biological and analytical details of networks, which differ in type (e.g. mutualistic vs. antagonistic; bimodal vs. multi-modal). Effects of dispersal, colonisation and extinction need to be integrated (e.g. in meta-networks). Fifth, there is an evolutionary component to network responses to habitat fragmentation. The geographic settings of habitat configuration and selective mosaics might lead to rapid evolutionary changes, even at short 'ecological' time scales. Finally, these five complexity components may interact, creating potential synergies. 
How can we usefully address and simplify this extreme complexity that originates from different spatial and temporal scales and organisational levels? First, we need to understand how individual links among interacting species are affected by habitat fragmentation, both in a spatial and temporal setting. These include phenologies and encounter rates and how they vary across space, time and levels of fragmentation. Second, there is overwhelming evidence that species are not equally important for ecosystem functioning and that a few exert disproportionate effects. These include large species at high trophic levels (e.g. top predators), abundant species and super-generalists. Such species can provide the structural backbones of ecological networks, shape evolutionary dynamics or initiate cascades of network changes. Thus, one way to circumvent the apparent complexity is to focus initially on understanding how fragmentation affects these key species and their links. Third, we need to gauge the extent of functional redundancy in ecological networks and to what extent habitat fragmentation disproportionally affects functionally unique species. This includes a better understanding of the role of specialisation, functional grouping and trait matching in ecological networks. Finally, we need to understand in more detail how network properties (e.g. connectance, linkage level, nestedness, modularity) and the roles of species in networks (e.g. hubs, connectors, spatial couplers) are affected by habitat fragmentation. This will become particularly interesting as we begin to link different types of networks, for example, when combining spatial with ecological networks or when moving from simple networks to meta- and super-networks.

There is a clear need to consider ecological and evolutionary processes of multispecies interactions in a network context to understand how habitat fragmentation affects biodiversity. Such an approach will become increasingly feasible as the availability of large databases, appropriate software and comparative studies continue to increase apace. We envisage a hierarchical approach to understand how individuals, populations, pairwise interactions, ecological networks and ultimately networks of networks are affected by fragmentation. For network approaches to become integrated into conservation, agriculture and ecosystem management, we need to find ways to simplify the inherent complexity and to measure and monitor management-relevant network properties. A link-based management approach has great potential to aid biodiversity conservation and restoration by highlighting the immense importance of biotic interactions and ecological network stability for ecosystem functioning. 


\section{ACKNOWLEDGEMENTS}

This paper was developed and written during and after two workshops sponsored by the Danish Agency for Science, Technology and Innovation (FNU) under the international network call (Application title: 'Ecological Network Analysis in an Agricultural Landscape'). In addition, we acknowledge support from the Danish Council for Independent Research | Natural Sciences (M. H.; J. M. O.; and via a starting independent researcher grant (11-106163) to W. D. K.), the Carlsberg Foundation (C. R.), the Villum Kann Rasmussen Foundation (W. D. K., VKR09b-141 to J.- C. Svenning), the Brazilian Council for Science CNPq (M. A. M. D. A., L. P. C. M.) and the Swiss National Science Foundation (C. N. K. -B.). M. A. M. D. A., J. G., P. R. G., F. M. D. M., K. P. M., L. P. C. M. and M. M. V. were partially supported by Fundação de Amparo a Pesquisa do Estado de São Paulo (FAPESP), J. M. T was funded by a Rutherford Discovery Fellowship, and M. M. V. was partially supported by CAPES. G. B. J. and E. J. O. were supported by UK Natural Environment Research Council grants awarded to G. W. (Ref: NE/I528069/1; NE/I009280/1). We are most grateful to C. Mulder for his many corrections and comments.

\section{APPENDIX}

\section{Methods for Ashdown Forest case study of food webs in fragmented river networks}

\section{A.1 Site description and food web construction}

Ashdown Forest in Sussex, UK (National Grid Reference TQ 520300) contains the spring-fed headwaters of two rivers, the Ouse-which flows south into the English Channel-and the Medway, which flows north and joins the Thames estuary. The catchments of both streams lie in the centre of the Weald in SE England, on hills of soft, fine sandstone (Ashdown Sands). Further description of the site can be found in Townsend et al. (1983). Sixteen streams were sampled in this study, and $\mathrm{pH}$ was recorded in 1976 and 1994, and an average value was calculated for each stream. Five randomly dispersed Surber samples (sample-unit area $0.0625 \mathrm{~m}^{2}$; mesh aperture $330 \mu \mathrm{m}$ ) were collected from each of the 16 streams in October 1976, 1984 and 1994 (Gjerløv et al., 2003; Townsend et al., 1987) (total $n$ sampleunits $=240$ ). The benthos was disturbed to a depth of approximately $5 \mathrm{~cm}$ and all macroinvertebrates collected were preserved in the field and subsequently sorted. Taxonomic identification was standardised to the highest common level of resolution (usually to species) across all webs (Woodward et al., 2002a). Several of the more difficult to identify taxa were aggregated: for example, all members of the Tanypodinae subfamily were presented as a single node. Feeding links were taken from 
direct observed interactions (gut contents analysis) in Broadstone Stream (Woodward et al., 2010b) and elsewhere within these two river networks (e.g. Layer et al., 2010, 2011), and this dataset was augmented with feeding links inferred from known interactions described in the literature from different systems (Brose et al., 2006; Gilljam et al., 2011; Lancaster et al., 2005; Warren, 1996; Woodward et al., 2008, 2010b). Additional feeding link data were supplied by F. Edwards (unpublished data).

\section{GLOSSARY}

Note that some of the terms in this glossary have alternate meanings, and some also have general and specific definitions (e.g. complexity) in different disciplines (e.g. in food webs vs. mutualistic networks; in landscape ecology vs. ecological network ecology), which can lead to potential misunderstandings when undertaking interdisciplinary research. We have highlighted these with ‘ $\star$ ', below.

Antagonistic network (p. 96) A network with associations between organisms in which one benefits at the expense of the other, for example, food webs, host-parasitoid networks and competitive networks.

^Asymmetry (p. 199) In a network context, a property of nested assemblages (e.g. mutualistic networks). Specialist plants interact just with generalist animals, while generalist animals use a broad range of host plants, including both specialists and generalists. It also refers to inequality of strong and weak interactions between species or nodes, competition or energy flow within a network.

Bimodal networks (p. 97) Pollination and seed dispersal networks are by definition bimodal (bipartite or two-mode), linking two sets of taxa (e.g. flower-visitors and plants, or frugivores and plants). They are often best represented by two-level bipartite graphs. Host-parasitoid networks or food webs that consider just two trophic levels also fall under this definition.

Boundary (p. 117) A border (or edge) between contrasting habitat patches that delimits the spatial heterogeneity of a landscape.

Centrality (p. 102) A measure of the importance of a node as a focal point within a network. There are various types of centrality measures for any node within a network, such as degree (the number of nodes that a focal node is connected to), closeness (the inverse sum of shortest distances to all other nodes from a focal node) and betweenness (the degree to which a node lies on the shortest path between two other nodes).

Coevolutionary dynamics (p. 91) Coevolution is the process of reciprocal evolutionary change between interacting species, driven by natural selection. This may lead to coevolutionary dynamics, whereby changes in gene frequency in one species trigger reciprocal changes in the other interacting species.

Compartment (p. 97) An assemblage of species within a network. Specific definitions vary depending on the point of view of the constituent organisms. Density view: an assemblage of species that are highly connected to each other. Predator view: an assemblage of species that share a large number of prey. Prey view: an assemblage of species that share a large number of predators. See also module below. 
Compartmentalisation (p. 98) The development of groups of species or 'topological compartments' that have a higher probability of interacting with one another than with other species in the network. See also modularity below.

*Complexity (p. 92) Property or set of properties that characterise systems composed of many interacting parts or elements. In organised complexity, the non-random or correlated interaction among the parts generates emergent properties, that is, properties not carried or dictated by individual parts. In ecology, complexity can be used as a general term (to describe a large number of interacting nodes) or with a more specific definition, for example, the average number of trophic links per species within a whole food web.

Connectance (p. 91) The proportion of all possible interactions within a system that are realised. This is typically measured as directed connectance, the proportion of documented directed links out of the maximum number of possible directed links in the food web, that is, the number of links $(L)$ divided by the number of species $(S)$ squared, $L / S^{2}$.

Connectors (p. 143) Species that link different modules within a network together. For example, large-bodied species, which disperse widely and thus link subwebs together (e.g. avian predators in a fragmented landscape).

Corridor (p. 92) Long, thin strips of habitat that connect otherwise isolated habitat patches. They reduce local extinction risk by connecting isolated populations and by promoting gene flow.

Degree distribution/linkage level distribution (p. 97, 174) The probability distribution of the number of links per node, typically measured over an entire network.

Domatium(-a) (p. 98) Specialised chamber(s) in different plant parts, providing refuge for predatory arthropods.

Ecological network (p. 91) A representation of biotic interactions in a multispecies community, in which pairs of species or other forms of taxonomic or functional aggregates (nodes) are connected when they are interacting (links), both directly and indirectly (e.g. sharing the same resource but not directly linked). There are three broad categoriesfood webs, host-parasitoid and mutualistic networks.

^Edge (p. 90) In a landscape context, the (artificial) boundaries of habitat fragments. Also used as a synonym for link in network analysis, highlighting the need for clarity when using this term in interdisciplinary studies.

Edge permeability (p. 103) The extent to which a species can move through a physical border, for example, from a fragment to the surrounding matrix. A 'hard' edge contains an impenetrable boundary which dispersing individuals virtually never cross, for example, a physical barrier such as an ocean surrounding an island. A 'soft' edge is more permeable to emigrating individuals than a hard edge, for example, the boundary between a meadow and a garden.

Environmental grain (p. 91) The scale of environmental variation (temporal or spatial), relative to the temporal/spatial scales of activity of the organisms, that is, a description of the organism's 'perception' of its own environment.

Fragments (p. 92) Habitat that was once continuous but has become divided into discrete patches. Fragments are separated by and embedded within areas (matrix) with abiotic and biotic properties different from the previously continuous habitat (see habitat fragmentation below).

Functional group (p. 94) A group of species or taxa with a similar response to a given factor. This may also include trophic species, groups of taxa that share the same set of predators and prey. 
Functional redundancy (p. 91) The idea that some species perform similar roles in communities and ecosystems and may therefore be substitutable with little impact on system properties.

Generalist (p. 98) A species that is able to thrive in a wide variety of environmental conditions and/or can make use of a variety of different resources.

Habitat fragmentation (p. 90) A process during which a large expanse of habitat is transformed into a number of smaller patches of smaller total area, isolated from each other by a matrix of habitats unlike the original. The effects of this process may include some, but not all of the following: (1) reduction in habitat amount, (2) increase in number of habitat patches, (3) decrease in size of habitat patches and (4) increase in isolation of patches.

Higher trophic rank hypothesis (p. 159) Species found at higher trophic levels tend to have a stronger relationship with area than species found at lower trophic levels as they have larger space and resource requirements. As such, species found at high trophic levels should have a higher susceptibility towards habitat fragmentation.

Host-parasitoid networks (p. 92) A specific form of antagonistic ecological network in which parasitoids benefit and subsist off their hosts. They may also contain information about hyperparasitoids (parasitoids that attack other parasitoids). These networks often involve a high degree of specialisation.

Hub (p. 91) Highly linked species within their own module of a network.

Interaction intimacy (p. 132) Degree of biological association between individuals of interacting species, for example, host-parasite during all of their life or only part of their lifespan.

*Interaction strength (p. 134) The magnitude of the effect of one species on another mediated by their pairwise interaction. This can be measured in a variety of ways, including experimental and theoretical approaches, or using allometric body-size scaling relationships.

Invasive species (p. 117) Species that arrive, become established and subsequently disperse in a community where they did not previously exist in historical time.

Link (p. 91) The pairwise interaction between two nodes in a network.

Linkage level (p. 97) Number of links per species.

*Matrix (p. 91) A landscape that has undergone fragmentation, often leading to a heterogenous habitat. Also quantifies the pairwise interactions between multiple species in a network, for example, qualitative (presence/absence of an interaction) or quantitative (coefficients reflecting interaction strengths, such as the Community or Jacobian matrix). The different meanings of this term in different fields of ecology highlight the importance of clarity in interdisciplinary studies.

Matrix permeability (p. 118) The property of a habitat matrix that describes the extent to which species can move through it, that is, between fragmented habitat patches.

Meta-populations/meta-communities (p. 101, 164) Potentially unstable local populations inhabiting discrete habitat patches, which persist at a larger scale via dispersal.

Module/modularity (p. 91) Ecological networks consist of link-dense and link-sparse areas. Link-dense regions are termed compartments or modules. Species within a module are linked more tightly together than they are to species in other modules. The extent to which species interactions are organised into modules is termed the modularity of the network. Modularity may reflect habitat heterogeneity, divergent selection regimes and phylogenetic clustering of closely related species.

Mutualistic networks (p. 96) Networks where both groups benefit from each other. Examples include plant-animal interactions (typically pollinators, frugivores, ants), 
plant-mycorrhizal systems, coral-zooxanthellae associations and many other networks involving microbial endosymbionts. These networks do not exist on multiple trophic levels, unlike antagonistic networks.

^Nestedness (p. 91) A distinctive pattern of mutualistic community assembly showing two characteristics, namely, asymmetrical specialisation (specialists interacting with generalists) and a generalist core (generalists interacting with generalists). Nestedness occurs when specialist species interact with a proper subset of species with which more generalised species interact. Nestedness can also describe niche overlap in antagonistic networks: for instance, where predator diets are arranged hierarchically on the basis of body size in food webs.

Nodes (p. 94) In an ecological network, nodes mostly refer to species or trophic groups of species. In a broader context, however, nodes can also refer to individuals, populations, functional groups (e.g. body-size or feeding groups), guilds, communities or even entire networks.

Sink (p. 135) A habitat in which mortality exceeds production and is reliant on immigration to maintain population levels.

Spatial network (p. 94) A network, or weighted spatial graph, where the nodes have a location and the links have lengths and also a magnitude or weight.

Specialist (p. 98) A species that can only thrive in a narrow range of environmental conditions and/or has a limited diet.

Super-generalist (p. 91) Species with a very high level of generalisation compared to coexisting species. In a network context, they will have a much higher linkage level and centrality than the other species. They are often super-abundant, density-compensating island species.

Super-network (p. 176) Expanding the network study from looking at single bipartite networks to multiple bipartite networks (e.g. plant-pollinator, plant-herbivore and plant-pathogen networks).

Topological role (peripherals, connectors, module and network hubs) (p. 101) Functional role of a node in the network in relation to the modular structure.

\section{REFERENCES}

Abramsky, Z., Tracy, C.R., 1979. Habitat selection in two species of short-horned grasshoppers. Oecologia 38, 359-374.

Abramsky, Z., Tracy, C.R., 1980. Relation between home range-size and regulation of population size in Microtus ochrogaster. Oikos 34, 347-355.

Aguilar, R., Ashworth, L., Galetto, L., Aizen, M.A., 2006. Plant reproductive susceptibility to habitat fragmentation: review and synthesis through a meta-analysis. Ecol. Lett. 9, 968-980.

Aizen, M.A., Feinsinger, P., 1994a. Forest fragmentation, pollination and plant reproduction in a Chaco dry forest, Argentina. Ecology 75, 330-351.

Aizen, M.A., Feinsinger, P., 1994b. Habitat fragmentation, native insect pollinators and feral honey-bees in Argentine Chaco Serrano. Ecol. Appl. 4, 378-392.

Aizen, M.A., Feinsinger, P., 2003. Bees not to be? Responses of insect pollinator faunas and lower pollination to habitat fragmentation. In: Bradshaw, G.A., Marquet, P.A. (Eds.), How Landscapes Change: Human Disturbance and Ecosystem Fragmentation in the Americas. Springer-Verlag, Berlin, pp. 111-129.

Aizen, M.A., Morales, C.L., Morales, J.M., 2008. Invasive mutualists erode native pollination webs. PLoS Biol. 6, 396-403. 
Alarcón, R., Waser, N.M., Ollerton, J., 2008. Year-to-year variation in the topology of a plant-pollinator interaction network. Oikos 117, 1796-1807.

Alberti, L.F., Morellato, L.P.C., 2010. Variation on fruit production of Nectandra megapotamica (Lauraceae) trees on the edge and interior of a semideciduous forest: a case study. Naturalia (São José do Rio Preto) 33, 57-68.

Albrecht, M., Duelli, P., Schmid, B., Müller, C.B., 2007. Interaction diversity within quantified insect food webs in restored and adjacent intensively managed meadows. J. Anim. Ecol. 76, 1015-1025.

Allen, K.R., 1951. The Horokiwi Stream: a study of a trout population. N. Z. Mar. Dept. Fish. Bull. 10-10a, 1-231.

Almeida-Neto, M., Campassi, F., Galetti, M., Jordano, P., Oliveira-Filho, A., 2008. Vertebrate dispersal syndromes along the Atlantic forest: broad-scale patterns and macroecological correlates. Glob. Ecol. Biogeogr. 17, 503-513.

Amarasekare, P., 2000. Coexistence of competing parasitoids on a patchily distributed host, local vs. spatial mechanisms. Ecology 81, 1286-1296.

Andren, H., 1994. Effects of habitat fragmentation on birds and mammals in landscapes with different proportions of suitable habitat—a review. Oikos 71, 355-366.

Andrieu, E., Dornier, A., Rouifed, S., Schatz, B., Cheptou, P.-O., 2009. The town Crepis and the country Crepis: how does fragmentation affect a plant-pollinator interaction? Acta Oecol. 35, 1-7.

Araújo, S.B.L., de Aguiar, M.A.M., 2007. Pattern formation, outbreaks and synchronization in food chains with two and three species. Phys. Rev. E 75, 061908.

Araújo, E.D., Costa, M., Chaud-Netto, J., Fowler, H.G., 2004. Body size and flight distance in stingless bees (Hymenoptera: Meliponini): inference of flight range and possible ecological implications. Braz. J. Biol. 64 (3B), 563-568.

Arim, M., Berazategui, M., Barreneche, J.M., Ziegler, L., Zarucki, M., Abades, S.R., 2011. Determinants of density-body size scaling within food webs and tools for their detection. Adv. Ecol. Res. 45, 1-39.

Armbruster, P., Hutchinson, R.A., Cotgreave, P., 2002. Factors influencing community structure in a South American tank bromeliad fauna. Oikos 96, 225-234.

Artz, D.R., Waddington, K.D., 2006. The effects of neighbouring tree islands on pollinator density and diversity and on pollination of a wet prairie species, Asclepias lanceolata (Apocynaceae). J. Ecol. 94, 597-608.

Ashworth, L., Aguilar, R., Galetto, L., Aizen, M.A., 2004. Why do pollination generalist and specialist plant species show similar reproductive susceptibility to habitat fragmentation? J. Ecol. 92, 717-719.

Atmar, W., Patterson, B.D., 1993. The measure of order and disorder in the distribution of species in fragmented habitat. Oecologia 96, 373-382.

Bach, C.E., Kelly, D., 2004. Effects of forest edges on herbivory in a New Zealand mistletoe, Alepis flavida. N. Z. J. Ecol. 28, 195-205.

Baldissera, R., Ganade, G., 2005. Predação de sementes ao longo de uma borda de Floresta Ombrófila Mista e pasta-gem. Acta Bot. Bras. 19, 161-165.

Bankowska, R., 1980. Fly communities of the family Syrphidae in natural and anthropogenic habitats of Poland. Memorabilia Zoologica. 33, 3-93.

Barabási, A.L., Albert, R., Jeong, H., 1999. Mean-field theory for scale-free random networks. Physica A 272, 173-187.

Barbaro, L., van Halder, I., 2009. Linking bird, carabid beetle and butterfly life-history traits to habitat fragmentation in mosaic landscapes. Ecography 32, 321-333.

Bascompte, J., Jordano, P., Melián, C.J., Olesen, J.M., 2003. The nested assembly of plant-animal mutualistic networks. Proc. Natl. Acad. Sci. USA 100, 9383-9387.

Bazelet, C.S., Samways, M.J., 2011. Relative importance of management vs. design for implementation of large-scale ecological networks. Landsc. Ecol. 26, 341-353. 
Beattie, A.J., 1985. Ant pollination. In: Beck, E., Birks, H.J.E., Connor, E.F. (Eds.), The Evolutionary Ecology of Ant-Plant Mutualisms. Cambridge University Press, Cambridge, pp. 96-109.

Bell, S.S., Brooks, R.A., Robbins, B.D., Fonseca, M.S., Hall, M.O., 2001. Faunal response to fragmentation in seagrass habitats: implications for seagrass conservation. Biol. Conserv. 100, 115-123.

Bender, D.J., Fahrig, L., 2005. Matrix structure obscures the relationship between interpatch movement and patch size and isolation. Ecology 86, 1023-1033.

Bender, D.J., Contreras, T.A., Fahrig, L., 1998. Habitat loss and population decline: a metaanalysis of the patch size effect. Ecology 79, 517-533.

Bender, D.J., Tischendorf, L., Fahrig, L., 2003. Using patch isolation metrics to predict animal movement in binary landscapes. Landsc. Ecol. 18, 17-39.

Bennun, L., Njoroge, P., 1999. Important Bird Areas in Kenya. Nature Kenya, Nairobi.

Berens, D.G., Farwig, N., Schaab, G., Boehning-Gaese, K., 2008. Exotic guavas are foci of forest regeneration in Kenyan farmland. Biotropica 40, 104-112.

Berlow, E.L., Neutel, A.-M., Cohen, J.E., de Ruiter, P.C., Ebenman, B., Emmerson, M., Fox, J.W., Jansen, V.A.A., Jones, J.I., Kokkoris, G.D., Logofet, D.O., McKane, A.J., et al., 2004. Interaction strengths in food webs: issues and opportunities. J. Anim. Ecol. 73, 585-598.

Berlow, E.L., Dunne, J.A., Martinez, N.D., Stark, P.B., Williams, R.J., Brose, U., 2009. Simple prediction of interaction strengths in complex food webs. Proc. Natl. Acad. Sci. USA 106, 187-191.

Bierregaard, R.O., Lovejoy, T.E., Kapos, V., Dos Santos, A.A., Hutchings, R.W., 1992. The biological dynamics of tropical rainforest fragments. Bioscience 42, 859-866.

Blanche, K.R., Ludwig, J.A., Cunningham, S.A., 2006. Proximity to rainforest enhances pollination and fruit set in orchards. J. Appl. Ecol. 43, 1182-1187.

Blüthgen, N., Menzel, F., Hovestadt, T., Fiala, B., Blüthgen, N., 2007. Specialization, constraints and conflicting interests in mutualistic networks. Curr. Biol. 17, 341-346.

Blüthgen, N., Fründ, J., Vázquez, D.P., Menzel, F., 2008. What do interaction network metrics tell us about specialization and biological traits? Ecology 89, 3387-3399.

Bolnick, D.I., Amarasekare, P., Araújo, M.S., Bürger, R., Levine, J.M., Novak, M., Rudolf, V.H.W., Schreiber, S.J., Urban, M.C., Vasseur, D.A., 2011. Why intraspecific trait variation matters in community ecology. Trends Ecol. Evol. 26, 183-192.

Bommarco, R., Biesmeijer, J.C., Meyer, B., Potts, S.G., Poyry, J., Roberts, S.P.M., Steffan-Dewenter, I., Ockinger, E., 2010. Dispersal capacity and diet breadth modify the response of wild bees to habitat loss. Proc. R. Soc. B 277, 2075-2082.

Bommarco, R., Lundin, O., Smith, H.G., Rundlöf, M., 2012. Drastic historic shifts in bumble-bee community composition in Sweden. Proc. R. Soc. B 279, 309-315.

Bonada, N., Dolédec, S., Statzner, B., 2007. Taxonomic and biological trait differences of stream macroinvertebrate communities between Mediterranean and temperate regions: implications for future climatic scenarios. Glob. Change Biol. 13, 1658-1671.

Bond, W.J., 1994. Do mutualisms matter-assessing the impact of pollinator and disperser disruption on plant extinction. Philos. Trans. R. Soc. B 344, 83-90.

Bonin, M.C., Almany, G.R., Jones, G.P., 2011. Contrasting effects of habitat loss and fragmentation on coral-associated reef fishes. Ecology 92, 1503-1512.

Borrell, B.J., 2005. Long tongues and loose niches: evolution of euglossine bees and their nectar flowers. Biotropica 37, 664-669.

Bostrom, C., Jackson, E.L., Simenstad, C.A., 2006. Seagrass landscapes and their effects on associated fauna: a review. Estuar. Coast. Shelf Sci. 68, 383-403.

Boulton, A.J., 2003. Parallels and contrasts in the effects of drought on stream macroinvertebrate assemblages. Freshw. Biol. 48, 1173-1185. 
Boyle, S.A., Smith, A.T., 2010. Can landscape and species characteristics predict primate presence in forest fragments in the Brazilian Amazon? Biol. Conserv. 143, 1134-1143.

Brooks, T.M., Pimm, S.L., Collar, N.J., 1997. Deforestation predicts the number of threatened birds in insular southeast Asia. Conserv. Biol. 11, 382-394.

Brose, U., Jonsson, T., Berlow, E.L., Warren, P., Banašek-Richter, C., Bersier, L.F., Blanchard, J.L., Brey, T., Carpenter, S.R., Blandenier, M.F.C., Cushing, L., Dawah, H.A., et al., 2006. Consumer-resource body-size relationships in natural food webs. Ecology 87, 2411-2417.

Brotons, L., Monkkonen, M., Martin, J.L., 2003. Are fragments islands? Landscape context and density-area relationships in boreal forest birds. Am. Nat. 162, 343-357.

Brown, J.H., Kodric-Brown, A., 1977. Turnover rates in insular biogeography: effect of immigration on extinction. Ecology 58, 445-449.

Brown, L.E., Milner, A.M., 2012. Rapid retreat of glacial ice reveals invertebrate community assembly dynamics. Glob. Change Biol. http://dx.doi.org/10.1111/j.13652486.2012.02675.x.

Brown, L.E., Hannah, D.M., Milner, A.M., 2007. Vulnerability of alpine stream biodiversity to shrinking glaciers and snowpacks. Glob. Change Biol. 13, 958-966.

Brown, L.E., Edwards, F.K., Milner, A.M., Woodward, G., Ledger, M.E., 2011. Food web complexity and allometric scaling relationships in stream mesocosms, implications for experimentation. J. Anim. Ecol. 80, 884-895.

Buckley, Y.M., Anderson, S., Catterall, C.P., Corlett, R.T., Engel, T., Gosper, C.R., Nathan, R., Richardson, D.M., Setter, M., Spiegel, O., Vivian-Smith, G., Voigt, F.A., et al., 2006. Management of plant invasions mediated by frugivore interactions. J. Appl. Ecol. 43, 848-857.

Buckley, H.L., Miller, T.E., Ellison, A.M., Gotelli, N.J., 2010. Local- to continental-scale variation in the richness and composition of an aquatic food web. Glob. Ecol. Biogeogr. 19, 711-723.

Buldyrev, S.V., Parshani, R., Paul, G., Stanley, H.E., Havlin, S., 2010. Catastrophic cascade of failures in interdependent networks. Nature 464, 1025-1028.

Burgess, V.J., Kelly, D., Robertson, A.W., Ladley, J.J., 2006. Positive effects of forest edges on plant reproduction: literature review and a case study of bee visitation to flowers of Peraxilla tetrapetala (Loranthaceae). N. Z. J. Ecol. 30, 179-190.

Burkle, L.A., Alarcón, R., 2011. The future of plant-pollinator diversity: understanding interaction networks across time, space and global change. Am. J. Bot. 98, 528-538.

Burns, K.C., 2006. A simple null model predicts fruit-frugivore interactions in a temperate rainforest. Oikos 115, 427-432.

Burns, K.C., Lake, B., 2009. Fruit-frugivore interactions in two southern hemisphere forests: allometry, phylogeny and body size. Oikos 118, 1901-1907.

Byrnes, J.E., Reed, D.C., Cardinale, B.J., Cavanaugh, K.C., Holbrook, S.J., Schmitt, R.J., 2011. Climate-driven increases in storm frequency simplify kelp forest food webs. Glob. Change Biol. 17, 2513-2524.

Cagnolo, L., Valladares, G., Salvo, A., Cabido, M., Zak, M., 2009. Habitat fragmentation and species loss across three interacting trophic levels: effects of life-history and foodweb traits. Conserv. Biol. 23, 1167-1175.

Camargo, M.G.G., Souza, R.M., Reys, P., Morellato, L.P.C., 2011. Effects of environmental conditions associated to the cardinal orientation on the reproductive phenology of the cerrado savanna tree Xylopia aromatic (Annonaceae). An. Acad. Bras. Cienc. 83, 1007-1020.

Cane, J.H., 1987. Estimation of bee size using intertegular span (Apoidea). J. Kansas Entomol. Soc. 60, 145-147.

Cane, J.H., Sipes, S., 2006. Floral specialization by bees: analytical methods and a revised lexicon for oligolecty. In: Waser, N.M., Ollerton, J. (Eds.), Plant-Pollinator Interactions: 
From Specialization to Generalization. University of Chicago Press, Chicago, IL, pp. 99-122.

Carpenter, S.R., 1982. Stemflow chemistry, effects on population dynamics of detritivorous mosquitoes in tree-hole ecosystems. Oecologia 53, 1-6.

Carstensen, D.W., Olesen, J.M., 2009. Wallacea and its nectarivorous birds: nestedness and modules. J. Biogeogr. 36, 1540-1550.

Carstensen, D.W., Dalsgaard, B., Svenning, J.C., Rahbek, C., Fjeldså, J., Sutherland, W.J., Olesen, J.M., 2012. Biogeographical modules and island roles: a comparison of Wallacea and the West Indies. J. Biogeogr. 39, 739-749.

Carvalheiro, L.G., Barbosa, E.R.M., Memmott, J., 2008. Pollinator networks, alien species and the conservation of rare plants: Trinia glauca as a case study. J. Appl. Ecol. 45, $1419-1427$.

Carvalheiro, L.G., Veldtman, R., Shenkute, A.G., Tesfay, G.B., Pirk, C.W.W., Donaldson, J.S., Nicolson, S.W., 2011. Natural and within-farmland biodiversity enhances crop productivity. Ecol. Lett. 14, 251-259.

Castle, M.D., Blanchard, J.L., Jennings, S., 2011. Predicted effects of behavioural movement and passive transport on individual growth and community size structure in marine ecosystems. Adv. Ecol. Res. 45, 41-66.

Chalfoun, A.D., Thompson, F.R., Ratnaswamy, M.J., 2002. Nest predators and fragmentation: a review and meta-analysis. Conserv. Biol. 16, 306-318.

Chamberlain, S.A., Holland, J.N., 2009. Body size predicts degree in ant-plant mutualistic networks. Funct. Ecol. 23, 196-202.

Chamberlain, S.A., Kilpatrick, J.R., Holland, J.N., 2010. Do extrafloral nectar resources, species abundances and body sizes contribute to the structure of ant-plant mutualistic networks? Oecologia 164, 741-750.

Christianini, A.V., Oliveira, P.S., 2009. The relevance of ants as seed rescuers of a primarily bird-dispersed tree in the neotropical cerrado savanna. Oecologia 160, 735-745.

Clarke, C.M., Kitching, R.L., 1993. The metazoan food webs of six Bornean Nepenthes species. Ecol. Entomol. 18, 7-16.

Cohen, J.E., Schittler, D.N., Raffaelli, D.G., Reuman, D.C., 2009. Food webs are more than the sum of their tritrophic parts. Proc. Natl. Acad. Sci. USA 106, 22335-22340.

Coker, D.J., Pratchett, M.S., Munday, P.L., 2009. Coral bleaching and habitat degradation increase susceptibility to predation for coral-dwelling fishes. Behav. Ecol. 20, 1204-1210.

Coll, M., Schmidt, A., Romanuk, T., Lotze, H.K., 2011. Food-web structure of seagrass communities across different spatial scales and human impacts. PLoS One 6, e22591.

Cook, W.M., Lane, K.T., Foster, B.L., Holt, R.D., 2002. Island theory, matrix effects and species richness patterns in habitat fragments. Ecol. Lett. 5, 619-623.

Corbet, S.A., 2000. Butterfly nectaring flowers, butterfly morphology and flower form. Entomol. Exp. Appl. 96, 289-298.

Corlett, R.T., 1998. Frugivory and seed dispersal by vertebrates in the Oriental (Indomalayan) region. Biol. Rev. 73, 413-448.

Cox, P.A., 1983. Extinction of the Hawaiian avifauna resulted in a change of pollinators for the ieie, Freycinetia arborea. Oikos 41, 195-199.

Craig, J.L., Stewart, A.M., Douglas, M.E., 1981. The foraging of New Zealand honeyeaters. N. Z. J. Zool. 8, 87-91.

Cronin, J.T., 2003. Matrix heterogeneity and host-parasitoid interactions in space. Ecology 84, 1506-1516.

Cronin, J.T., 2004. Host-parasitoid extinction and colonization in a fragmented prairie landscape. Oecologia 139, 503-514.

Cronin, J.T., Haynes, K.J., Dillemuth, F., 2004. Spider effects on planthopper mortality: dispersal, and spatial population dynamics. Ecology 85, 2134-2143. 
Crooks, K.R., 2002. Relative sensitivities of mammalian carnivores to habitat fragmentation. Conserv. Biol. 16, 488-502.

Crooks, K.R., Soulé, M.E., 1999. Mesopredator release and avifaunal extinctions in a fragmented system. Nature 400, 563-566.

Culver, D.C., Beattie, A.J., 1978. Myrmecochory in Viola: dynamics of seed-ant interactions in some West Virginia species. J. Ecol. 66, 53-72.

Cunningham, S.A., 2000. Effects of habitat fragmentation on the reproductive ecology of four plant species in Mallee Woodland. Conserv. Biol. 14, 758-768.

D'Eça Neves, F.F., Morellato, L.P.C., in press. Efeitos de borda na fenologia de árvores em floresta semidecídua de altitude na Serra do Japi, SP. In: Vasconcellos Neto, J., Polli, P.R. (Eds.), Novos Olhares da Serra do Japi. Editora da Unicamp, Campinas.

Da Silva, J.M.C., Tabarelli, M., 2000. Tree species impoverishment and the future flora of the Atlantic Forest of northeast Brazil. Nature 404, 72-74.

Dahm, C.N., Baker, M.A., Moore, D.I., Thibault, J.R., 2003. Coupled biogeochemical and hydrological responses of streams and rivers to drought. Freshw. Biol. 48, 1219-1231.

Dale, M.R.T., Fortin, M.-J., 2010. From graphs to spatial graphs. Annu. Rev. Ecol. Syst. 41, 21-38.

Dalsgaard, B., Martín, A.M.G., Olesen, J.M., Timmermann, A., Andersen, L.H., Ollerton, J., 2008. Pollination networks and functional specialization: a test using Lesser Antillean plant-hummingbird assemblages. Oikos 117, 789-793.

Damschem, E.I., Haddad, N.M., Orrock, J.L., Tewksbury, J.J., Levey, D.J., 2006. Corridors increase plant species richness at larger scales. Science 313, 1284-1286.

Darimont, C.T., Carlson, S.M., Kinnison, M.T., Paquet, P.C., Reimchen, T.E., Wilmers, C.C., 2009. Human predators outpace other agents of trait change in the wild. Proc. Natl. Acad. Sci. USA 106, 952-954.

Darvill, B., Knight, M.E., Goulson, D., 2004. Use of genetic markers to quantify bumblebee foraging range and nest density. Oikos 107, 471-478.

Davidson, D.W., 1988. Ecological studies of neotropical ant gardens. Ecology 69, $1138-1152$.

Davies, K.F., Margules, C.R., Lawrence, J.F., 2000. Which traits of species predict population declines in experimental forest fragments? Ecology 81, 1450-1461.

Davies, K.F., Margules, C.R., Lawrence, J.F., 2004. A synergistic effect puts rare, specialized species at greater risk of extinction. Ecology 85, 265-271.

Dawideit, B.A., Phillimore, A.B., Laube, I., Leisler, B., Böhning-Gaese, K., 2009. Ecomorphological predictors of natal dispersal distances in birds. J. Anim. Ecol. 78, 388-395.

Debinski, D.M., Holt, R.D., 2000. A survey and overview of habitat fragmentation experiments. Conserv. Biol. 14, 342-355.

Devlaeminck, R., Bossuyt, B., Hermy, M., 2005. Inflow of seeds through the forest edge: evidence from seed bank and vegetation patterns. Plant Ecol. 176, 1-17.

Deza, A.A., Anderson, T.W., 2010. Habitat fragmentation, patch size, and the recruitment and abundance of kelp forest fishes. Mar. Ecol. Prog. Ser. 416, 229-240.

Diaz-Castelazo, C., Guimarães, P.R., Jordano, P., Thompson, J.N., Marquis, R.J., Rico-Gray, V., 2010. Changes of a mutualistic network over time: reanalysis over a 10-year period. Ecology 91, 793-801.

Dick, C.W., 2001. Genetic rescue of remnant tropical trees by an alien pollinator. Proc. R. Soc. B 268, 2391-2396.

Didham, R.K., Lawton, J.H., 1999. Edge structure determines the magnitude of changes in microclimate and vegetation structure in tropical forest fragments. Biotropica 31, 17-30.

Didham, R.K., Ghazoul, J., Stork, N., Davis, A.J., 1996. Insects in fragmented forests: a functional approach. Trends Ecol. Evol. 11, 255-260.

Didham, R.K., Kapos, V., Ewers, R.M., 2012. Rethinking the conceptual foundations of habitat fragmentation research. Oikos 121, 161-170. 
Do Carmo, R.M., Franceschinelli, E.V., da Silveira, F.A., 2004. Introduced honeybees (Apis mellifera) reduce pollination success without affecting the floral resource taken by native pollinators. Biotropica 36, 371-376.

Doak, D.F., Marino, P.C., Kareiva, P.M., 1992. Spatial scale mediates the influence of habitat fragmentation on dispersal success-implications for conservation. Theor. Popul. Biol. 41, 315-336.

Donald, P.F., Green, R.E., Heath, M.F., 2001. Agricultural intensification and the collapse of Europe's farmland bird populations. Proc. R. Soc. B 268, 25-29.

Donatti, C.I., Guimarães, P.R., Galetti, M., Pizo, M.A., Marquitti, F.M.D., Dirzo, R., 2011. Analysis of a hyper-diverse seed dispersal network: modularity and underlying mechanisms. Ecol. Lett. 14, 773-781.

Duarte, C.M., Chiscano, C.L., 1999. Seagrass biomass and production: a reassessment. Aquat. Bot. 65, 159-174.

Dudareva, N., Pichersky, E. (Eds.), 2006. Biology of Floral Scent. CRC Press, Boca Raton.

Dunne, J.A., Williams, R.J., Martinez, N.D., 2002. Network structure and biodiversity loss in food webs: robustness increases with connectance. Ecol. Lett. 5, 558-567.

Dupont, Y.L., Olesen, J.M., 2009. Ecological modules and roles of species in heathland plant-insect flower visitor networks. J. Anim. Ecol. 78, 346-353.

Dupont, Y.L., Hansen, D.M., Rasmussen, J.T., Olesen, J.M., 2004. Evolutionary changes in nectar sugar composition associated with switches between bird and insect pollination: the Canarian bird-flower element revisited. Funct. Ecol. 18, 670-676.

Dupont, Y.L., Trøjelsgaard, K., Olesen, J.M., 2011. Scaling down from species to individuals: a flower-visitation network between individual honeybees and thistle plants. Oikos 120, 170-177.

Dupré, C., Ehrlén, J., 2002. Habitat configuration, species traits and plant distributions. J. Ecol. 90, 796-805.

Duthie, C., Gibbs, G., Burns, K.C., 2006. Seed dispersal by weta. Science 311, 1575.

Dyer, L.A., Walla, T.R., Greeney, H.F., Stireman III, J.O., Hazen, R.F., 2010. Diversity of interactions: a metric for studies of biodiversity. Biotropica 42, 281-289.

Ecroyd, C., 1993. In search of the wood rose. For. Bird 1993, 24-28.

Eggleston, D.B., Etherington, L.L., Elis, W.E., 1998. Organism response to habitat patchiness: species and habitat-dependent recruitment of decapod crustaceans. J. Exp. Mar. Biol. Ecol. 223, 111-132.

Ellstrand, N.C., Elam, D.R., 1993. Population genetic consequences of small population size: implications for plant conservation. Annu. Rev. Ecol. Syst. 24, 217-242.

Elton, C.S., 1927. Animal Ecology. Macmillian, New York.

Elzinga, J.A., van Nouhuys, S., van Leeuwen, D.J., Biere, A., 2007. Distribution and colonization ability of three parasitoids and their herbivorous host in a fragmented landscape. Basic Appl. Ecol. 8, 75-88.

Emmerson, M.C., Raffaelli, D., 2004. Predator-prey body size, interaction strength and the stability of a real food web. J. Anim. Ecol. 73, 399-409.

Eshiamwata, G.W., Berens, D.G., Bleher, B., Dean, W.R.J., Böhning-Gaese, K., 2006. Bird assemblages in isolated Ficus trees in Kenyan farmland. J. Trop. Ecol. 22, 723-726.

Estes, J.A., Terborgh, J., Brashares, J.S., Power, M.E., Berger, J., Bond, W.J., Carpenter, S.R., Essington, T.E., Holt, R.D., Jackson, J.B.C., Marquis, R.J., Oksanen, L., et al., 2011. Trophic downgrading of planet Earth. Science 333, 301-306.

Evans, W.A., Johnston, B., 1980. Fish Migration and Fish Passage: A Practical Guide to Solving Fish Passage Problems. U.S. Forest Service, Washington, DC, 163pp.

Everard, M., 1996. The importance of periodic droughts for maintaining diversity in the freshwater environment. Freshw. Forum 7, 33-50. 
Ewers, R.M., Didham, R.K., 2006. Confounding factors in the detection of species responses to habitat fragmentation. Biol. Rev. 81, 117-142.

Fadini, R.F., Fleury, M., Donatti, C.I., Galetti, M., 2009. Effects of frugivore impoverishment and seed predators on the recruitment of a keystone palm. Acta Oecol. 35, 188-196.

Faegri, K., Pijl, L.V.D., 1979. The principles of Pollination Ecology. Pergamon Press, Oxford.

Fahrig, L., 2003. Effects of habitat fragmentation on biodiversity. Annu. Rev. Ecol. Syst. 34, 487-515.

Fall, A., Fortin, M.-J., Manseau, M., O’Brien, D., 2007. Spatial graphs: principles and applications for habitat connectivity. Ecosystems 10, 448-461.

Faria, D., Laps, R.R., Baumgarten, J., Cetra, M., 2006. Bat and bird assemblages from forests and shade cacao plantations in two contrasting landscapes in the Atlantic rainforest of southern Bahia, Brazil. Biodivers. Conserv. 15, 587-612.

Faria, D., Paciencia, M.L.B., Dixo, M., Laps, R.R., Baumgarten, J., 2007. Ferns, frogs, lizards, birds and bats in forest fragments and shade cacao plantations in two contrasting landscapes in the Atlantic forest, Brazil. Biodivers. Conserv. 16, 2335-2357.

Feinsinger, P., 1978. Ecological interactions between plants and hummingbirds in a successional tropical community. Ecol. Monogr. 48, 269-287.

Feld, C.K., Birk, S., Bradley, D.C., Hering, D., Kail, J., Marzin, A., Melcher, A., Nemitz, D., Pederson, M.L., Pletter Bauer, F., Pont, D., Verdonschot, P.F.M., et al., 2011. From natural to degraded rivers and back again: a test of restoration ecology theory and practice. Adv. Ecol. Res. 44, 119-210.

Fenster, C.B., Dudash, M.R., 2001. Spatiotemporal variation in the role of hummingbirds as pollinators of Silene virginica. Ecology 82, 844-851.

Finn, D.S., Rasanen, K., Robinson, C.T., 2010. Physical and biological changes to a lengthening stream gradient following a decade of rapid glacial recession. Glob. Change Biol. $16,3314-3326$.

Fischer, J., Lindenmayer, D.B., 2002. Treating the nestedness temperature calculator as a "black box" can lead to false conclusions. Oikos 99, 193-199.

Fishbein, M., Venable, D.L., 1996. Diversity and temporal change in the effective pollinators of Asclepias tuberosa. Ecology 77, 1061-1073.

Fisher, J., Stott, J., Law, B.S., 2010. The disproportionate value of scattered trees. Biol. Conserv. 143, 1564-1567.

Fitzpatrick, Ú., Murray, T.E., Paxton, R.J., Breen, J., Cotton, D., Santorum, V., Brown, M.F., 2007. Rarity and decline in bumblebees-a test of causes and correlates in the Irish fauna. Biol. Conserv. 136, 185-194.

Fleming, T.H., Breitwisch, R., Whitesides, G.H., 1987. Patterns of tropical vertebrate frugivore diversity. Annu. Rev. Ecol. Syst. 18, 91-109.

Fleury, M., Galetti, M., 2006. Forest fragment size and microhabitat effects on palm seed predation. Biol. Conserv. 131, 1-13.

Fonseca, G., Benson, P.J., 2003. Biodiversity conservation demands open access. PLoS Biol. 1, e46.

Fonseca, G.R., Ganade, G., 1996. Asymmetries, compartments and null interactions in an Amazonian ant-plant community. J. Anim. Ecol. 65, 339-347.

Fontaine, C., Dajoz, I., Meriguet, J., Loreau, M., 2006. Functional diversity of plantpollinator interaction webs enhances the persistence of plant communities. PLoS Biol. 4, 129-135.

Fontaine, C., Guimarães Jr., P.R., Kéfi, S., Loeuille, N., Memmott, J., van der Putten, W.H., van Veen, F.J.F., Thébault, E., 2011. The ecological and evolutionary implications of merging different types of networks. Ecol. Lett. 14, 1170-1181.

Fortuna, M.A., Bascompte, J., 2006. Habitat loss and the structure of plant-animal mutualistic networks. Ecol. Lett. 9, 281-286. 
Fortuna, M.A., Bascompte, J., 2008. The network approach in ecology. In: Valladares, F., Camacho, A., Elosegi, A., Gracia, C., Estrada, M., Senar, J.C., Gili, J.P. (Eds.), Unity in Diversity: Reflections on Ecology After the Legacy of Ramon Margalef. Fundación BBVA, Bilbao, pp. 371-393.

Fortuna, M.A., Gómez-Rodríguez, C., Bascompte, J., 2006. Spatial network structure and amphibian persistence in stochastic environments. Proc. R. Soc. B 273, 1429-1434.

Fortuna, M.A., Stouffer, D.B., Olesen, J.M., Jordano, P., Mouillot, D., Krasnov, B.R., Poulin, R., Bascompte, J., 2010. Nestedness versus modularity in ecological networks: two sides of the same coin? J. Anim. Ecol. 79, 811-817.

Fox, H.E., 2004. Coral recruitment in blasted and unblasted sites in Indonesia: assessing rehabilitation potential. Marine Ecol. Prog. Ser. 269, 131-139.

Fragoso, J., 1997. Tapir-generated seed shadows: scale-dependent patchiness in the Amazon rain forest. J. Ecol. 85, 519-529.

Fragoso, J., Silvius, K., Correa, L., 2003. Long-distance seed dispersal by tapirs increases seed survival and aggregates tropical trees. Ecology 84, 1998-2006.

Franklin, J.E., Forman, R.T.T., 1987. Creating landscape patterns by forest cutting: ecological consequences and principles. Landsc. Ecol. 1, 5-18.

Frost, M.T., Rowden, A.A., Attrill, M.J., 1999. Effect of habitat fragmentation on the macroinvertebrate infaunal communities associated with the seagrass Zostera marina L. Aquat. Conserv. 9, 255-263.

Fuchs, E.J., Lobo, J.A., Quesada, M., 2003. Effects of forest fragmentation and flowering phenology on the reproductive success and mating patterns of the tropical dry forest tree Pachira quinata. Conserv. Biol. 17, 149-157.

Galetti, M., Donatti, C., Pires, A., Guimarães, P., Jordano, P., 2006. Seed survival and dispersal of an endemic Atlantic forest palm: the combined effects of defaunation and forest fragmentation. Bot. J. Linn. Soc. 151, 141-149.

Galetti, M., Giacomini, H.C., Bueno, R.S., Bernardo, C.S., Marques, R.M., Bovendorp, R.S., et al., 2009. Priority areas for the conservation of Atlantic forest large mammals. Biol. Conserv. 142, 1229-1241.

Ganzhorn, J.U., Eisenbeiss, B., 2001. The concept of nested species assemblages and its utility for understanding effects of habitat fragmentation. Basic Appl. Ecol. 2, 87-95.

Garcia, D., Chacoff, N.P., 2007. Scale-dependent effects of habitat fragmentation on hawthorn pollination, frugivory, and seed predation. Conserv. Biol. 21, 400-411.

Gaston, K.J., 1988. Patterns in local and regional dynamics of moth populations. Oikos 53, $49-57$.

Gaston, K.J., Blackburn, T.M., 1995. Birds, body size and the threat of extinction. Philos. Trans. R. Soc. B 347, 205-212.

Gathmann, A., Tscharntke, T., 2002. Foraging ranges of solitary bees. J. Anim. Ecol. 71, 757-764.

Gehlhausen, S.M., Schwartz, M.W., Augspurger, C.K., 2000. Vegetation and microclimatic edge effects in two mixed-mesophytic forest fragments. Plant Ecol. 147, 21-35.

Genini, J., Morrellato, L.P.C., Guimarães Jr., P.R., Olesen, J.M., 2010. Cheaters in mutualism networks. Biol. Lett. 6, 494-497.

Gilbert-Norton, L., Wilson, R., Stevens, J.R., Beard, K.H., 2010. A meta-analytic review of corridor effectiveness. Conserv. Biol. 24, 660-668.

Gill, F.B., Wolf, L.L., 1975. Economics of feeding territoriality in Golden-winged sunbird. Ecology 56, 333-345.

Gilljam, D., Thierry, A., Edwards, F.K., Figueroa, D., Ibbotson, A.T., Jones, J.I., Lauridsen, R.B., Petchey, O.L., Woodward, G., Ebenman, B., 2011. Seeing double: size-based versus taxonomic views of food web structure. Adv. Ecol. Res. 45, 67-134.

Gilpin, M.E., Diamond, J.M., 1981. Immigration and extinction probabilities for individual species: relation to incidence functions and species colonization curves. Proc. Natl. Acad. Sci. USA 78, 392-396. 
Girao, L.C., Lopes, A.V., Tabarelli, M., Bruna, E.M., 2007. Changes in tree reproductive traits reduce functional diversity in a fragmented Atlantic forest landscape. PLoS One 2, e908.

Githiru, M., Lens, L., Bennur, L.A., Ogol, C., 2002. Effects of site and fruit size on the composition of avian frugivore assemblages in a fragmented Afrotropical forest. Oikos 96, $320-330$.

Gjerløv, C., Hildrew, A.G., Jones, J.I., 2003. Mobility of stream invertebrates in relation to disturbance and refugia: a test of habitat templet theory. J. N. Am. Benthol. Soc. 22, 207-223.

Gliwicz, J., 1980. Island populations of rodents: their organization and functioning. Biol. Rev. 55, 109-138.

Goldblatt, P., Manning, J.C., 2000. The long-proboscid fly pollination system in southern Africa. Ann. Mo. Bot. Gard. 87, 146-170.

Gómez, J.M., 2000. Effectiveness of ants as pollinators of Lobularia maritima: effects on main sequential fitness of the host plant. Oecologia 122, 90-97.

Gomulkiewicz, R., Thompson, J.N., Holt, R.D., Nuismer, S.L., Hochberg, M.E., 2000. Hot spots, cold spots, and the geographic mosaic theory of coevolution. Am. Nat. 156, 156-174.

Gonzalez, A., Rayfield, B., Lindo, Z., 2011. The disentangled bank: how loss of habitat fragments and disassembles ecological networks. Am. J. Bot. 98, 503-516.

Goulson, D., 2003. Effects of introduced bees on native ecosystems. Annu. Rev. Ecol. Syst. $34,1-26$.

Graham, C., 2002. Use of fruiting trees by birds in continuous forest and riparian forest remnants in Los Tuxtlas, Veracruz, Mexico. Biotropica 34, 589-597.

Graham, N.A.J., Wilson, S.K., Jennings, S., Polunin, N.V.C., Robinson, J., Bijoux, J.P., Daw, T.M., 2007. Lag effects in the impacts of mass coral bleaching on coral reef fish, fisheries, and ecosystems. Conserv. Biol. 21, 1291-1300.

Greenleaf, S.S., Williams, N.M., Winfree, R., Kremen, C., 2007. Bee foraging ranges and their relationship to body size. Oecologia 153, 589-596.

Griffin, J.N., Butler, J., Soomdat, N.N., Brun, K.E., Chejanorski, Z.A., Silliman, B.R., 2011. Top predators suppress rather than facilitate plants in a trait-mediated tri-trophic cascade. Biol. Lett. 7, 510-513.

Guimarães, P.R., Cogni, R., 2002. Seed cleaning of Cupania vernalis (Sapindaceae) by ants: edge effect in a highland forest in South-East Brazil. J. Trop. Ecol. 18, 303-307.

Guimarães, P.R., Rico-Gray, V., Oliveira, P.S., Izzo, T.J., dos Reis, S.F., Thompson, J.N., 2007. Interaction intimacy affects structure and coevolutionary dynamics in mutualistic networks. Curr. Biol. 17, 1797-1803.

Guimarães, P.R., Galetti, M., Jordano, P., 2008. Seed dispersal anachronisms: rethinking the fruits extinct megafauna ate. PLoS One 3, e1745.

Guimarães, P.R., Jordano, P., Thompson, J.N., 2011. Evolution and coevolution in mutualistic networks. Ecol. Lett. 14, 877-885.

Guimerà, R., Amaral, L.A.N., 2005. Cartography of complex networks: modules and universal roles. J. Stat. Mech. 2005 (2), P02001. http://dx.doi.org/10.1088/1742-5468/ 2005/02/P02001.

Hadley, A.S., Betts, M.G., 2012. The effects of landscape fragmentation on pollination dynamics: absence of evidence not evidence of absence. Biol. Rev. 87, 526-544.

Hagen, M., Kraemer, M., 2010. Agricultural surroundings support flower-visitor networks in an Afrotropical rain forest. Biol. Conserv. 143, 1654-1663.

Hagen, M., Wikelski, M., Kissling, W.D., 2011. Space use of bumblebees (Bombus spp.) revealed by radio-tracking. PLoS One 6, e19997.

Halloy, S.R.P., 1998. A theoretical framework for abundance distributions in complex systems. Complex. Int. 6, 1-12. 
Halloy, S.R.P., Barratt, B.I.P., 2007. Patterns of abundance and morphology as indicators of ecosystem status: a meta-analysis. Ecol. Complex. 4, 128-147.

Hance, T., Baaren, J., van Vernon, P., Boivin, G., 2007. Impact of extreme temperatures on parasitoids in a climate change perspective. Annu. Rev. Entomol. 52, 107-126.

Hanski, I., 1998. Metapopulation dynamics. Nature 396, 41-49.

Hanski, I., 1999. Metapopulation Ecology. Oxford University Press Inc., New York.

Hanski, I., 2005. Landscape fragmentation, biodiversity loss and the societal response- the longterm consequences of our use of natural resources may be surprising and unpleasant. EMBO Rep. 6, 388-392.

Hanski, I., Gilpin, M.E., 1997. Metapopulation Biology, Ecology, Genetics and Evolution. Academic Press, San Diego, CA.

Hanski, I., Ovaskainen, O., 2000. The metapopulation capacity of a fragmented landscape. Nature 404, 755-758.

Hanski, I., Simberloff, D., 1997. The metapopulation approach, its history, conceptual domain and application to conservation. In: Hanski, I.A., Gilpin, M.E. (Eds.), Metapopulation Biology, Ecology, Genetics and Evolution. Academic Press, San Diego, CA, pp. 5-26.

Hansson, L., 1991. Dispersal and connectivity in metapopulations. Biol.J. Linn. Soc. 42, 89-103.

Hansson, L., 1994. Vertebrate distributions relative to clear-cut edges in a boreal forest landscape. Landsc. Ecol. 9, 105-115.

Harder, L.D., 1985. Morphology as a predictor of flower choice by bumble bees. Ecology 66, 198-210.

Hardt, R.A., Forman, R.T.T., 1989. Boundary form effects on woody colonization of reclaimed surface mines. Ecology 70, 1252-1260.

Harris, F.L., Johnson, S.D., 2004. The consequences of habitat fragmentation for plantpollinator mutualisms. Int. J. Trop. Insect Sci. 24, 29-43.

Harris, R.M.L., Milner, A.M., Armitage, P.D., Ledger, M.E., 2007. Replicability of physicochemistry and macroinvertebrate assemblages in stream mesocosms, implications for experimental research. Freshw. Biol. 52, 2434-2443.

Haskell, J.P., Ritchie, M.E., Olff, H., 2002. Fractal geometry predicts varying body size scaling relationships for mammal and bird home ranges. Nature 418, 527-530.

Hastings, A., Powell, T., 1991. Chaos in a three-species food chain. Ecology 72, 896-903.

Hegland, S.J., Totland, Ø., 2008. Is the magnitude of pollen limitation in a plant community affected by pollinator visitation and plant species specialisation levels? Oikos 117, 883-891.

Hegland, S.J., Nielsen, A., Lázaro, A., Bjerknes, A.-L., Totland, Ø., 2009. How does climate warming affect plant-pollinator interactions? Ecol. Lett. 12, 184-195.

Heleno, R.H., Ceia, R.S., Ramos, J.A., Memmott, J., 2009. Effects of alien plants on insect abundance and biomass: a food-web approach. Conserv. Biol. 23, 410-419.

Henle, K., Davies, K.F., Kleyer, M., Margules, C., Settele, J., 2004. Predictors of species sensitivity to fragmentation. Biodivers. Conserv. 13, 207-251.

Henri, D.C., van Veen, F.J.F., 2011. Body size, life history and the structure of hostparasitoid networks. Adv. Ecol. Res. 45, 135-180.

Herrera, C.M., 1988. Variation in mutualisms, the spatiotemporal mosaic of a pollinator assemblage. Biol. J. Linn. Soc. 35, 95-125.

Herrera, C.M., 2009. Multiplicity in Unity. Plant Subindividual Variation and Interactions with Animals. University of Chicago Press, Chicago.

Hildrew, A.G., 2009. Sustained research on stream communities: a model system and the comparative approach. Adv. Ecol. Res. 41, 175-312.

Hildrew, A.G., Woodward, G., Winterbottom, J.H., Orton, S., 2004. Strong densitydependence in a predatory insect: larger scale experiments in a stream. J. Anim. Ecol. $73,448-458$. 
Hill, J.K., Gray, M.A., Khen, C.V., Benedick, S., Tawatao, N., Hamer, K.C., 2011. Ecological impacts of tropical forest fragmentation: how consistent are patterns in species richness and nestedness? Philos. Trans. R. Soc. B 366, 3265-3276.

Hilty, J.A., Lidicker Jr., W.Z., Merenlender, A.M., 2006. Corridor Ecology: The Science and Practice of Linking Landscapes for Biodiversity Conservation. Island Press, Washington, DC.

Hirst, J.A., Attrill, M.J., 2008. Small is beautiful: an inverted view of habitat fragmentation in seagrass beds. Estuar. Coast. Shelf Sci. 78, 811-818.

Hladyz, S., Gessner, M.O., Giller, P.S., Pozo, J., Woodward, G., 2009. Resource quality and stochiometric constraints in a stream food web. Freshw. Biol. 54, 957-970.

Hladyz, S., Åbjörnsson, K., Cariss, H., Chauvet, E., Dobson, M., Elosegi, A., Ferreira, V., Fleituch, T., Gessner, M.O., Giller, P.S., Graça, M.A.S., Gulis, V., et al., 2011a. Stream ecosystem functioning in an agricultural landscape: the importance of terrestrial-aquatic linkages. Adv. Ecol. Res. 44, 211-276.

Hladyz, S., Åbjörnsson, K., Giller, P.S., Woodward, G., 2011b. Impacts of an aggressive riparian invader on community structure and ecosystem functioning in stream food webs. J. Appl. Ecol. 48, 443-452.

Hobbs, R.J., Yates, C.J., 2003. Impacts of ecosystem fragmentation on plant populations: generalising the idiosyncratic. Aust. J. Bot. 51, 471-488.

Hobbs, R.J., Richardson, D.M., Davis, G.W., 1995. Mediterranean-type ecosystems, opportunities and constraints for studying the function of biodiversity. In: Davis, G.W., Richardson, D.M. (Eds.), Mediterranean-Type Ecosystem-The Function of Biodiversity. Springer-Verlag, Berlin, pp. 1-32.

Hoekman, D., Dreyer, J., Jackson, R.D., Townsend, P.A., Gratton, C., 2011. Lake to land subsidies: experimental addition of aquatic insects increases terrestrial arthropod densities. Ecology 92, 2063-2072.

Hoekstra, H.E., Fagan, W.F., 1998. Body size, dispersal ability and compositional disharmony, the carnivore-dominated fauna of the Kuril Islands. Divers. Distrib. 4, 135-149.

Holt, R.D., 1996. Food webs in space: an island biogeographic perspective. In: Polis, G.A., Winemiller, K.O. (Eds.), Food Webs, Integration of Patterns and Dynamics. Chapman and Hall, London, pp. 313-323.

Holt, R.D., 1997. From metapopulation dynamics to community structure some consequences of spatial heterogeneity. In: Hanski, I.A., Gilpin, M.E. (Eds.), Metapopulation Biology. Academic Press, San Diego, CA, pp. 149-164.

Holt, R.D., 2002. Food webs in space: on the interplay of dynamic instability and spatial processes. Ecol. Res. 17, 261-273.

Holyoak, M., 2000. Habitat subdivision causes changes in food web structure. Ecol. Lett. 3, 509-515.

Holzschuh, A., Steffan-Dewenter, I., Tscharntke, T., 2010. How do landscape composition and configuration, organic farming and fallow strips affect the diversity of bees, wasps and their parasitoids? J. Anim. Ecol. 79, 491-500.

Horvitz, C.C., Schemske, D.W., 1990. Spatiotemporal variation in insect mutualists of a neotropical herb. Ecology 71, 1085-1097.

Hovel, K.A., Lipcius, R.N., 2001. Habitat fragmentation in a seagrass landscape: patch size and complexity control blue crab survival. Ecology 82, 1814-1829.

Hubbell, S.P., 2001. The Unified Neutral Theory of Biodiversity and Biogeography. Princeton University Press, Princeton, MA.

Hughes, T.P., 1994. Catastrophes, phase-shifts, and large-scale degradation of a Caribbean coral-reef. Science 265, 1547-1551.

Ings, T.C., Montoya, J.M., Bascompte, J., Blüthgen, N., Brown, L., Dormann, C.F., Edwards, F., Figueroa, D., Jacob, U., Jones, J.I., Lauridsen, R.B., Ledger, M.E., et al., 2009. Ecological networks—-beyond food webs. J. Anim. Ecol. 78, 253-269. 
Jacob, U., Thierry, A., Brose, U., Arntz, W.E., Berg, S., Brey, T., Fetzer, I., Jonsson, T., Mintenbeck, K., Mollmann, C., Petchey, O., Riede, J.O., et al., 2011. The role of body size in complex food webs: a cold case. Adv. Ecol. Res. 45, 181-223.

Jacobsen, D., 2008. Low oxygen pressure as a driving factor for the altitudinal decline in taxon richness of stream macroinvertebrates. Oecologia 154, 795-807.

Jacobsen, D., Milner, A.M., Brown, L.E., Dangles, O., 2012. Biodiversity under threat in glacier-fed river systems. Nat. Clim. Change. 2, 361-364.

Janzen, D.H., 1971. Euglossine bees as long-distance pollinators of tropical plants. Science 171, 203-205.

Jennersten, O., 1988. Pollination in Dianthus deltoides (Caryophyllaceae): effects of habitat fragmentation on visitation and seed set. Conserv. Biol. 2, 359-366.

Jennings, S., Warr, K.J., 2003. Smaller predator-prey body size ratios in longer food chains. Proc. R. Soc. B 270, 1413-1417.

Jetz, W., Carbone, C., Fulford, J., Brown, J.H., 2004. The scaling of animal space use. Science 306, 266-268.

Jetz, W., Wilcove, D.S., Dobson, A.P., 2007. Projected impacts of climate and land-use change on the global diversity of birds. PLoS Biol. 5, 1211-1219.

Jonsson, T., Ebenman, B., 1998. Effects of predator-prey body size ratios on the stability of food chains. J. Theor. Biol. 193, 407-417.

Jonsson, T., Cohen, J.E., Carpenter, S.R., 2005. Food webs, body size, and species abundance in ecological community description. Adv. Ecol. Res. 36, 1-84.

Jordano, P., 1987. Patterns of mutualistic interactions in pollination and seed dispersal — connectance, dependence asymmetry and coevolution. Am. Nat. 129, 657-677.

Jordano, P., 1995. Angiosperm fleshy fruits and seed dispersers-a comparative analysis of adaptation and constraints in plant-animal interactions. Am. Nat. 145, 163-191.

Jordano, P., 2000. Fruits and frugivory. In: Fenner, M. (Ed.), Seeds: The Ecology of Regeneration in Natural Plant Communities. Commonwealth Agricultural Bureau International, Wallingford, UK, pp. 125-166.

Jordano, P., Schupp, E.W., 2000. Seed disperser effectiveness, the quantity component and patterns of seed rain for Prunus mahaleb. Ecol. Monogr. 70, 591-615.

Jordano, P., Bascompte, J., Olesen, J.M., 2003. Invariant properties in coevolutionary networks of plant-animal interactions. Ecol. Lett. 6, 69-81.

Jules, E.S., Rathcke, B.J., 1999. Mechanisms of reduced Trillium recruitment along edges of old-growth forest fragments. Conserv. Biol. 13, 784-793.

Kaartinen, R., Roslin, T., 2011. Shrinking by numbers: landscape context affects the species composition but not the quantitative structure of local food webs. J. Anim. Ecol. 80, 622-631.

Kaiser, C.N., Hansen, O.M., Müller, C.B., 2008. Habitat structure affects reproductive success of the rare endemic tree syzygium mamillatum (Myrtaceae) in restored and unrestored sites in Mauritius. Biotropica 40, 86-94.

Kaiser-Bunbury, C.N., Muff, S., Memmott, J., Müller, C.B., Caflisch, A., 2010. The robustness of pollination networks to the loss of species and interactions, a quantitative approach incorporating pollinator behaviour. Ecol. Lett. 13, 442-452.

Kaiser-Bunbury, C.N., Traveset, A., Hansen, D.M., 2010. Conservation and restoration of plant-animal mutualisms on oceanic islands. Pl. Ecol. Evol. Syst. 12, 131-143.

Kaiser-Bunbury, C.N., Valentin, T., Mougal, J., Matatiken, D., Ghazoul, J., 2011. The tolerance of island pollination networks to alien plants. J. Ecol. 99, 202-213.

Kandori, I., 2002. Diverse visitors with various pollinator importance and temporal change in the important pollinators of Geranium thunbergii (Geraniaceae). Ecol. Res. 17, 283-294.

Kapos, V., Wandelli, E., Camargo, J.L., Ganade, G., 1997. Edge-related changes in environmental and plant responses due to forest fragmentation in Central Amazonia. In: 
Laurance, W.F., Bierregaard, R.O. (Eds.), Tropical Forest Remnants. Chicago University Press, Chicago, IL, pp. 33-44.

Kato, E., Hiura, T., 1999. Fruit set in Styrax obassia (Styracaceae): the effect of light availability, display size and local floral density. Am. J. Bot. 86, 495-501.

Kearns, C.A., 2001. North American, dipteran pollinators: assessing their value and conservation status. Conserv. Ecol. 5, 5.

Kearns, C.A., Inouye, D.W., Waser, N.M., 1998. Endangered mutualisms: the conservation of plant-pollinator interactions. Annu. Rev. Ecol. Syst. 29, 83-112.

Kingsolver, J.G., Hoekstra, H.E., Hoekstra, J.M., Berrigan, D., Vignieri, S.N., Hill, C.E., Hoang, A., Gibert, P., Beerli, P., 2001. The strength of phenotypic selection in natural populations. Am. Nat. 157, 245-261.

Kissling, W.D., Rahbek, C., Böhning-Gaese, K., 2007. Food plant diversity as broad-scale determinant of avian frugivore richness. Proc. R. Soc. B 274, 799-808.

Kissling, W.D., Field, R., Böhning-Gaese, K., 2008. Spatial patterns of woody plant and bird diversity: functional relationships or environmental effects? Glob. Ecol. Biogeogr. 17, 327-339.

Kissling, W.D., Böhning-Gaese, K., Jetz, W., 2009. The global distribution of frugivory in birds. Glob. Ecol. Biogeogr. 18, 150-162.

Kissling, W.D., Field, R., Korntheuer, H., Heyder, U., Böhning-Gaese, K., 2010. Woody plants and the prediction of climate-change impacts on bird diversity. Philos. Trans. R. Soc. B 365, 2035-2045.

Kissling, W.D., Dormann, C.F., Groeneveld, J., Hickler, T., Kühn, I., McInerny, G.J., Montoya, J.M., Römermann, C., Schiffers, K., Schurr, F.M., Singer, A., Svenning, J.-C., et al., 2012a. Towards novel approaches to modelling biotic interactions in multispecies assemblages at large spatial extents. J. Biogeogr. http://dx.doi.org/ 10.1111/j.1365-2699.2011.02663.x.

Kissling, W.D., Sekercioglu, C.H., Jetz, W., 2012b. Bird dietary guild richness across latitudes, environments and biogeographic regions. Glob. Ecol. Biogeogr. 21, 328-340.

Kitching, R.L., 2000. Food Webs and Container Habitats: The Natural History and Ecology of Phytotelmata. Cambridge University Press, Cambridge, UK p. 431.

Kitching, R.L., 2001. Food webs in phytotelmata: "bottom-up" and "top-down" explanations for community structure. Annu. Rev. Entomol. 46, 729-760.

Kivinen, S., Luoto, M., Kuussaari, M., Helenius, J., 2006. Multi-species richness of boreal agricultural landscapes: effects of climate, biotope, soil and geographical location. J. Biogeogr. 33, 862-875.

Klausmeier, C.A., 2001. Habitat destruction and extinction in competitive and mutualistic metacommunities. Ecol. Lett. 4, 57-63.

Klein, A.-M., Steffan-Dewenter, I., Tscharntke, T., 2003. Pollination of Coffea canephora in relation to local and regional agroforestry management. J. Appl. Ecol. 40, 837-845.

Kneitel, J.M., Miller, T.E., 2002. Resource and top-predator regulation in the pitcher plant (Sarracenia purpurea) inquiline community. Ecology 83, 680-688.

Koh, L.P., 2007. Impacts of land use change on South-east Asian forest butterflies: a review. J. Appl. Ecol. 44, 703-713.

Kolb, A., Diekmann, M., 2005. Effects of life-history traits on responses of plant species to forest fragmentation. Conserv. Biol. 19, 929-938.

Kondoh, M., Kato, S., Sakato, Y., 2010. Food webs are built up with nested subwebs. Ecology 91, 3123-3130.

Körner, C., 1993. Scaling from species to vegetation, the usefulness of functional groups. In: Schulze, E.D., Mooney, H.A. (Eds.), Biodiversity and Ecosystem Function. SpringerVerlag, Berlin, pp. 117-140. 
Kraemer, M., Schmitt, U., 1999. Possible pollination by hummingbirds in Anthurium sanguineum Engl. (Araceae). Plant Syst. Evol. 217, 333-335.

Krauss, J., Alfert, T., Steffan-Dewenter, I., 2009. Habitat area but not habitat age determines wild bee richness in limestone quarries. J. Appl. Ecol. 46, 194-202.

Krauss, J., Bommarco, R., Guardiola, M., Heikkinen, R.K., Helm, A., Kuussaari, M., Lindborg, R., Öckinger, E., Pärtel, M., Pino, J., Pöyry, J., Raatikainen, K.M., et al., 2010. Habitat fragmentation causes immediate and time-delayed biodiversity loss at different trophic levels. Ecol. Lett. 13, 597-605.

Kremen, C., Hall, G., 2005. Managing ecosystem services: what do we need to know about their ecology? Ecol. Lett. 8, 468-479.

Krishna, A., Guimarães Jr., P.R., Jordano, P., Bascompte, J., 2008. A neutral-niche theory of nestedness in mutualistic networks. Oikos 117, 1609-1618.

Kruess, A., 2003. Effects of landscape structure and habitat type on a plant-herbivoreparasitoid community. Ecography 26, 283-290.

Kruess, A., Tscharntke, T., 1994. Habitat fragmentation, species loss and biological control. Science 264, 1581-1584.

Kruess, A., Tscharntke, T., 2000. Species richness and parasitism in a fragmented landscape: experiments and field studies with insects on Vicia sepium. Oecologia 122, $129-137$.

Kundzewicz, Z.W., Mata, L.J., Arnell, N.W., Döll, P., Jimenez, B., Miller, K., Oki, T., Sem, Z., Shiklomanov, I., 2008. The implications of projected climate change for freshwater resources and their management. Hydrol. Sci. J. 53, 3-10.

Kurihara, Y., 1959. Synecological analyses of the biotic community in microcosm. IV. Studies on the relations of Diptera larvae to $\mathrm{pH}$ in bamboo containers. Sci. Rep. Tohuku Univ. Ser. IV Biol. 25, 165-171.

Kuussaari, M., Nieminen, M., Hanski, I., 1996. An experimental study of migration in the Glanville Fritillary Butterfly Melitaea cinxia. J. Anim. Ecol. 65, 791-801.

Lafferty, K.D., Allesina, S., Arim, M., Briggs, C.J., De Leo, G., Dobson, A.P., Dunne, J.A., Johnson, P.T.J., Kuris, A.M., Marcogliese, D.J., Martinez, N.D., Memmott, J., et al., 2008. Parasites in food webs: the ultimate missing links. Ecol. Lett. 11, 533-546.

Lake, P.S., 2003. Ecological effects of perturbation by drought in flowing water. Freshw. Biol. 48, 1161-1172.

Laliberté, E., Tylianakis, J.M., 2010. Deforestation homogenizes tropical parasitoid-host networks. Ecology 91, 1740-1747.

Lamont, B.B., Barker, M.J., 1988. Seed bank dynamics of a serotinous, non-sprouting Banksia species. Aust. J. Bot. 36, 193-203.

Lamont, B.B., Klinkhamer, P.G.L., Witkowski, E.T.F., 1993. Population fragmentation may reduce fertility to zero in Banksia goodii-a demonstration of the Allee effect. Oecologia 94, 446-450.

Lancaster, J., 1996. Scaling the effects of predation and disturbance in a patchy environment. Oecologia 107, 321-331.

Lancaster, J., Bradley, D.C., Hogan, A., Waldron, S., 2005. Intraguild omnivory in predatory stream insects. J. Anim. Ecol. 74, 619-629.

Lande, R., 1988. Genetics and demography in biological conservation. Science 241, 1455-1460.

Lander, T.A., Bebber, D.P., Choy, C.T., Harris, S.A., Boshier, D.H., 2011. The Circe principle explains how resource-rich land can waylay pollinators in fragmented landscapes. Curr. Biol. 9, 1302-1307.

Laube, I., Breitbach, N., Böhning-Gaese, K., 2008. Avian diversity in a Kenyan agroecosystem: effects of habitat structure and proximity to forest. J. Ornithol. 149, 181-191.

Laurance, S.G.W., 2004. Responses of understory rain forest birds to road edges in Central Amazonia. Ecol. Appl. 14, 1344-1357. 
Laurance, W.F., Laurance, S.G.W., Ferreira, L.V., Rankin-de Merona, J.M., Gascon, C., Lovejoy, T.E., 1997. Biomass collapse in Amazonian forest fragments. Science 278, $1117-1118$.

Laurance, W.F., Ferreira, L.V., Rankin-De Merona,J.M., Laurance, S.G., 1998. Rain forest fragmentation and the dynamics of Amazonian tree communities. Ecology 79, 2032-2040.

Laurance, W.F., Nascimento, H.E.M., Laurance, S.G., Andrade, A.C., Fearnside, P.M., Ribeiro, J.E.L., Capretz, R.L., 2006a. Rain forest fragmentation and the proliferation of successional trees. Ecology 87, 469-482.

Laurance, W.F., Nascimento, H.E.M., Laurance, S.G., Andrade, A., Ribeiro, J.E.L.S., Giraldo, J.P., Lovejoy, T.E., Condit, R., Chave, J., Harms, K.E., D’Angelo, S., 2006b. Rapid decay of tree-community composition in Amazonian forest fragments. Proc. Natl. Acad. Sci. USA 103, 19010-19014.

Laurance, W.F., Nascimento, H.E.M., Laurance, S.G., Andrade, A., Ewers, R.M., Harms, K.E., Luizão, R.C.C., Ribeiro, J.E., 2007. Habitat fragmentation, variable edge effects and the landscape-divergence hypothesis. PLoS One 2, e1017.

Laurance, W.F., Camargo, J.L.C., Luizão, R.C.C., Laurance, S.G., Pimm, S.L., Bruna, E.M., Stouffer, P.C., Williamson, G.B., Benítez-Malvido, J., Vasconcelos, H.L., van Houtan, K.S., Zartman, C.E., et al., 2011. The fate of Amazonian forest fragments: a 32-year investigation. Biol. Conserv. 144, 56-67.

Lavandier, P., Céréghino, R., 1995. Use and partition of space and resources by two coexisting Rhyacophila species (Trichoptera) in a high mountain stream. Hydrobiologia 300/301, 157-162.

Lavandier, P., Décamps, H., 1983. Un torrent d'altitude dans les Pyrénées, l'Estaragne. In: Bourliere, F., Lamotte, M. (Eds.), Ecosystemes Limniques. Masson, Paris, pp. 81-111.

Layer, K., Riede, J.O., Hildrew, A.G., Woodward, G., 2010. Food web structure and stability in 20 streams across a wide $\mathrm{pH}$ gradient. Adv. Ecol. Res. 42, 265-299.

Layer, K., Hildrew, A.G., Jenkins, G.B., Riede, J., Rossiter, S.J., Townsend, C.R., Woodward, G., 2011. Long-term dynamics of a well-characterised food web: four decades of acidification and recovery in the Broadstone Stream model system. Adv. Ecol. Res. 44, 69-117.

Leck, C.F., 1979. Avian extinctions in an isolated tropical wet-forest preserve, Ecuador. Auk 96, 343-352.

Ledger, M.E., Harris, R.M.L., Armitage, P.D., Milner, A.M., 2008. Disturbance frequency influences patch dynamics in stream benthic algal communities. Oecologia 155, 809-819.

Ledger, M.E., Harris, R.M.L., Armitage, P.D., Milner, A.M., 2009. Realism of model ecosystems: an evaluation of physicochemistry and macroinvertebrate assemblages in artificial streams. Hydrobiologia 617, 91-99.

Ledger, M.E., Edwards, F.K., Brown, L.E., Milner, A.M., Woodward, G., 2011. Impact of simulated drought on ecosystem biomass production: an experimental test in stream mesocosms. Glob. Change Biol. 17, 2288-2297.

Ledger, M.E., Harris, R.M.L., Armitage, P.D., Milner, A.M., 2012. Climate change impacts on community resilience: experimental evidence from a drought disturbance experiment. Adv. Ecol. Res. 46, 211-258.

Lees, A.C., Peres, C.A., 2009. Gap-crossing movements predict species occupancy in Amazonian forest fragments. Oikos 118, 280-290.

Leibold, M.A., Holyoak, M., Mouquet, N., Amarasekare, P., Chase, J.M., Hoopes, M.F., Holt, R.D., Shurin, J.B., Law, R., Tilman, D., Loreau, M., Gonzalez, A., 2004. The metacommunity concept: a framework for multi-scale community ecology. Ecol. Lett. 7, 601-613.

Lenz, J., Fiedler, W., Caprano, T., Friedrichs, W., Gaese, B.H., Wikelski, M., Böhning-Gaese, K., 2011. Seed-dispersal distributions by trumpeter hornbills in fragmented landscapes. Proc. R. Soc. Lond. B 278, 2257-2264. 
Levin, S.A., 1992. The problem of pattern and scale in ecology. Ecology 73, 1943-1967.

Lewinsohn, T.M., Novotny, V., Basset, Y., 2005. Insects on plants: diversity of herbivore assemblages revisited. Annu. Rev. Ecol. Syst. 36, 597-620.

Lewinsohn, T.M., Prado, P.I., Jordano, P., Bascompte, J., Olesen, J.M., 2006. Structure in plant-animal interaction assemblages. Oikos 113, 174-184.

Lidicker, W., 1985. An overview of dispersal in non-volant small mammals. Contrib. Mar. Sci. Suppl. 27, 359-375.

Lidicker, W., 1999. Responses of mammals to habitat edges: an overview. Landsc. Ecol. 14, 333-343.

Lindenmayer, D.B., Luck, G., 2005. Synthesis: thresholds in conservation and management. Biol. Conserv. 124, 351-354.

Lindstedt, S.L., Miller, B.J., Buskirk, S.W., 1986. Home range, time, and body size in mammals. Ecology 67, 413-418.

Lomolino, M.V., 1985. Body size of mammals on islands: the island rule reexamined. Am. Nat. $125,310-316$.

Lopes, A.V., Girão, L.C., Santos, B.A., Peres, C.A., Tabarelli, M., 2009. Long-term erosion of tree reproductive trait diversity in edge-dominated Atlantic forest fragments. Biol. Conserv. 142, 1154-1165.

Lopezaraiza-Mikel, M.E., Hayes, R.B., Whalley, M.R., Memmott, J., 2007. The impact of an alien plant on a native plant pollinator network: an experimental approach. Ecol. Lett. 10, 539-550.

Lord, J.M., 2004. Frugivore gape size and the evolution of fruit size and shape in southern hemisphere floras. Austral Ecol. 29, 430-436.

Luck, G.W., Daily, G.C., 2003. Tropical countryside bird assemblages: richness, composition, and foraging differ by landscape context. Ecol. Appl. 13, 235-247.

MacArthur, R., 1955. Fluctuations of animal populations and a measure of community stability. Ecology 36, 533-536.

MacArthur, R.H., Pianka, E.R., 1966. On optimal use of a patchy environment. Am. Nat. 100, 603-609.

MacArthur, R.H., Wilson, E.O., 1963. An equilibrium theory of insular zoogeography. Evolution 17, 373-387.

MacArthur, R.H., Wilson, E.O., 1967. The Theory of Island Biogeography. Princeton University Press, Princeton, MA.

MacFadyen, S., Gibson, R.H., Symondson, W.O.C., Memmott, J., 2011. Landscape structure influences modularity patterns in farm food webs: consequences for pest control. Ecol. Appl. 21, 516-524.

Macreadie, P.I., Hindell, J.S., Jenkins, G.P., Connolly, R.M., Keough, M.J., 2009. Fish responses to experimental fragmentation of seagrass habitat. Conserv. Biol. 23, 644-652.

Maionchi, D.O., dos Reis, S.F., de Aguiar, M.A.M., 2006. Chaos and pattern formation in a spatial tritrophic food chain. Ecol. Modell. 191, 291-303.

Malard, F., Uehlinger, U., Zah, R., Tockner, K., 2006. Flood-pulse and riverscape dynamics in a braided glacial river. Ecology 87, 704-716.

Marquitti, F.M.D., 2011. Interaction networks between frugivorous bats and plants, geographical variation and niche conservatism. MSc Thesis. Universidade Estadual de Campinas, Brazil. http://www.bibliotecadigital.unicamp.br/document/?code $=000796920$.

Matthies, D., Bräuer, I., Maibom, W., Tscharntke, T., 2004. Population size and the risk of local extinction: empirical evidence from rare plants. Oikos 105, 481-488.

May, R.M., 1972. Will a large complex system be stable? Nature 238, 413-414.

May, R.M., 1973. Stability in randomly fluctuating versus deterministic environments. Am. Nat. 107, 621-650. 
McCann, K.S., Hastings, A., Huxel, G.R., 1998. Weak trophic interactions and the balance of nature. Nature 395, 794-798.

McCann, K.S., Rasmussen, J.R., Umbanhowar, J., 2005a. The dynamics of spatially coupled food webs. Ecol. Lett. 8, 513-523.

McCann, K.S., Rasmussen, J.R., Umbanhowar, J., Humphries, M., 2005b. The role of space, time and variability in food web dynamics. In: de Ruiter, P.C., Wolters, V., Moore, J.C. (Eds.), Dynamic Food Webs, Multispecies Assemblages, Ecosystem Development and Environmental Change. Elsevier and Academic Press, Burlington, MA, pp. 56-70.

McConkey, K., Drake, D., 2006. Flying foxes cease to function as seed dispersers long before they become rare. Ecology 87, 271-276.

McKinney, M.L., Lockwood, J.L., 1999. Biotic homogenization: a few winners replacing many losers in the next mass extinction. Trends Ecol. Evol. 14, 450-453.

McLaughlin, O.B., Jonsson, T., Emmerson, M.C., 2010. Temporal variability in predatorprey relationships of a forest floor food web. Adv. Ecol. Res. 42, 171-264 3.

Meerhoff, M., Mello, F.T-d., Kruk, C., Alonso, C., González-Bergonzoni, I., Pablo Pacheco, J., Lacerot, G., Arim, M., Beklioğlu, M., Brucet, S., Goyenola, G., Iglesias, C., Mazzeo, N., Kosten, S., Jeppesen, E., et al., 2012. Environmental warming in shallow lakes: a review of potential changes in community structure as evidenced from spacefor-time substitution approaches. Adv. Ecol. Res. 46, 259-350.

Melián, C.J., Vilas, C., Baldó, F., González-Ortegón, E., Drake, P., Williams, R.J., 2011. Ecoevolutionary dynamics of individual-based food webs. Adv. Ecol. Res. 45, 225-268.

Melo, F.P.L., Dirzo, R., Tabarelli, M., 2006. Biased seed rain in forest edges: evidence from the Brazilian Atlantic forest. Biol. Conserv. 132, 50-60.

Memmott, J., 1999. The structure of a plant-pollinator food web. Ecol. Lett. 2, 276-280.

Memmott, J., Waser, N.M., Price, M.V., 2004. Tolerance of pollination networks to species extinctions. Proc. R. Soc. Lond. B 271, 2605-2611.

Memmott, J., Craze, P.G., Waser, N.M., Price, M.V., 2007. Global warming and the disruption of plant-pollinator interactions. Ecol. Lett. 10, 710-717.

Menges, E.S., 1991. The application of minimum viable population theory to plants. In: Falk, D.A., Holsinger, K.E. (Eds.), Genetics and Conservation of Rare Plants. Oxford University Press, New York, pp. 45-61.

Metzger, J.P., Martensen, A.C., Dixo, M., Bernacci, L.C., Ribeiro, M.C., Teixeira, A.M.G., Pardini, R., 2009. Time-lag in biological responses to landscape changes in a highly dynamic Atlantic forest region. Biol. Conserv. 142, 1166-1177.

Michener, C.D., 2007. The Bees of the World, second ed. John Hopkins University Press, Baltimore, MD.

Millennium Ecosystem Assessment, 2005. Ecosystems and Human Wellbeing: Synthesis. Island Press, Washington, DC.

Milner, A.M., Brittain, J.E., Castella, E., Petts, G.E., 2001. Trends in macroinvertebrate community structure in glacier-fed rivers in relation to environmental conditions, a synthesis. Freshw. Biol. 46, 1833-1847.

Milner, A.M., Brown, L.E., Hannah, D.M., 2009. Hydroecological response of river systems to shrinking glaciers. Hydrol. Process 23, 62-77.

Milner, A.M., Robertson, A.L., Brown, L.E., Sonderland, S., McDermott, M., Veal, A.J., 2011. Evolution of a stream ecosystem in recently deglaciated terrain. Ecology 92, 1924-1935.

Milton, K., May, M.L., 1976. Body-weight, diet and home range area in primates. Nature 259, 459-462.

Mimura, M., Murray, J.D., 1978. On a diffusive prey-predator model which exhibits patchiness. J. Theor. Biol. 75, 249-262.

Mintenbeck, K., Barrera-Oro, E.R., Brey, T., Jacob, U., Knust, R., Mark, F.C., Moreira, E., Strobel, A., Arntz, W.E., et al., 2012. Impact of Climate Change on Fishes in Complex Antarctic Ecosystems. Adv. Ecol. Res. 46, 351-426. 
Mistro, D.C., Rodrigues, L.A., Varriale, M.C., 2009. The role of spatial refuges in coupled map lattice model for host-parasitoid systems. Bull. Math. Biol. 71, 1934-1953.

Moeller, D.A., 2005. Pollinator community structure and sources of spatial variation in plantpollinator interactions in Clarkia xantiana ssp. xantiana. Oecologia 142, $28-37$.

Molnár, P.K., Derocher, A.E., Klanjscek, T., Lewis, M.A., 2011. Predicting climate change impacts on polar bear litter size. Nat. Commun. 8, 186.

Monaghan, M.T., Spaak, P.T., Robinson, C.T., Ward, J.V., 2002. Population genetic structure of three alpine stream insects, influences of gene flow, demographics, and habitat fragmentation. J. N. Am. Benthol. Soc. 21, 114-131.

Monaghan, M.T., Robinson, C.T., Spaak, P.T., Ward, J.V., 2005. Macroinvertebrate diversity in fragmented alpine streams, implications for freshwater conservation. Aquat. Sci. 67, 454-464.

Montoya, J.M., Emmerson, M.C., Woodward, G., 2005. Perturbations and indirect effects in complex food webs. In: de Ruiter, P.C., Wolters, V., Moore, J.C. (Eds.), Dynamic Food Webs, Multispecies Assemblages, Ecosystem Development, and Environmental Change. Academic Press, Amsterdam, pp. 369-380.

Montoya, D., Zavala, M.A., Rodriguez, M.A., Purves, D.W., 2008. Animal versus wind dispersal and the robustness of tree species to deforestation. Science 320, 1502-1504.

Morales, C.L., Aizen, M.A., 2006. Invasive mutualisms and the structure of plant-pollinator interactions in the temperate forests of north-west Patagonia, Argentina. J. Ecol. 94, 171-180.

Morales, J.M., Carlo, T.S., 2006. The effects of plant distribution and frugivore density on the scale and shape of dispersal kernels. Ecology 87, 1489-1496.

Morales, C.L., Traveset, A., 2009. A meta-analysis of impacts of alien vs. native plants on pollinator visitation and reproductive success of co-flowering native plants. Ecol. Lett. $12,716-728$.

Morales, J.M., Vázquez, D.P., 2008. The effect of space in plant-animal mutualistic networks: insights from a simulation study. Oikos 117, 1362-1370.

Morellato, L.P.C., 2004. Phenology, sex ratio, and spatial distribution among dioecious species of Trichilia (Meliaceae). Plant Biol. 6, 491-497.

Moretti, M., de Bello, F., Roberts, S.P.M., Potts, S.G., 2009. Taxonomical vs. functional responses of bee communities to fire in two contrasting climatic regions. J. Anim. Ecol. 78, 98-108.

Morris, D.W., 1997. Optimally foraging deer mice in prairie mosaics: a test of habitat theory and absence of landscape effects. Oikos 80, 31-42.

Mueller, D.R., Vincent, W.F., Jefries, M.O., 2006. Environmental gradients, fragmented habitats and microbiota of a northern ice shelf cryoecosystem, Ellesmere Island, Canada. Arct. Antarct. Alp. Res. 38, 593-607.

Mulder, C., den Hollander, H.A., Vonk, J.A., Rossberg, A.G., op Akkerhuis, G.A., Yeates, G.W., 2009. Soil resource supply influences faunal size-specific distributions in natural food webs. Naturwissenschaften 96, 813-826.

Mulder, C., Boit, A., Bonkowski, M., de Ruiter, P.C., Mancinelli, G., van der Heijden, M.G.A., van Wijnen, H.J., Vonk, J.A., Rutgers, M., 2011. A belowground perspective on Dutch agroecosystems: how soil organisms interact to support ecosystem services. Adv. Ecol. Res. 44, 277-358.

Mulder, C., Boit, A., Mori, S., Vonk, J.A., Dyer, S.D., Faggiano, L., Geisen, S., González, A.L., Kaspari, M., Lavorel, S., Marquet, P.A., Rossberg, A.G., Sterner, R.W., Voigt, W., Wall, D.H., et al., 2012. Distributional (in)congruence of biodiversity ecosystem functioning. Adv. Ecol. Res. 46, 1-88.

Munday, P.L., 2004. Habitat loss, resource specialization, and extinction on coral reefs. Glob. Change Biol. 10, 1642-1647. 
Murcia, C., 1995. Edge effects in fragmented forests, implications for conservation. Trends Ecol. Evol. 10, 58-62.

Murray, J.D., 1993. Mathematical Biology, second ed. Springer-Verlag, New York.

Myers, N., 1986. Tropical deforestation and a mega-extinction spasm. In: Soulé, M.E. (Ed.), Conservation Biology: The Science of Scarcity and Diversity. Sinauer Associates, Sunderland, MA, pp. 394-409.

Myers, J.H., Krebs, C.J., 1971. Genetic, behavioral, and reproductive attributes of dispersing field voles, Microtus pennsylvanicus and Microtus ochrogaster. Ecol. Monogr. 41, 53-78.

Nakao, H., Mikhailov, A.S., 2010. Turing patterns in network-organized activator-inhibitor systems. Nat. Phys. 6, 544-550.

Nakazawa, T., Ushio, M., Kondoh, M., 2011. Scale dependence of predator-prey mass ratio: determinants and applications. Adv. Ecol. Res. 45, 269-302.

Nams, V.O., 2011. Emergent properties of patch shapes affect edge permeability to animals. PLoS One 6, e21886.

Nee, S., May, R.M., 1992. Dynamics of metapopulations, habitat destruction and competitive coexistence. J. Anim. Ecol. 61, 37-40.

Nee, S., May, R.M., Hassell, M.P., 1997. Two-species metapopulation models. In: Hanski, I.A., Gilpin, M.E. (Eds.), Metapopulation Biology. Academic Press, San Diego, CA, pp. 123-147.

Neutel, A.M., Heesterbeek, J.A.P., de Ruiter, P.C., 2002. Stability in real food webs: weak links in long loops. Science 296, 1120-1123.

Nielsen, A., Bascompte, J., 2007. Ecological networks, nestedness and sampling effort. J. Ecol. 95, 1134-1141.

Nieminen, M., 1996. Risk of population extinction in moths: effect of host plant characteristics. Oikos 76, 475-484.

Nilsson, L.A., Rabakonandrianina, E., Razananaivo, R., Randriamanindry, J.-J., 1992. Long pollinia on eyes: hawk-moth pollination of Cynorkis uniflora Lindley (Orchidaceae) in Madagascar. Bot. J. Linn. Soc. 109, 145-160.

Noss, R.F., 1987. Corridors in real landscapes: a reply to Simberloff and Cox. Conserv. Biol. 1, 159-164.

Nuismer, S.L., Doebeli, M., 2004. Genetic correlations and the coevolutionary dynamics of three-species systems. Evolution 58, 1165-1177.

Nuismer, S.L., Thompson, J.N., 2006. Coevolutionary alternation in antagonistic interactions. Evolution 60, 2207-2217.

Nuismer, S.L., Thompson, J.N., Gomulkiewicz, R., 1999. Gene flow and geographically structured coevolution. Proc. R. Soc. Lond. B Biol. Sci. 266, 605-609.

Nuismer, S.L., Thompson, J.N., Gomulkiewicz, R., 2000. Coevolutionary clines across selection mosaics. Evolution 54, 1102-1115.

Nyhagen, D.F., Kragelund, C., Olesen, J.M., Jones, C.G., 2001. Insular interactions between lizards and flowers, flower visitation by an endemic Mauritian gecko. J. Trop. Ecol. 17, 755-761.

Öckinger, E., Smith, H.G., 2007. Semi-natural grasslands as population sources for pollinating insects in agricultural landscapes. J. Appl. Ecol. 44, 50-59.

Öckinger, E., Schweiger, O., Crist, T.O., Debinski, D.M., Krauss, J., Kuussaari, M., Petersen, J.D., Pöyry, J., Settele, J., Summerville, K.S., Bommarco, R., 2010. Lifehistory traits predict species responses to habitat area and isolation: a cross-continental synthesis. Ecol. Lett. 13, 969-979.

O'Gorman, E.J., Emmerson, M.C., 2009. Perturbations to trophic interactions and the stability of complex food webs. Proc. Natl. Acad. Sci. USA 106, 13393-13398.

O'Gorman, E.J., Emmerson, M.C., 2010. Manipulating interaction strengths and the consequences for trivariate patterns in a marine food web. Adv. Ecol. Res. 42, 301-419. 
O’Gorman, E.J., Jacob, U., Jonsson, T., Emmerson, M.C., 2010. Interaction strength, food web topology and the relative importance of species in food webs. J. Anim. Ecol. 79, 682-692.

O'Gorman, E.J., Yearsley, J.M., Crowe, T.P., Emmerson, M.C., Jacob, U., Petchey, O.L., 2011. Loss of functionally unique species may gradually undermine ecosystems. Proc. R. Soc. B 278, 1886-1893.

Okuyama, T., Holland, J.N., 2008. Network structure properties mediate the stability of mutualistic communities. Ecol. Lett. 11, 208-216.

Olden, J.D., LeRoy Poff, N., Douglas, M.R., Douglas, M.E., Fausch, K.D., 2004. Ecological and evolutionary consequences of biotic homogenization. Trends Ecol. Evol. 19, $18-24$.

Olesen, J.M., 1985. The Macaronesian bird-flower element and its relation to bird and bee opportunists. Bot. J. Linn. Soc. 91, 395-414.

Olesen, J.M., 2000. Exactly how generalised are pollination interactions? In: Totland, Ø., Armbruster, W.S., Fenster, C., Molau, U., Nilsson, L.A., Olesen, J.M., Ollerton, J., Philipp, M., Ågren, J. (Eds.), The Scandinavian Association for Pollination Ecology Honours Knut Fægri. Norwegian Academy of Science and Letters, Oslo, pp. 161-178.

Olesen, J.M., Jain, S., 1994. Fragmented plant populations and their lost interactions. In: Loeschcke, V., Tomiuk, J., Jain, S. (Eds.), Conservation Genetics. Birkhäuser, Basel, pp. 417-426.

Olesen, J.M., Valido, A., 2003. Lizards as pollinators and seed dispersers, an island phenomenon. Trends Ecol. Evol. 18, 177-181.

Olesen, J.M., Eskildsen, L.I., Venkatasamy, S., 2002. Invasion of pollination networks on oceanic islands: importance of invader complexes and endemic super generalists. Divers. Distrib. 8, 181-192.

Olesen, J.M., Bascompte, J., Dupont, Y.L., Jordano, P., 2006. The smallest of all worlds: pollination networks. J. Theor. Biol. 240, 270-276.

Olesen, J.M., Bascompte, J., Dupont, Y.L., Jordano, P., 2007. The modularity of pollination networks. Proc. Natl. Acad. Sci. USA 104, 19891-19896.

Olesen, J.M., Bascompte, J., Elberling, H., Jordano, P., 2008. Temporal dynamics in a pollination network. Ecology 89, 1573-1582.

Olesen, J.M., Bascompte, J., Dupont, Y.L., Elberling, H., Rasmussen, C., Jordano, P., 2010a. Missing and forbidden links in mutualistic networks. Proc. R. Soc. B 278, $725-732$.

Olesen, J.M., Dupont, Y.L., O’Gorman, E., Ings, T.C., Layer, K., Melián, C.J., Trøjelsgaard, K., Pichler, D.E., Rasmussen, C., Woodward, G., 2010b. From Broadstone to Zackenberg: space, time and hierarchies in ecological networks. Adv. Ecol. Res. 42, 1-69.

Olesen, J.M., Dupont, Y.L., Hagen, M., Rasmussen, C., Trøjelsgaard, K., 2012. Structure and dynamics of pollination networks: the past, present and future. In: Patiny, S. (Ed.), Evolution of Plant-Pollinator Relationships. Cambridge University Press, Cambridge, pp. 374-391.

Oliveira, P.S., Galetti, M., Pedroni, F., Morellato, L.P.C., 1995. Seed cleaning by Mycocepurus goeldii ants (Attini) facilitates germination in Hymenaea courbaril (Caesalpiniaceae). Biotropica 27, 518-522.

Oliveira, M.A., Santos, A.M.M., Tabarelli, M., 2008. Profound impoverishment of the large-tree stand in a hyper-fragmented landscape of the Atlantic forest. For. Ecol. Manage. 256, 1910-1917.

Oliver, T.A., Palumbi, S.R., 2009. Distributions of stress-resistant coral symbionts match environmental patterns at local but not regional scales. Mar. Ecol. Prog. Ser. 378, 93-103. 
Ollerton, J., Johnson, S.D., Cranmer, L., Kellie, S., 2003. The pollination ecology of an assemblage of grassland asclepiads in South Africa. Ann. Bot. 92, 807-834.

Ollerton, J., Winfree, R., Tarrant, S., 2011. How many flowering plants are pollinated by animals? Oikos 120, 321-326.

Orth, R.J., Carruthers, T.J.B., Dennison, W.C., Duarte, C.M., Fourqurean, J.W., Heck Jr., K.L., Hughes, A.R., Kendrick, G.A., Kenworthy, W.J., Olyarnik, S., Short, F.T., Waycott, M., et al., 2006. A global crisis for seagrass ecosystems. Bioscience 56, 987-996.

Pacheco, R., Silva, R.R., Morini, M.S., Brandão, C.R.F., 2009. A comparison of the leaf-litter ant fauna in a secondary Atlantic forest with an adjacent pine plantation in southeastern Brazil. Neotrop. Entomol. 38, 55-65.

Palmer, T.M., Stanton, M.L., Young, T.P., Goheen, J.R., Pringle, R.M., Karban, R., 2008. Breakdown of an ant-plant mutualism follows the loss of large herbivores from an African savanna. Science 319, 192-195.

Parc National des Pyrénées, 2005. Document d'Objectifs de la Zone Spéciale de Conservation: Estaubé, Gavarnie, Troumouse, Barroude. Parc National des Pyrénées, Tarbes, France.

Pardini, R., Faria, D., Accacio, G.M., Laps, R.R., Mariano-Neto, E., Paciencia, M.L.B., Dixo, M., Baumgarten, J., 2009. The challenge of maintaining Atlantic forest biodiversity: a multi-taxa conservation assessment of specialist and generalist species in an agroforestry mosaic in southern Bahia. Biol. Conserv. 142, 1178-1190.

Pascual-Hortal, L., Saura, S., 2006. Comparison and development of new graph-based landscape connectivity indices: towards the priorization of habitat patches and corridors for conservation. Landsc. Ecol. 21, 959-967.

Pasquet, R.M.S., Peltier, A., Hufford, M.B., Oudin, E., Saulnier, J., Paul, L., Knudsen, J.T., Herren, H.R., Gepts, P., 2008. Long-distance pollen flow assessment through evaluation of pollinator foraging range suggests transgene escape distances. Proc. Natl. Acad. Sci. USA $105,13456-13461$.

Pauw, A., 2007. Collapse of a pollination web in small conservation areas. Ecology 88, 1759-1769.

Pérez-Barrales, R., Arroyo, J., Armbruster, W.S., 2007. Differences in pollinator faunas may generate geographic differences in floral morphology and integration in Narcissus papyraceus (Amaryllidaceae). Oikos 116, 1904-1918.

Perfecto, I., Vandermeer, J., 2008. Biodiversity conservation in tropical ecosystems: a new paradigm. Ann. N. Y. Acad. Sci. 1134, 173-200.

Perfectti, F., Gómez, J.M., Bosch, J., 2009. The functional consequences of diversity in plant-pollinator interactions. Oikos 118, 1430-1440.

Petanidou, T., Kallimanis, A.S., Tzanopoulos, J., Sgardelis, S.P., Pantis, J.D., 2008. Longterm observation of a pollination network, fluctuation in species and interactions, relative invariance of network structure and implications for estimates of specialization. Ecol. Lett. 11, 564-575.

Petchey, O.L., Gaston, K.J., 2006. Functional diversity: back to basics and looking forward. Ecol. Lett. 9, 741-758.

Petchey, O.L., Beckerman, A., Riede, J., Warren, P., 2008. Size, foraging, and food web structure. Proc. Natl. Acad. Sci. USA 105, 4191-4196.

Petchey, O.L., Brose, U., Rall, B.C., 2010. Predicting the effects of temperature on food web connectance. Philos. Trans. R. Soc. B 365, 2081-2091.

Petersen, I., Winterbottom, J.H., Orton, S., Friberg, N., Hildrew, A.G., 1999. Emergence and lateral dispersal of adult stoneflies and caddis flies from Broadstone Stream. Freshw. Biol. 42, 401-416.

Pimm, S.L., 1984. The complexity and stability of ecosystems. Nature 307, 321-326. 
Pimm, S.L., Lawton, J.H., 1980. Are food webs divided into compartments? J. Anim. Ecol. 49, 879-898.

Pizo, M.A., Oliveira, P.S., 2000. The use of fruits and seeds by ants in the Atlantic forest of southeast Brazil. Biotropica 32, 851-861.

Pizo, M.A., Passos, L., Oliveira, P.S., 2005. Ants as seed dispersers of fleshy diaspores in Brazilian Atlantic forests. In: Forget, P.-M., Lambert, J.E., Hulme, P.E., Wall, S.B.V. (Eds.), Seed Fate. CAB int. Walling ford, UK, pp. 315-330.

Poff, N.L., Zimmerman, J.K.H., 2010. Ecological responses to altered flow regimes: a literature review to inform the science and management of environmental flows. Freshw. Biol. 55, 194-205.

Pollard, E., Yates, T.J., 1993. Monitoring Butterflies for Ecology and Conservation. Chapman and Hall, London.

Prakash, S., de Roos, A.M., 2004. Habitat destruction in mutualistic metacommunities. Theor. Popul. Biol. 65, 153-163.

Pratchett, M.S., Munday, P.L., Wilson, S.K., Graham, N.A.J., Cinner, J.E., Bellwood, D.R., Jones, G.P., Polunin, N.V.C., McClanahan, T.R., 2008. Effects of climate-induced coral bleaching on coral-reef fishes-ecological and economic consequences. Oceanogr. Mar. Biol. 46, 251-296.

Prevedello, J., Vieira, M., 2011. Does the type of matrix matter? A quantitative review of the evidence. Biodivers. Conserv. 19, 1205-1223.

Proctor, M., Yeo, P., Lack, A., 1996. The Natural History of Pollination. Harper Collins, London.

Prugh, L.R., Hodges, K.E., Sinclair, A.R.E., Brashares, J.S., 2008. Effect of habitat area and isolation on fragmented animal populations. Proc. Natl. Acad. Sci. USA 105, 20770-20775.

Pulliam, H.R., 1988. Sources, sinks and population regulation. Am. Nat. 132, 652-661.

Purdy, K.J., Hurd, P.J., Moya-Laraño, J., Trimmer, M., Woodward, G., 2010. Systems biology for ecology: from molecules to ecosystems. Adv. Ecol. Res. 43, 87-149.

Pütz, S., Groeneveld, J., Alves, L.F., Metzger, J.P., Huth, A., 2011. Fragmentation drives tropical forest fragments to early successional states: a modelling study for Brazilian Atlantic forests. Ecol. Modell. 222, 1986-1997.

Raine, N.E., Chittka, L., 2005. Comparison of flower constancy and foraging performance in three bumblebee species (Hymenoptera: Apidae: Bombus). Entomol. Gen. 28, 81-89.

Ramos, F.N., Santos, F.A.M., 2005. Phenology of Psychotria tenuinervis (Rubiaceae) in Atlantic forest fragments: fragment and habitat scales. Can. J. Bot. 83, 1305-1316.

Rand, T.A., Tscharntke, T., 2007. Contrasting effects of natural habitat loss on generalist and specialist aphid natural enemies. Oikos 116, 1353-1362.

Rasmussen, E., 1977. The wasting disease of eelgrass Zostera marina and its effects on environmental factors and fauna. In: McRoy, C.P., Helfferich, C. (Eds.), Seagrass Ecosystems: A Scientific Perspective. Dekker, New York, pp. 1-52.

Rasmussen, I.R., Brødsgaard, B., 1992. Gene flow inferred from seed dispersal and pollinator behavior compared to DNA analysis of restriction site variation in a patchy population of Lotus corniculatus. Oecologia 89, 277-283.

Rathcke, B.J., Jules, E.S., 1993. Habitat fragmentation and plant-pollinator interactions. Curr. Sci. 65, 273-277.

Raymundo, L.J., Maypa, A.P., Gomez, E.D., Cadiz, P., 2007. Can dynamite-blasted reefs recover? A novel, low-tech approach to stimulating natural recovery in fish and coral populations. Mar. Pollut. Bull. 54, 1009-1019.

Reed, B.J., Hovel, K.A., 2006. Seagrass habitat disturbance: how loss and fragmentation of eelgrass (Zostera marina) influences epifaunal community of San Diego Bay, California, USA. Mar. Ecol. Prog. Ser. 326, 115-131. 
Renjifo, L.M., 1999. Composition changes in a subandean avifauna after long-term forest fragmentation. Conserv. Biol. 13, 1124-1139.

Renner, S.S., 1998. Effects of habitat fragmentation on plant-pollinator interactions in the tropics. In: Newbery, D.M., Prins, H.H.T., Brown, N.D. (Eds.), Dynamics of Tropical Communities. Blackwell, London, pp. 339-360.

Restrepo, C., Gómez, N., 1998. Responses of understory birds to anthropogenic edges in a neotropical montane forest. Ecol. Appl. 8, 170-183.

Restrepo, C., Vargas, A., 1999. Seeds and seedlings of two neotropical montane understory shrubs respond differently to anthropogenic edges and treefall gaps. Oecologia 119, 419-426.

Restrepo, C., Gómez, N., Heredia, S., 1999. Anthropogenic edges, treefall gaps and fruitfrugivore interactions in a neotropical montane forest. Ecology 80, 668-685.

Reuman, D.C., Mulder, C., Banašek-Richter, C., Blandenier, M.-F.C., Breure, A.M., Hollander, H.d., Kneitel, J.M., Raffaelli, D., Woodward, G., Cohen, J.E., 2009. Allometry of body size and abundance in 166 food webs. Adv. Ecol. Res. 41, 1-44.

Rezende, E., Lavabre, J.E., Guimarães, P.R., Jordano, P., Bascompte, J., 2007. Nonrandom coextinctions in phylogenetically structured mutualistic networks. Nature 448, 925-928.

Ribeiro, M.C., Metzger, J.P., Martensen, A.C., Ponzoni, F.J., Hirota, M.M., 2009. The Brazilian Atlantic Forest: how much is left and how is the remaining forest distributed? Implications for conservation. Biol. Conserv. 142, 1141-1153.

Ricketts, T., Williams, N.M., Mayfield, M.M., 2006. Connectivity and ecosystem services: crop pollination in agricultural landscapes. In: Crooks, K.R., Sanjayan, M. (Eds.), Connectivity Conservation. Cambridge University Press, Cambridge, pp. 255-289.

Rico-Gray, V., Oliveira, P.S., 2007. The Ecology and Evolution of Ant-Plant Interactions. University of Chicago Press, Chicago, IL.

Riegl, B., Luke, K.E., 1998. Ecological parameters of dynamited reefs in the northern Red Sea and their relevance to reef rehabilitation. Mar. Pollut. Bull. 37, 488-498.

Rietkerk, M., Dekker, S.C., de Ruiter, P.C., van de Koppel, J., 2004. Self-organized patchiness and catastrophic shifts in ecosystems. Science 305, 1926-1929.

Rodríguez-Cabal, M.A., Aizen, M.A., Novaro, A.J., 2007. Habitat fragmentation disrupts a plant-disperser mutualism in the temperate forest of South America. Biol. Conserv. 139, 195-202.

Rogers, L.E., Hinds, W.T., Buschbom, R.L., 1976. General weight vs. length relationship for insects. Ann. Entomol. Soc. Am. 69, 387-389.

Roland, J., Taylor, P.D., 1997. Insect parasitoid species respond to forest structure at different spatial scales. Nature 386, 710-713.

Rossberg, A.G., 2012. A complete analytic theory for structure and dynamics of populations and communities spanning wide ranges in body size. Adv. Ecol. Res. 46, 427-522.

Roubik, D.W., 1989. Ecology and Natural History of Tropical Bees. Cambridge University Press, Cambridge.

Roubik, D.W., 2006. Stingless bee nesting biology. Apidologie 37, 124-143.

Roy, D.B., Quinn, R.M., Gaston, K.J., 1998. Coincidence in the distribution of butterflies and their food plants. Ecography 21, 279-289.

Rundlöf, M., Smith, H.G., 2006. The effect of organic farming on butterfly diversity depends on landscape context. J. Appl. Ecol. 43, 1121-1127.

Rundlöf, M., Nilsson, H., Smith, H.G., 2008. Interacting effects of farming practice and landscape context on bumble-bees. Biol. Conserv. 141, 417-426.

Saavedra, S., Stouffer, D.B., Uzzi, B., Bascompte, J., 2011. Strong contributors to network persistence are the most vulnerable to extinction. Nature 478, 233-235.

Sabatino, M., Maceira, N., Aizen, M.A., 2010. Direct effects of habitat area on interaction diversity in pollination webs. Ecol. Appl. 20, 1491-1497.

Sæther, B.-E., Engen, S., Lande, R., 1999. Finite metapopulation models with density-dependent migration and stochastic local dynamics. Proc. R. Soc. B 266, 113-118. 
Santos, T., Telleria, J., Virgos, E., 1999. Dispersal of Spanish juniper Juniperus thurifera by birds and mammals in a fragmented landscape. Ecography 22, 193-204.

Santos, B.A., Peres, C.A., Oliveira, M.A., Grillo, A., Alves-Costa, C.P., Tabarelli, M., 2008. Drastic erosion in functional attributes of tree assemblages in Atlantic forest fragments of northeastern Brazil. Biol. Conserv. 141, 249-260.

Sargent, R.D., Kembel, S.W., Emery, N.C., Forrestel, E.J., Ackerly, D.D., 2011. Effect of local community phylogenetic structure on pollen limitation in an obligately insectpollinated plant. Am. J. Bot. 98, 283-289.

Saunders, D.A., Hobbs, R.J., Margules, C.R., 1991. Biological consequences of ecosystem fragmentation: a review. Conserv. Biol. 5, 18-32.

Schaffer, M.L., 1981. Minimum population sizes for species conservation. Bioscience 31, 131-134.

Schleuning, M., Blüthgen, N., Flörchinger, M., Braun, J., Schaefer, H.M., Böhning-Gaese, K., 2011a. Specialization and interaction strength in a tropical plantfrugivore network differ among forest strata. Ecology 92, 26-36.

Schleuning, M., Farwig, N., Peters, M.K., Bergsdorf, T., Bleher, B.R., Brandl, R., Dalitz, H., Fischer, G., Freund, W., Gikungu, M.W., Hagen, M., Garcia, F.H., et al., 2011b. Forest fragmentation and selective logging have inconsistent effects on multiple animal-mediated ecosystem processes in a tropical forest. PLoS One 6, e27785.

Schnitzler, F.-R., Hartley, S., Lester, P.J., 2011. Trophic-level responses differ at plant, plot and fragment levels in urban native forest fragments: a hierarchical analysis. Ecol. Entomol. 36, 241-250.

Schupp, E.W., Jordano, P., Gómez, J.M., 2010. Seed dispersal effectiveness revisited: a conceptual review. New Phytol. 188, 333-353.

Schurr, F.M., Spiegel, O., Steinitz, O., Trakhtenbrot, A., Tsoar, A., Nathan, R., 2009. Long-distance seed dispersal. Annu. Plant Rev. 38, 204-237.

Shanahan, M., Harrison, R.D., Yamuna, R., Boen, W., Thornton, I.W.B., 2001. Colonization of an island volcano, Long Island, Papua New Guinea, and an emergent island, Motmot, in its caldera lake. V. Colonization by figs (Ficus spp.), their dispersers and pollinators. J. Biogeogr. 28, 1365-1377.

Sieving, K.E., Karr, J.R., 1997. Avian extinction and persistence mechanisms in lowland Panama. In: Laurance, W.F., Bierregaard, R.O. (Eds.), Tropical Forest Remnants. University of Chicago Press, Chicago, IL, pp. 156-170.

Sih, A., Baltus, M.-S., 1987. Patch size, pollinator behavior, and pollinator limitation in catnip. Ecology 68, 1679-1690.

Silliman, B.R., van de Koppel, J., Bertness, M.D., Stanton, L., Mendelsohn, I., 2005. Drought, snails, and large-scale die-off of southern U.S. salt marshes. Science 310, 1803-1806.

Simberloff, D., Cox, J., 1987. Consequences and costs of conservation corridors. Conserv. Biol. 1, 63-71.

Simberloff, D., Farr, J.A., Cox, J., Mehlman, D.W., 1992. Movement corridors, conservation bargains or poor investments? Conserv. Biol. 6, 493-504.

Sinclair, A.R.E., Mduma, S., Brashares, J.S., 2003. Patterns of predation in a diverse predator-prey system. Nature 425, 288-290.

Sisk, T.D., Battin, J., 2002. Habitat edges and avian ecology: geographic patterns and insights for western landscapes. Stud. Avian Biol. 25, 30-48.

Solé, R.V., Montoya, J.M., 2006. Ecological network meltdown from habitat loss and fragmentation. In: Pascual, M., Dunne, J. (Eds.), Ecological Networks: Linking Structure to Dynamics in Food Webs. Oxford University Press, New York, pp. 305-323.

Spiegel, O., Nathan, R., 2007. Incorporating dispersal distance into the disperser effectiveness framework: frugivorous birds provide complementary dispersal to plants in a patchy environment. Ecol. Lett. 10, 718-728. 
Srivastava, D.S., 2006. Habitat structure, trophic structure and ecosystem function: interactive effects in a bromeliad-insect community. Oecologia 149, 493-504.

Stamps, J.A., Buechner, M., Krishnan, V.V., 1987a. The effects of edge permeability and habitat geometry on emigration from patches of habitat. Am. Nat. 129, 533-552.

Stamps, J.A., Buechner, M., Krishnan, V.V., 1987b. The effects of habitat geometry on territorial defense costs: intruder pressure in bounded habitats. Am. Zool. 27, 307-325.

Stang, M., Klinkhamer, P.G.L., van der Meijden, E., 2006. Size constraints and flower abundance determine the number of interactions in a plant-flower visitor web. Oikos 112, 111-121.

Stang, M., Klinkhamer, P.G.L., van der Meijden, E., 2007. Asymmetric specialization and extinction risk in plant-flower visitor webs: a matter of morphology or abundance? Oecologia 151, 442-453.

Stang, M., Klinkhamer, P.G.L., Waser, N.M., Stang, I., van der Meijden, E., 2009. Sizespecific interaction patterns and size matching in a plant-pollinator interaction web. Ann. Bot. 103, 1459-1469.

Steffan-Dewenter, I., 2003. Importance of habitat and landscape context for species richness of bees and wasps in fragmented orchard meadows. Conserv. Biol. 17, 1036-1044.

Steffan-Dewenter, I., Tscharntke, T., 1999. Effects of habitat isolation on pollinator communities and seed set. Oecologia 121, 432-440.

Steffan-Dewenter, I., Tscharntke, T., 2002. Insect communities and biotic interaction on fragmented calcareous grasslands: a mini review. Biol. Conserv. 104, 275-284.

Steffan-Dewenter, I., Munzenberg, U., Burger, C., Thies, C., Tscharntke, T., 2002. Scale-dependent effects of landscape context on three pollinator guilds. Ecology 83, 1421-1432.

Steffan-Dewenter, I., Klein, A.M., Gaebele, V., Alfert, T., Tscharntke, T., 2006. Bee diversity and plant-pollinator interactions in fragmented landscapes. In: Waser, N.M., Ollerton, J. (Eds.), Plant-Pollinator Interactions: From Specialization to Generalization. University of Chicago Press, Chicago, IL, pp. 387-407.

Stephens, S.E., Koons, D.N., Rotella, J.J., Willey, D.W., 2003. Effects of habitat fragmentation on avian nesting success: a review of the evidence at multiple spatial scales. Biol. Conserv. 115, 101-110.

Stevens, V.M., Leboulengé, È., Wesselingh, R.A., Baguette, M., 2006. Quantifying functional connectivity, experimental assessment of boundary permeability for the natterjack toad (Bufo calamita). Oecologia 150, 161-171.

Stouffer, D.B., Bascompte, J., 2010. Understanding food-web persistence from local to global scales. Ecol. Lett. 13, 154-161.

Strickler, K.L., 1979. Specialization and foraging efficiency of solitary bees. Ecology 60, 998-1009.

Sutherland, G.D., Harestad, A.S., Price, K., Lertzman, K.P., 2000. Scaling of natal dispersal distances in terrestrial birds and mammals. Conserv. Ecol. 4, 16.

Syms, C., Jones, G.P., 2000. Disturbance, habitat structure, and the dynamics of a coral-reef fish community. Ecology 81, 2714-2729.

Tabarelli, M., Peres, C.A., 2002. Abiotic and vertebrate seed dispersal in the Brazilian Atlantic forest: implications for forest regeneration. Biol. Conserv. 106, 165-176.

Tabarelli, M., Mantovani, W., Peres, C.A., 1999. Effects of habitat fragmentation on plant guild structure in the montane Atlantic forest of southeastern Brazil. Biol. Conserv. 91, 119-127.

Tabarelli, M., Lopes, A.V., Peres, C.A., 2008. Edge-effects drive tropical forest fragments towards an early-successional system. Biotropica 40, 657-661.

Tabarelli, M., Aguiar, A.V., Girão, L.C., Peres, C.A., Lopes, A.V., 2010. Effects of pioneer tree species hyperabundance on forest fragments in Northeastern Brazil. Conserv. Biol. 24, 1654-1663. 
Taylor, P.D., Fahrig, L., Henein, K., Merriam, G., 1993. Connectivity is a vital element of landscape structure. Oikos 68, 571-573.

Taylor, P.D., Fahrig, L., With, K.A., 2006. Landscape connectivity: a return to the basics. In: Crooks, K.R., Sanjayan, M. (Eds.), Connectivity Conservation. Cambridge University Press, New York, pp. 29-43.

Terborgh, J., Lopez, L., Tello, J., Yu, D., Bruni, A.R., 1997. Transitory states in relaxing ecosystems of land bridge islands. In: Laurance, W.F., Bierregaard, R.O. (Eds.), Tropical Forest Remnants: Ecology, Management, and Conservation of Fragmented Communities. University of Chicago Press, Chicago, IL, pp. 256-274.

Terborgh, J., Lopez, L., Nuñez, P., Rao, M., Shahabuddin, G., Orihuela, G., Riveros, M., Ascanio, R., Adler, G.H., Lambert, T.D., Balbas, L., 2001. Ecological meltdown in predator-free forest fragments. Science 294, 1923-1926.

Tewksbury, J.J., Levey, D.J., Haddad, N.M., Sargent, S., Orrock, J.L., Weldon, A., Danielson, B.J., Brinkerhoff, J., Damschen, E.I., Townsend, P., 2002. Corridors affect plants, animals, and their interactions in fragmented landscapes. Proc. Natl. Acad. Sci. USA 99, 12923-12926.

Thebault, E., Fontaine, C., 2010. Stability of ecological communities and the architecture of mutualistic and trophic networks. Science 329, 853-856.

Thies, C., Roschewitz, I., Tscharntke, T., 2005. The landscape context of cereal aphidparasitoid interactions. Proc. R. Soc. Lond. B 272, 203-210.

Thomas, C.D., 2000. Dispersal and extinction in fragmented landscapes. Proc. R. Soc. Lond. B 267, 139-145.

Thompson, J.N., 2005. The Geographic Mosaic of Coevolution. University of Chicago Press, Chicago, IL.

Tilman, D., May, R.M., Lehman, C.L., Nowak, M.A., 1994. Habitat destruction and the extinction debt. Nature 371, 65-66.

Tilman, D., Fargione, J., Wolff, B., D’Antonio, C., Dobson, A., Howarth, R., Schindler, D., Schlesinger, W.H., Simberloff, D., Swackhamer, D., 2001. Forecasting agriculturally driven global environmental change. Science 292, 281-284.

Tischendorf, L., Fahrig, L., 2000. On the usage and measurement of landscape connectivity. Oikos 90, 7-19.

Torp, E., 1994. Danmarks Svirrefluer, vol. 6. Apollo, Stenstrup, Denmark.

Townsend, C.R., Hildrew, A.G., Francis, J.E., 1983. Community structure in some southern English streams: the influence of physiochemical factors. Freshw. Biol. 19, 521-544.

Townsend, C.R., Hildrew, A.G., Schofield, K., 1987. Persistence of stream invertebrate communities in relation to environmental variability. J. Anim. Ecol. 56, 597-613.

Trøjelsgaard, K., Olesen, J.M., 2012. Macroecology of pollination networks. Global Ecol. Biogeogr http://dx.doi.org/10.1111/j.1466-8238.2012.00777.x.

Tscharntke, T., Steffan-Dewenter, I., Kruess, A., Thies, C., 2002. Characteristics of insect populations on habitat fragments: a mini review. Ecol. Res. 17, 229-239.

Tscharntke, T., Klein, A.M., Kruess, A., Steffan-Dewenter, I., Thies, C., 2005. Landscape perspectives on agricultural intensification and biodiversity-ecosystem service management. Ecol. Lett. 8, 857-874.

Turing, A.M., 1952. The chemical basis of morphogenesis. Proc. R. Soc. Lond. B 237, $37-72$.

Tylianakis, J.M., Tscharntke, T., Lewis, O.T., 2007. Habitat modification alters the structure of tropical host-parasitoid food webs. Nature 445, 202-205.

Tylianakis, J.M., Didham, R.K., Bascompte, J., Wardle, D.A., 2008. Global change and species interactions in terrestrial ecosystems. Ecol. Lett. 11, 1351-1363.

Tylianakis, J.M., Laliberté, E., Nielsen, A., Bascompte, J., 2010. Conservation of species interaction networks. Biol. Conserv. 143, 2270-2279. 
Urban, D.L., Keitt, T., 2001. Landscape connectivity: a graph-theoretic perspective. Ecology 82, 1205-1218.

Urban, D.L., O’Neill, R.V., Shugart, H.H., 1987. Landscape ecology: a hierarchical perspective can help scientists understand spatial patterns. Bioscience 37, 119-127.

Urban, D.L., Minor, E.S., Treml, E.A., Schick, R.S., 2009. Graph models of habitat mosaics. Ecol. Lett. 12, 260-273.

Uriarte, M., Anciaes, M., Da Silva, M.T.B., Rubim, P., Johnson, E., Bruna, E.M., 2011. Disentangling the drivers of reduced long-distance seed dispersal by birds in an experimentally fragmented landscape. Ecology 92, 924-937.

Valladares, G., Cagnolo, L., Salvo, A., 2012. Forest fragmentation leads to food web contraction. Oikos 121, 299-305.

Vamosi, J.C., Knight, T.M., Steets, J.A., Mazer, S.J., Burd, M., Ashman, T.-L., 2006. Pollination decays in biodiversity hotspots. Proc. Natl. Acad. Sci. USA 103, 956-961.

van Houtan, K.S., Pimm, S.L., Halley, J.M., Bierregaard, R.O., Lovejoy, T.E., 2007. Dispersal of Amazonian birds in continuous and fragmented forest. Ecol. Lett. 10, 219-229.

van Nieuwstadt, M.G.L., Ruano Iraheta, C.E., 1996. Relation between size and foraging range in stingless bees (Apidae, Meliponinae). Apidologie 27, 219-228.

van Nouhuys, S., 2005. Effects of habitat fragmentation at different trophic levels in insect communities. Ann. Zool. Fennici 42, 433-447.

van Nouhuys, S., Hanski, I., 2002. Colonization rates and distances of a host butterfly and two specific parasitoids in a fragmented landscape. J. Anim. Ecol. 71, 639-650.

Vanbergen, A.J., Hails, R.S., Watt, A.D., Jones, T.H., 2006. Consequences for host-parasitoid interactions of grazing-dependent habitat heterogeneity. J. Anim. Ecol. 75, 789-801.

Vanbergen, A.J., Jone, T.H., Hail, R.S., Watt, A.D., Elston, D.A., 2007. Consequences for a host-parasitoid interaction of host-plant aggregation, isolation and phenology. Ecol. Entomol. 32, 419-427.

Vandermeer, J., Perfecto, I., Philpott, S.M., 2010. Ecological complexity and pest control in organic coffee production: uncovering an autonomous ecosystem service. Bioscience 60, $527-537$.

Vázquez, D.P., 2005. Degree distribution in plant-animal mutualistic networks: forbidden links or random interactions? Oikos 108, 421-426.

Vázquez, D.P., Morris, W.F., Jordano, P., 2005. Interaction frequency as a surrogate for the total effect of animal mutualists on plants. Ecol. Lett. 8, 1088-1094.

Vázquez, D.P., Melián, C.J., Williams, N.M., Blüthgen, N., Krasnov, B.R., Poulin, R., 2007. Species abundance and asymmetric interaction strength in ecological networks. Oikos 116, 1120-1127.

Vázquez, D.P., Chacoff, N.P., Cagnolo, L., 2009. Evaluating multiple determinants of the structure of plant-animal mutualistic networks. Ecology 90, 2039-2046.

Verdú, M., Valiente-Banuet, A., 2011. The relative contribution of abundance and phylogeny to the structure of plant facilitation networks. Oikos 120, 1351-1356.

Voigt, F.A., Bleher, B., Fietz, J., Ganzhorn, J.U., Schwab, D., Böhning-Gaese, K., 2004. A comparison of morphological and chemical fruit traits between two sites with different frugivore assemblages. Oecologia 141, 94-104.

Vörösmarty, C.J., McIntyre, P.B., Gessner, M.O., Dudgeon, D., Prusevich, A., Green, P., Glidden, S., Bunn, S.E., Sullivan, C.A., Liermann, C.R., Davies, P.M., 2010. Global threats to human water security and river biodiversity. Nature 467, 555-561.

Walker, D.I., McComb, A.J., 1992. Seagrass degradation in Australian coastal waters. Mar. Pollut. Bull. 25, 191-195.

Walker, D.I., Kendrick, G.A., McComb, A.J., 2006. Decline and recovery of seagrass ecosystems: the dynamics of change. In: Larkum, A.W.D., Orth, R.J., Duarte, C.M. (Eds.), Seagrasses: Biology, Ecology and Conservation. Springer-Verlag, Dordrecht, pp. 551-565. 
Warren, P.H., 1996. Dispersal and destruction in a multiple habitat system: an experimental approach using protist communities. Oikos 77, 317-325.

Weibull, A.C., Bengtsson, J., Nohlgren, E., 2000. Diversity of butterflies in the agricultural landscape: the role of farming system and landscape heterogeneity. Ecography 24, 743-750.

Wells, S., 2009. Dynamite fishing in northern Tanzania-pervasive, problematic and yet preventable. Mar. Pollut. Bull. 58, 20-23.

Whittaker, R.J., Triantis, K.A., Ladle, R.J., 2008. A general dynamic theory of oceanic island biogeography. J. Biogeogr. 35, 977-994.

Wikelski, M., Moxley, J., Eaton-Mordas, A., López-Uribe, M.M., Holland, R., Moskowitz, D., Roubik, D.W., Kays, R., 2010. Large-range movements of neotropical orchid bees observed via radio telemetry. PLoS One 5, e10738.

Wilcock, H.R., Nichols, R.A., Hildrew, A.G., 2003. Genetic population structure and neighbourhood population size estimates of the caddisfly Plectrocnemia conspersa. Freshw. Biol. 48, 1813-1824.

Wilcove, D.S., McLellan, C.H., Dobson, A.P., 1986. Habitat fragmentation in the temperate zone. In: Soulé, M.E. (Ed.), Conservation Biology: The Science of Scarcity and Diversity. Sinauer Associates, Sunderland, MA, pp. 237-256.

Willems, J.H., Huijsmans, K.G.A., 1994. Vertical seed dispersal by earthworms: a quantitative approach. Ecography 17, 124-130.

Willi, Y., van Buskirk, J., Fischer, M., 2005. A threefold genetic allee effect: population size affects cross-compatibility, inbreeding depression and drift load in the self-incompatible Ranunculus reptans. Genetics 169, 2255-2265.

Williams, P.H., 2005. Does specialization explain rarity and decline among British bumblebees? a response to Goulson et al. Biol. Conserv. 122, 33-43.

Williams, R.J., Martinez, N.D., 2000. Simple rules yield complex food webs. Nature 404, 180-183.

Williams, P.H., Colla, S., Xie, Z., 2009. Bumblebee vulnerability: common correlates of winners and losers across three continents. Conserv. Biol. 23, 931-940.

Willis, E.O., 1979. The composition of avian communities in remanescent wood lots in southeastern Brazil. Pap. Avul. Zool. 33, 1-25.

Willson, M.F., Whelan, C.J., 1990. The evolution of fruit color in fleshy-fruited plants. Am. Nat. 136, 790-809.

Wirth, R., Meyer, S.T., Leal, I.R., Tabarelli, M., 2008. Plant-herbivore interactions at the forest edge. Progr. Bot. 69, 423-448 1.

Woodward, G., Hildrew, A.G., 2002a. Food web structure in riverine landscapes. Freshw. Biol. 47, 777-798.

Woodward, G., Hildrew, A.G., 2002b. Differential vulnerability of prey to an invading top predator: integrating field surveys and laboratory experiments. Ecol. Entomol. 27, 732-744.

Woodward, G., Ebenman, B., Emmerson, M., Montoya, J.M., Olesen, J.M., Valido, A., Warren, P.H., 2005. Body size in ecological networks. Trends Ecol. Evol. 20, 402-409.

Woodward, G., Papantoniou, G., Edwards, F., Lavridsen, R.B., 2008. Trophic trickles and cascades in a complex food web: impacts of a keystone predator on stream community structure and ecosystem processes. Oikos 117, 683-692.

Woodward, G., Benstead, J.P., Beveridge, O.S., Blanchard, J., Brey, T., Brown, L.E., Cross, W.F., Friberg, N., Ings, T.C., Jacob, U., Jennings, S., Ledger, M.E., et al., 2010a. Ecological networks in a changing climate. Adv. Ecol. Res. 42, 71-138.

Woodward, G., Blanchard, J., Lauridsen, R.B., Edwards, F.K., Jones, J.I., Figueroa, D., Warren, P.H., Petchey, O.L., 2010b. Individual-based food webs: species identity, body size and sampling effects. Adv. Ecol. Res. 43, 211-266. 
Woodward, G., Brown, L.E., Edwards, F.K., Hudson, L.N., Milner, A.M., Reuman, D.C., Ledger, M.E., 2012. Climate change impacts in multispecies systems: drought alters food web size-structure in a field experiment. Philos. Trans. R. Soc. Lond. B in press 87, $526-544$.

Wright, S.J., Duber, H.C., 2001. Poachers and forest fragmentation alter seed dispersal, seed survival, and seedling recruitment in the palm Attalea butyraceae, with implications for tropical tree diversity. Biotropica 33, 583-595.

Young, A., Boyle, T., Brown, T., 1996. The population genetic consequences of habitat fragmentation for plants. Trends Ecol. Evol. 11, 413-418.

Yu, L., Lu, J., 2011. Does landscape fragmentation influence sex ratio of dioecious plants? A case study of Pistacia chinensis in the Thousand-Island Lake region of China. PLoS One 6, e22903.

Zamora, R., 2000. Functional equivalence in plant-animal interactions: ecological and evolutionary consequences. Oikos 88, 442-447.

Zhang, F., Hui, C., Terblanche, J.S., 2011. An interaction switch predicts the nested architecture of mutualistic networks. Ecol. Lett. 14, 797-803. 